\title{
Reliability-Based Condition
}

Assessment of Steel

Containment and Liners

\author{
RECEIVED \\ DEC 171996 \\ OSTI
}

Prepared by

B. Ellingwood, B. Bhattacharya, R. Zheng, JHU

The Johns Hopkins University

Oak Ridge National Laboratory

Prepared for

U.S. Nuclear Regulatory Commission 


\section{AVAILABILITY NOTICE \\ Availability of Reference Materials Cited in NRC Publications}

Most documents cited in NRC publications will be available from one of the following sources:

1. The NRC Public Document Room, 2120 L Street, NW., Lower Level, Washington, DC 20555-0001

2. The Superintendent of Documents, U.S. Government Printing Office, P. O. Box 37082, Washington, DC 20402-9328

3. The National Technical Information Service, Springfield, VA 22161-0002

Although the listing that follows represents the majority of documents cited in NRC publications, it is not intended to be exhaustive.

Referenced documents available for inspection and copying for a fee from the NRC Public Document Room include NRC correspondence and internal NRC memoranda: NRC bulletins, circulars, information notices, inspection and investigation notices; licensee event reports; vendor reports and correspondence; Commission papers: and applicant and licensee documents and correspondence.

The following documents in the NUREG series are available for purchase from the Government Printing Office: formal NRC staff and contractor reports, NRC-sponsored conference proceedings, international agreement reports, grantee reports, and NRC booklets and brochures. Also available are regulatory guides, NRC regulatlons in the Code of Federal Regulations, and Nuclear Regulatory Commission Issuances.

Documents avallable from the Natlonal Technical Information Service include NUREG-series reports and technical reports prepared by other Federal agencies and reports prepared by the Atomic Energy Commission, forerunner agency to the Nuclear Regulatory Commission.

Documents available from public and special technical libraries include all open literature items, such as books, journal articles, and transactions. Federal Register notices. Federal and State legislation, and congressional reports can usually be obtained from these libraries.

Documents such as theses, dissertations, foreign reports and translations, and non-NRC conference proceedings are available for purchase from the organization sponsoring the publication cited.

Single coples of NRC draft reports are available free. to the extent of supply, upon written request to the Office of Administration, Distribution and Mail Services Section. U.S. Nuclear Regulatory Commission, Washington, DC 20555-0001.

Coples of industry codes and standards used in a substantive manner in the NRC regulatory process are maintained at the NRC Library. Two White Flint North. 11545 Rockville Pike, Rockville. MD 20852-2738, for use by the public. Codes and standards are usually copyrighted and may be purchased from the originating organization or, if they are American National Standards. from the American National Standards Institute. 1430 Broadway, New York, NY 10018-3308.

\section{DISCLAIMER NOTICE}

This report was prepared as an account of work sponsored by an agency of the United States Govemment. Neither the United States Govemment nor any agency thereof, nor any of their employees, makes any warranty, expressed or implied, or assumes any legal liability or responsibility for any third party's use, or the results of such use, of any information, apparatus, product, or process disclosed in this report, or represents that its use by such third party would not infringe privately owned rights. 


\section{Reliability-Based Condition Assessment of Steel Containment and Liners}

Manuscript Completed: October 1996

Date Published: November 1996

Prepared by

B. Ellingwood, B. Bhattacharya, R. Zheng, JHU

Oak Ridge National Laboratory

Managed by Lockheed Martin Energy Research Corporation

Oak Ridge, TN 37831

Subcontractor

The Johns Hopkins University

Department of Civil Engineering

Baltimore, MD 21218

W. E. Norris, NRC Project Manager

DISTRIBUTION OF THIS DOCUMENT IS UALLHATED

Prepared for

Division of Engineering Technology

Office of Nuclear Regulatory Research

U.S. Nuclear Regulatory Commission

Washington, DC 20555-0001

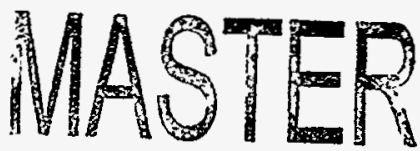

NRC Job Code J6043 


\section{DISCLAIMER}

This report was prepared as an account of work sponsored by an agency of the United States Government. Neither the United States Government nor any agency thereof, nor any of their employees, makes any warranty, express or implied, or assumes any legal liability or responsibility for the accuracy, completeness, or usefulness of any information, apparatus, product, or process disclosed, or represents that its use would not infringe privately owned rights. Reference berein to any specific commercial product, process, or service by trade name, trademark, manufacturer, or otherwise does not necessarily constitute or imply its endorsement, recommendation, or favoring by the United States Government or any agency thereof. The views and opinions of authors expressed herein do not necessarily state or reflect those of the United States Government or any agency thereof. 


\section{DISCLAIMER}

Portions of this document may be illegible in electronic image products. Images are produced from the best available original document. 


\begin{abstract}
Steel containments and liners in nuclear power plants may be exposed to aggressive environments that may cause their strength and stiffness to decrease during the plant service life. Among the factors recognized as having the potential to cause structural deterioration are uniform, pitting or crevice corrosion; fatigue, including crack initiation and propagation to fracture; elevated temperature; and irradiation. The evaluation of steel containments and liners for continued service must provide assurance that they are able to withstand future extreme loads during the service period with a level of reliability that is sufficient for public safety. Rational methodologies to provide such assurances can be developed using modern structural reliability analysis principles that take uncertainties in loading, strength, and degradation resulting from environmental factors into account.

The research described in this report is in support of the Steel Containments and Liners Program being conducted for the U.S. Nuclear Regulatory Commission by the Oak Ridge National Laboratory. The research demonstrates the feasibility of using reliability analysis as a tool for performing condition assessments and service life predictions of steel containments and liners. Mathematical models that describe time-dependent changes in steel due to aggressive environmental factors are identified, and statistical data supporting the use of these models in time-dependent reliability analysis are summarized. The analysis of steel containment fragility is described, and simple illustrations of the impact on reliability of structural degradation are provided. The role of nondestructive evaluation in time-dependent reliability analysis, both in terms of defect detection and sizing, is examined. A Markov model provides a tool for accounting for time-dependent changes in damage condition of a structural component or system.
\end{abstract}





\section{TABLE OF CONTENTS}

Page

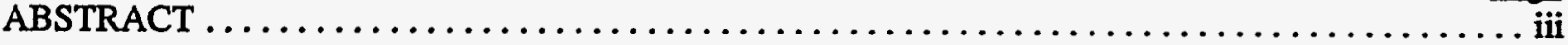

TABLE OF CONTENTS $\ldots \ldots \ldots \ldots \ldots \ldots \ldots \ldots \ldots \ldots \ldots \ldots \ldots \ldots \ldots \ldots \ldots \ldots, \mathrm{v}$

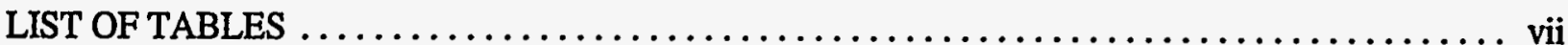

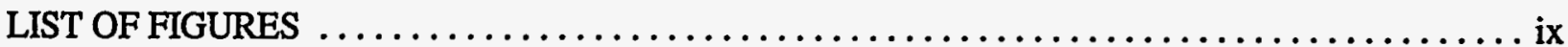

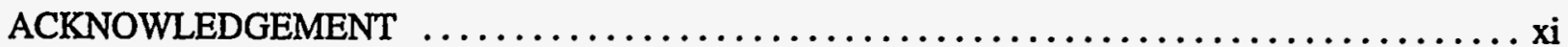

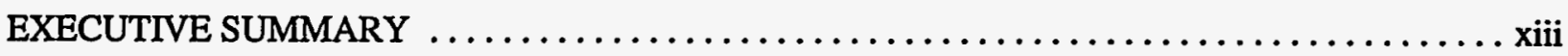

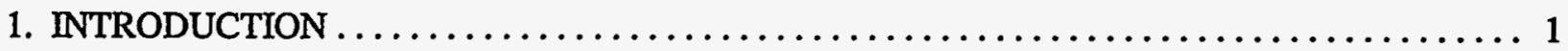

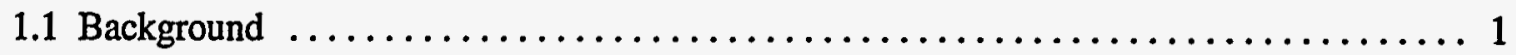

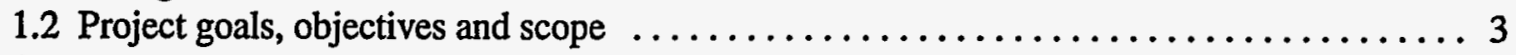

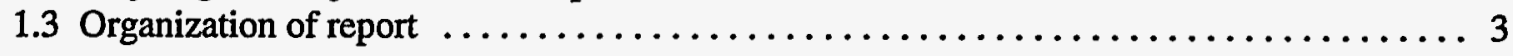

2. STRUCTURAL DETERIORATION AND ITS EVALUATION $\ldots \ldots \ldots \ldots \ldots \ldots \ldots \ldots, 5$

2.1 Corrosion. ............................................ 6

2.1.1 General or uniform corrosion. ............................... 6

2.1.2 Localized corrosion - pitting and crevice...................... 8

2.1.3 Deterioration of coatings. ................................ 9

2.2 Fatigue and fracture $\ldots \ldots \ldots \ldots \ldots \ldots \ldots \ldots \ldots \ldots \ldots \ldots \ldots \ldots \ldots \ldots \ldots \ldots \ldots, 10$

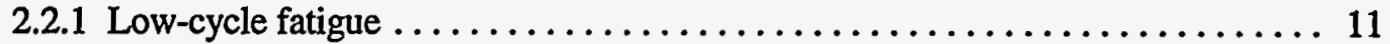

2.2.2 Crack propagation and fracture $\ldots \ldots \ldots \ldots \ldots \ldots \ldots \ldots \ldots \ldots \ldots \ldots \ldots \ldots$

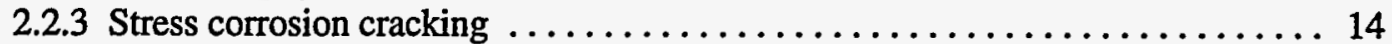

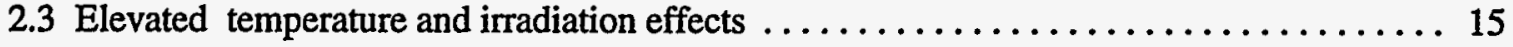

2.4 Summary $\ldots \ldots \ldots \ldots \ldots \ldots \ldots \ldots \ldots \ldots \ldots \ldots \ldots \ldots \ldots \ldots \ldots \ldots \ldots \ldots, 15$

3. NONDESTRUCTIVE EVALUATION METHODS $\ldots \ldots \ldots \ldots \ldots \ldots \ldots \ldots \ldots \ldots \ldots$

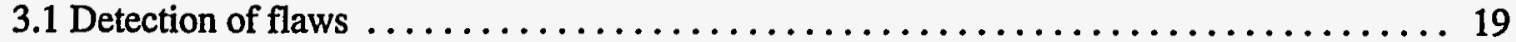

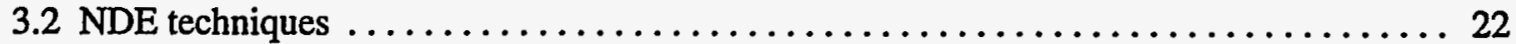

3.2.1 Surface and near-surface methods $\ldots \ldots \ldots \ldots \ldots \ldots \ldots \ldots \ldots \ldots \ldots, 22$

3.2 .2 Ultrasonic inspection (UT) $\ldots \ldots \ldots \ldots \ldots \ldots \ldots \ldots \ldots \ldots \ldots \ldots \ldots \ldots \ldots \ldots \ldots, 23$

3.2 .3 Eddy current $(\mathrm{EC}) \quad \ldots \ldots \ldots \ldots \ldots \ldots \ldots \ldots \ldots \ldots \ldots \ldots \ldots \ldots \ldots \ldots \ldots \ldots, 23$

3.2 .4 Acoustic emission (AE) $\ldots \ldots \ldots \ldots \ldots \ldots \ldots \ldots \ldots \ldots \ldots \ldots \ldots \ldots, 24$

3.2.5 Radiography (RT) $\ldots \ldots \ldots \ldots \ldots \ldots \ldots \ldots \ldots \ldots \ldots \ldots \ldots \ldots \ldots, 24$

3.3 Flaw measurement errors $\ldots \ldots \ldots \ldots \ldots \ldots \ldots \ldots \ldots \ldots \ldots \ldots \ldots \ldots \ldots \ldots, 24$

3.4 Summary $\ldots \ldots \ldots \ldots \ldots \ldots \ldots \ldots \ldots \ldots \ldots \ldots \ldots \ldots \ldots \ldots \ldots \ldots \ldots \ldots \ldots, 26$

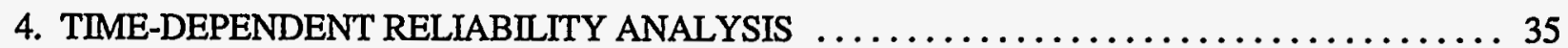

4.1 Probabilistic models of loads $\ldots \ldots \ldots, \ldots, \ldots, \ldots, \ldots, \ldots, \ldots, \ldots, \ldots, \ldots, 35$

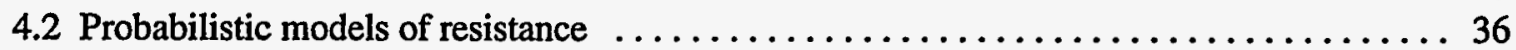

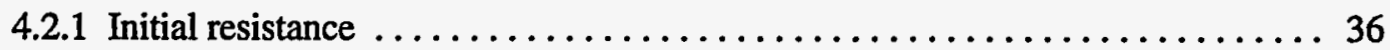

4.2.2 Time-dependent deterioration in resistance $\ldots \ldots \ldots \ldots \ldots \ldots \ldots \ldots \ldots, 37$

4.2.3 Fragility modeling of steel containments and liners $\ldots \ldots \ldots \ldots \ldots \ldots \ldots, 38$ 
4.3 Time-dependent reliability analysis of degrading structures $\ldots \ldots \ldots \ldots \ldots \ldots \ldots \ldots \ldots 1$

4.3.1 Degradation independent of service loads ................... 42

4.3.2 Illustration of time-dependent reliability - corrosion $\ldots \ldots \ldots \ldots \ldots \ldots \ldots . \ldots 4$

4.3.3 Degradation dependent on service loads $\ldots \ldots \ldots \ldots \ldots \ldots \ldots \ldots \ldots 45$

4.3.4 Illustration of time-dependent reliability - fatigue $\ldots \ldots \ldots \ldots \ldots \ldots \ldots . .48$

4.3.5 Reliability of Structural Systems $\ldots \ldots \ldots \ldots \ldots \ldots \ldots \ldots \ldots \ldots \ldots . \ldots . \ldots . \ldots . \ldots . \ldots$

4.3.6 Appraisal of Structural Reliability Methods $\ldots \ldots \ldots \ldots \ldots \ldots \ldots \ldots \ldots$

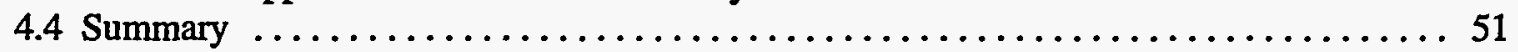

5. TECHNIQUES FOR IN-SERVICE RISK MANAGEMENT $\ldots \ldots \ldots \ldots \ldots \ldots \ldots \ldots \ldots \ldots$

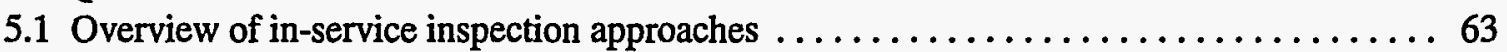

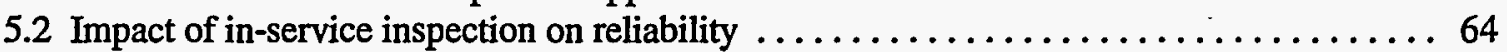

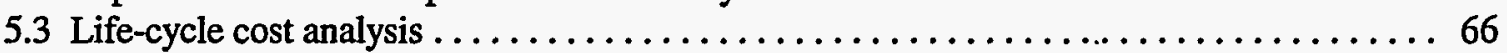

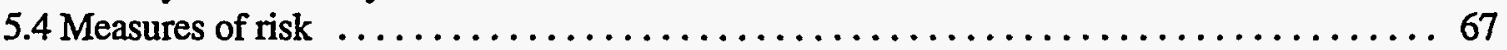

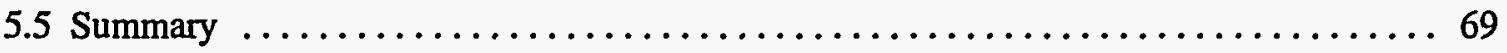

6. MARKOV PROCESS MODEL OF DAMAGE ACCUMULATION $\ldots \ldots \ldots \ldots \ldots \ldots \ldots \ldots$

7. RECOMMENDATIONS FOR FURTHER WORK $\ldots \ldots \ldots \ldots \ldots \ldots \ldots \ldots \ldots \ldots \ldots \ldots$

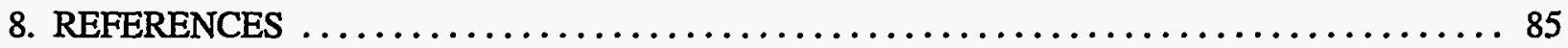




\section{LIST OF TABLES}

Page

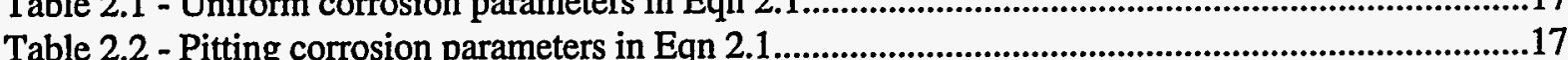

Table 4.1 - Summary of structural loads on NPPs.................................................................................52

Table 4.2 - Initial resistance of steel shapes and plates.................................................................................53 


\section{LIST OF FIGURES}

Figure 2.1 Typical S-N curves for fatigue design $\ldots . \ldots \ldots$

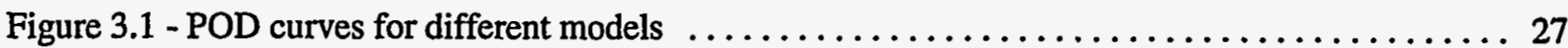

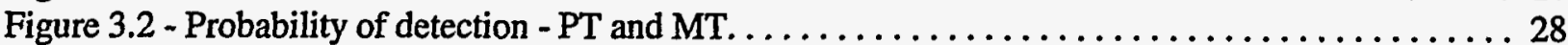

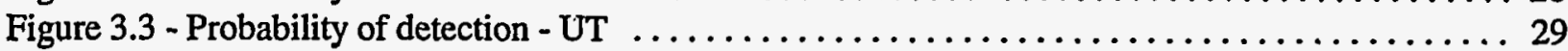

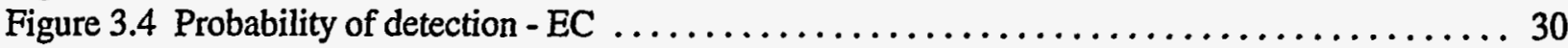

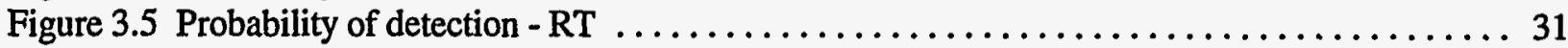

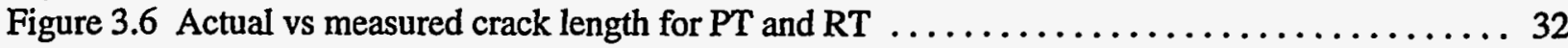

Figure 3.7 Actual vs measured crack depth including resolution limit $\ldots \ldots \ldots \ldots \ldots \ldots \ldots \ldots 33$

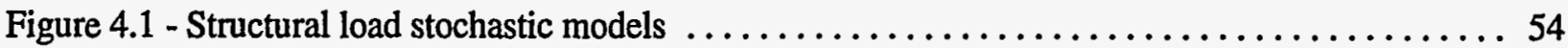

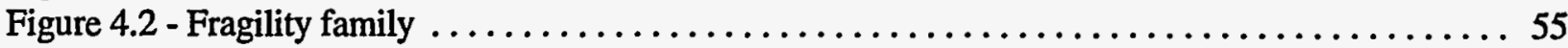

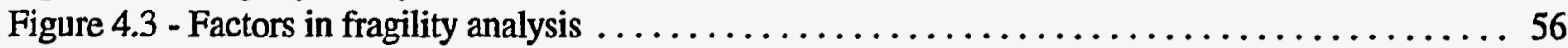

Figure 4.4 - Sample functions representing structural loads and degrading resistance $\ldots \ldots \ldots \ldots \ldots 57$

Figure 4.5 - Time-dependent reliability in tension $\left(D+P_{a}\right): \lambda=0.0017 / y r \ldots \ldots \ldots \ldots \ldots \ldots \ldots 58$

Figure 4.6 - Time-dependent reliability in tension $\left(\mathrm{D}+\mathrm{P}_{\mathrm{a}}\right) ; \lambda=0.001 / \mathrm{yr} \ldots \ldots \ldots \ldots \ldots \ldots \ldots \ldots . \ldots \ldots$

Figure 4.7 - Time-dependent reliability, with and without induction period for corrosion ........60

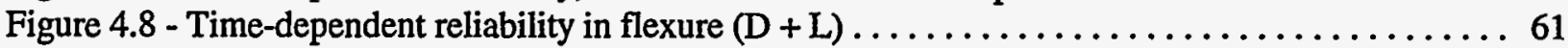

Figure 4.9 - Time-dependent reliability in flexure, with and without induction period for corrosion ...6 62

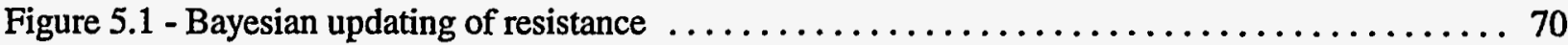

Figure 5.2 - Effect of in-service inspection and maintenance on $h(t) \ldots \ldots \ldots \ldots \ldots \ldots \ldots \ldots \ldots \ldots \ldots$

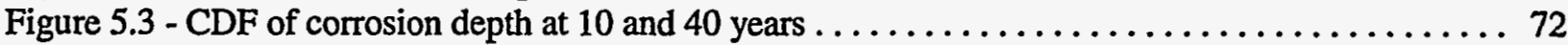

Figure $5.4-\mathrm{CDF}$ of corrosion depth, updated following inspection $\ldots \ldots \ldots \ldots \ldots \ldots \ldots \ldots \ldots$

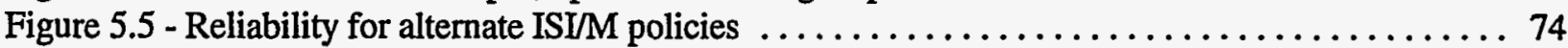




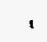

$\ldots$ 


\section{ACKNOWLEDGEMENT}

The authors would like to acknowledge the advice throughout the research that has been provided by Dr. Dan J. Naus of the Oak Ridge National Laboratory, and the financial assistance through Lockheed-Martin Energy Research Corp. Contract No. 19X-SP638V. Appreciation also is extended to Mr. Wallace E. Norris of the Division of Engineering Technology, U.S. Nuclear Regulatory Commission, and to Mr. C. Barry Oland of ORNL for helpful comments. The authors take full responsibility for the views expressed in this report. 


\section{EXECUTIVE SUMMARY}

Steel containments and liners in nuclear power plants (NPPs) may be exposed to aggressive environmental effects that may cause their strength and stiffness to decrease during the plant service life. Among the effects recognized as having the potential to cause structural deterioration are uniform, pitting or crevice corrosion; fatigue, including crack initiation and propagation to fracture; and elevated temperatures and irradiation. Such structural aging effects are well-recognized, at least qualitatively, in civil construction: bridges and highways, offshore structures, navigation infrastructure, and power plants. Although quantitative evaluation of aging effects on structural behavior is possible in some areas, it remains novel in most others. In particular, the evaluation of steel containments and liners in NPPs for continued service must provide assurance that they are able to withstand future extreme loads during a service period with an acceptable level of reliability. Rational methodologies to provide this assurance can be developed using modern structural reliability analysis principles that take uncertainties in loading, strength and degradation resulting from the above environmental effects into account.

The research described in this report supports the Steel Containments and Liners Program being conducted for the U.S. Nuclear Regulatory Commission by Oak Ridge National Laboratory. The goals of the research are to identify mathematical models from principles of mechanics to evaluate structural degradation; to recommend statistically-based sampling plans for nondestructive evaluation (NDE) of complex structures; and to identify methods to assess the probability that containment or liner capacity has not degraded, or will not degrade during a future service period. Section 2 reviews pertinent degradation mechanisms and associated statistical data, and proposes analytical methods for their treatment in condition assessment. Section 3 identifies common NDE techniques, with specific regard to their usefulness in time-dependent reliability analysis, flaw detection and measurement. Section 4 develops fundamental probabilistic methods for analyzing time-dependent reliability of steel containments and liners, emphasizing corrosion and fatigue effects, and illustrates their application for simple idealized structures. Section 5 discusses the role of in-service inspection, $\mathrm{NDE}$ and maintenance in reliability assurance and risk management. Section 6 presents a Markov model for tracking the evolution of damage in a structure throughout its service life, making provision for the role of periodic in-service inspection and maintenance on time-dependent reliability. Section 7 presents recommendations for further work. A comprehensive bibliography on time-dependent relaiblity analysis, with particular emphasis on reliability under conditions of corrosion and/or fatigue, concludes the report.

The first phase of this research has demonstrated the feasibility of using reliability analysis as a tool for performing condition assessments, evaluations of existing margins of safety, and service life predictions of steel containments and liners. Supporting statistical data and a demonstration of the application of the methodology to more complex structures are planned for the next phase of the research. 


\section{INTRODUCTION}

\subsection{Background}

Structural components and systems age during their service lives due to naturally occurring changes in material characteristics that may be initiated or accelerated by a particular service environment or extreme environmental conditions. Some of these changes have a relatively benign impact on structural strength or stiffness, while others may cause structural integrity to degrade over time. The potential for such changes to increase the hazard to public health and safety must be considered when evaluating an existing structure for continued service, particularly when the performance requirements may be different from those for which it was originally designed. Structural aging is a phenomenon that is well recognized, at least qualitatively, in civil construction: in bridges and highways, offshore structures, navigation infrastructure, and power plants. Quantitative evaluation of the structural impact of aging is possible in some areas but remains novel in most others. Research on structural aging is required to enhance or develop quantitative technical bases to support decisions regarding service life extensions.

Nuclear power plants (NPPs) have been operated safely in the United States according to regulations in Part 50 of Title 10 ("Energy") of the Code of Federal Regulations for many years, some for more than two decades. If these older plants were to be removed from service due to perceived structural aging effects, many utilities would suffer severe financial losses from decommissioning costs and the need to replace lost electric generating capacity. Many thermal or hydroelectric power plants continue to operate safely and economically for periods well in excess of their original design life. The design and operation of NPPs is highly regulated, and their safety record is exemplary, suggesting that service life extensions might be contemplated for nuclear plants as well.

Issues of managing aging in NPPs, evaluating service life extension, and associated safety issues have been a major research focus of the U.S. Nuclear Regulatory Commission for several years (Vora, et al, 1991; Shah and McDonald, 1989; Shah, et al, 1994). The research generally is following a five-step approach: (1) Identify and prioritize major components; (2) Identify degradation sites; (3) Assess advanced inspection/monitoring techniques; (4) Develop aging management approaches; and (5) Support development of a technical basis for aging management. To date, the focus of the program has been on replaceable mechanical and electrical components, for which aging issues often are believed to be most significant. The recently completed Structural Aging Program (Naus, et al, 1993; 1996) provides a methodology for condition assessment and reliability-based life prediction for concrete structures in NPPs. Little work has been done to date on the impact of aging on steel structures in NPPs.

Steel structures in NPPs are designed and constructed to withstand numerous operating and extreme environmental conditions and design-basis accident events (Standard Review Plan, 1981). Although major mechanical and electrical equipment items in a nuclear plant usually can be replaced, replacement or major repairs of the containment or other major steel structures are economically unfeasible. Evidence to support any proposed service life extension for a NPP must show that the capacity of the containment, containment liner and other safety-related steel structures in the plant to withstand extreme events has not deteriorated due to aging to the point where public health and safety are endangered. Current requirements for condition assessment and continued service evaluations are provided in Appendix J of 10CFR50.

Steel is a dimensionally and chemically stable material in a benign environment. However, the strength and stiffness properties of steel structures may degrade over time in hostile service environments from corrosion, metallic fatigue or crack propagation (especially in welds), or metallurgical changes in the steel. Such degradation mechanisms may arise from mechanical or thermal loads from service or extreme environmental loads, particularly those causing cyclic inelastic deformations, thermal gradients or cycling, aggressive chemical attack and irradiation. Operation at elevated temperatures tends to accelerate the 
degradation processes. Moreover, operation at prolonged elevated temperatures can lead to synergistic effects and accelerated damage (e.g. between creep and fatigue damage) that might not be apparent at lower temperatures (Jaske, 1987). Steel structures that function in an aggressive environment require occasional inspection and maintenance or repair to maintain their performance and reliability at an acceptable level (e.g., Banon, 1994b).

An evaluation of the reliability of a steel containment or liner for a period of continued service requires, first of all, a knowledge of its initial design and construction. Challenges to its strength from its service history also must be taken into account. The condition assessment and damage analysis methodologies must relate the significant material aging factors, environmental effects and structural loads to engineering properties that are needed for customary structural behavior evaluation and safety assessment. Finally, timedependent strength and stiffness degradation, load history and inspection/maintenance policies must be integrated into a decision tool to evaluate current and future safety or serviceability margins and to support rational policy development. This decision tool should take into account the stochastic nature of past and future loads due to operating conditions and the environment, randomness in strength, and uncertainty in nondestructive evaluation techniques. With these decision tools, the following issues could be addressed:

1. What aging factors are significant for steel containments and liners in terms of their future reliability?

2. Has the original strength of the structure degraded over time as a result of corrosion, fatigue/crack growth, elevated operating temperatures, thermal cycling, or irradiation?

3. What is the residual structural safety margin or residual life of the containment and how would it respond to a design-basis event?

4. Which NDE techniques (e.g., ultrasonic, acoustic emission, radiography) or in situ strength measurement methods are most useful for locating strength-degrading defects and for demonstrating reliability of an existing containment?

5. What inspection procedures should be required, how frequently should they be conducted, and what statistically-based sampling plans should be implemented to provide the needed evidence of reliability?

Structural reliability analysis methods provide the logical framework for decision analysis in the presence of uncertainty (Melchers, 1987; Yao, 1986). Probability-based methods and technical data to support condition assessment have been developed for concrete structures in NPPs (Naus, et al, 1993; Ellingwood, and Mori, 1992; Mori and Ellingwood, 1993, 1994a, 1994b). Similar methods are required for steel containments and liners. Some rudimentary methods for making a quantitative evaluation of the residual strength or remaining service life of a steel structure based on a knowledge of its service history, present condition, and projected use during a period of continued service already exist (Kameda and Koike, 1975; Ellingwood, 1976; Committee, 1982; DeKraker, et al, 1982; Siemes, et al, 1985; CIB, 1987; Ellingwood and Mori, 1992). Further development of such methods and their adaptation in decision-making for steel containments and liners are the subject of the proposed research.

Structural condition assessment may be required by the regulatory authority as a basis of criteria for facility risk management. As an additional benefit, it also provides a NPP operator with cost-effective risk management and decision-making tools. Such tools focus management attention on significant risk contributors and minimize expenditures on items that have a negligible contribution to risk, thus optimizing efforts to maintain safety at a minimum cost. 


\subsection{Project goals, objectives and scope}

The overall goal of the research is to develop a methodology to permit quantitative assessments of current and future structural reliability of steel containments and liners in NPPs, taking into account service conditions and environmental factors that might diminish their residual safety margins during future designbasis events. This goal is supported by the following project objectives:

1. Identify mathematical models from principles of structural mechanics to evaluate degradation in strength of steel structures over time in terms of initial construction conditions, service load history, and aggressive environmental factors.

2. Recommend statistically-based sampling plans for nondestructive evaluation (NDE) of steel structures to ensure that damage due to corrosion, fatigue cracking or other factors is detected with a specified level of confidence.

3. Develop methods to assess the probability that steel containment or liner capacity has degraded below a specified level or will do so during a future service period, taking into account initial conditions of the structure, service history, aging, nondestructive evaluation techniques, and in-service inspection/maintenance strategies.

The focus of the research is on steel containments, liners and other safety-related structural components and systems. Mechanical or electrical systems are not considered. It is assumed that the strength degradation and damage accumulation models and experimental data needed to support the structural reliability analysis either are available or will be developed in concurrent research activities conducted in other tasks of the program. The research does not involve experimental testing.

\subsection{Organization of report}

This report summarizes the first phase of the research. Predictive models are identified from principles of structural mechanics for assessing damage accumulation, residual strength and service life of steel containments and liners. Capabilities of current nondestructive evaluation methods are reviewed. Existing structural loading data are summarized. Time-dependent reliability analysis methods for in-service condition assessment are introduced.

Section 2 reviews degradation mechanisms that are potential contributors to deterioration of strength or stiffness of steel structures in general and containments and liners in particular. Mathematical models are presented for analyzing structural degradation over time.

Section 3 reviews common nondestructive evaluation techniques, with specific regard to characteristics that would be incorporated in a time-dependent reliability analysis or probability-based condition assessment.

Section 4 reviews fundamental probabilistic methods for analyzing time-dependent reliability of steel containments and liners in terms of component fragility, time-dependent limit state probability of failure, and cumulative probability of acceptable performance over a prescribed service interval.

Section 5 discusses the role of in-service inspection, nondestructive evaluation and maintenance in minimizing the impact of structural aging and in reliability assurance. Engineering decision analysis enables competing maintenance strategies to be evaluated in terms of risk and cost.

Section 6 presents a Markov model for tracking systematically the evolution of states of damage in 
a structure throughout its service life, including periodic in-service inspection and maintenance. Such a model would facilitate computerization of damage accumulation history and could provide an audit trail of facility risk management over a service life.

Section 7 presents recommendations for further work.

Section 8 lists references on condition assessment and reliability-based service life prediction of containments and liners. 


\section{STRUCTURAL DETERIORATION AND ITS EVALUATION}

A steel containment or liner of a concrete containment functions as a pressure-retaining boundary and as a barrier to the release of radionuclides to the environment. The containment is among the components most critical for public safety in a NPP. The general design criteria in Appendix A to 10CFR50 provide minimum requirements for design, fabrication, construction, testing and inspection of steel containments and liners. Periodic testing to ensure leaktightness of the containment, resilient seals and bellows is required. A revision to Appendix J, issued September 26, 1995, will allow qualified licensees to perform leakage rate tests less frequently than previously required. Rules for design and in-service inspection of containments and liners are also contained in the ASME Boiler and Pressure Vessel Code Sections III and XI, respectively.

The configuration of the containment or liner depends on the type of containment and pressure suppression system. For structural evaluation purposes, the containment can be considered to be a thin [less than 2 in $(51 \mathrm{~mm})$ ] cylindrical/spherical/ellipsoidal steel shell with numerous penetrations for piping and venting. The shell diameter can range anywhere from about $35 \mathrm{ft}(10.7 \mathrm{~m})$ to $140 \mathrm{ft}(42.7 \mathrm{~m})$, while the height varies from $115 \mathrm{ft}(35 \mathrm{~m})$ to $240 \mathrm{ft}(73.2 \mathrm{~m})$. A typical containment shell is low-carbon steel such as ASTM A-516 Grade 70, with a yield strength of $38 \mathrm{ksi}(262 \mathrm{MPa}$ ) and tensile strength of $70 \mathrm{ksi}(483 \mathrm{MPa})$. The penetrations for high-temperature piping are equipped with stainless steel bellows to permit thermal expansion without unduly stressing the shell. The bellows typically are two-ply Type 304 stainless steel, with each ply $0.6-0.9 \mathrm{~mm}$ in thickness, with minimum yield strength and tensile strength of $30 \mathrm{ksi}(207 \mathrm{MPa})$ and $75 \mathrm{ksi}$ (517 MPa), respectively. The cold-rolling process leaves high residual stresses. The metal shell and all penetration assemblies, piping, pumps and valves required to isolate the system and provide a pressure boundary constitute the primary containment system.

Degradation in engineering properties of steel containments and liners is caused by mechanical and thermal loads, which may occur in corrosive and embrittling environments. Reviews of operating power plants have revealed a number of mechanisms of deterioration that may lead to degradation of strength and stiffness of steel containments or liners (Shah and MacDonald, 1989; Shah, et al, 1994). The environment within containments generally is humid and hot $[40-60 \% \mathrm{RH}, 66 \mathrm{C}]$. Generally, steels in areas where water or condensation accumulates or that are exposed to aggressive chemicals such as borated water in PWRs, sodium pentaborate in BWRs, and decontamination fluids may be susceptible to corrosion. Embedded shell regions of drywells also are susceptible to corrosion. In PWR plants with steel containments or liners, corrosion has been observed on the outside of the steel shell in the annular region or where the shell is embedded in the concrete basemat. The exterior of the drywell in Mark I containments is susceptible to general, pitting and crevice corrosion when wet or degraded fill material is present in the gap between the shield and drywell. Pressure suppression chambers are susceptible to general and pitting corrosion in the vicinity of the waterline, especially when the coatings deteriorate. Corrosion damage has been found in the containments at the McGuire, Oyster Creek, Catawba and Nine Mile Point plants. A recently published review (Oland and Naus, 1996) indicates that out of 37 instances of degradation reported, 18 involved corrosion of the shell or liner. Low-cycle fatigue may occur at geometric discontinuities and penetrations from cyclic thermal or mechanical loads from operating transients, pressure tests, and safety relief valve (SRV) discharge tests. Transgranular stress-corrosion cracking or corrosion-fatigue due to high residual stresses, stress concentrations, and misalignment may be a problem in stainless steel bellows, where some instances of leakage have been reported. Bolting fatigue, wear and corrosion and deterioration at flashed, caulked or sealed joints all have been noted.

Operation at elevated temperatures often accelerates the damage accumulation process. Moreover, high temperatures cause other effects to be synergistic: interaction of creep and fatigue at high temperature is greatly accelerated. Accelerated testing may lead to a pessimistic appraisal of service life; e.g., overstressing accelerates degradation due to creep. 
The major mechanism of deterioration affecting steel structures in NPPs thus appears to be corrosion, with fatigue or corrosion/fatigue possible but less likely. Accordingly, these mechanisms are described in detail in the following sections. Since the probability distributions of damage or residual strength are required for time-dependent reliability analysis, statistical descriptions of damage parameters also are provided, where available.

\subsection{Corrosion}

Corrosion is an electrochemical reaction between a metal and its environment. Corrosion is the most damaging mechanism affecting metal containments and liners. Mechanisms that are of particular significance in carbon steels used for NPP containments, liners and other Category I steel structures are uniform corrosion, localized pitting and crevice corrosion. Intergranular or transgranular stress-corrosion may also occur, and may be important in stainless steels. Corrosion impacts one structural limit state and one performance-related limit state. At design load conditions, shell thinning from general corrosion may lead to gross inelastic deformations in regions of tensile stress or instability in regions of compressive stress. Penetration of the shell by localized corrosion may lead to the development of a leak path and diminished pressure retention.

The electrochemistry of the corrosion process is reasonably well understood, and mathematical models of the electrochemical processes underlying corrosion are available (Berger, 1983). Here, we emphasize those aspects of the corrosion process that impact structural performance.

\subsubsection{General or uniform corrosion}

Uniform corrosion occurs over a large area of the surface of the component and is characterized by an essentially uniform thinning of the section. Excessive thinning due to uniform corrosion may lead to gross inelastic deformations or instability of the shell. The depth of corrosion in steel is modeled by,

$$
x(t)=C\left(t-t_{i}\right)^{a}
$$

in which $t=$ time, $t_{i}=$ induction or initiation time required to activate the process, $C=$ rate parameter, and $\alpha$ $=$ time-order parameter. It should be noted that Eqn 2.1 is empirical in nature. The associated corrosion rate (for purposes of comparison with experimental data) is,

$$
\mathrm{dx} / \mathrm{dt}=\alpha \mathrm{C}\left(\mathrm{t}-\mathrm{t}_{\mathrm{i}}\right)^{(\alpha-1)}
$$

The parameters $\mathrm{C}$ and $\alpha$ must be determined from experimental data, supplemented by knowledge of the physics of the underlying mechanism of attack. For example, if the mechanism is diffusion-controlled, then $\alpha=0.5$. (In time-dependent reliability analysis, $\mathrm{C}, \alpha$ and $\mathrm{t}_{\mathbf{i}}$ are modeled as random variables, as described subsequently).

An alternate expression for corrosion is (Porter, et al, 1994),

$$
x(t)=a \ln (t)
$$

in which a is an experimental constant and the induction time has been ignored. The implied corrosion rate is proportional to $1 / t$.

Two general methods are recognized for estimating atmospheric corrosion-resistance of low-alloy steels (ASTM G101, 1989) from test data. The first utilizes linear regression analysis of short-term data to predict long-term performance by extrapolation. The second determines a corrosion resistance index based on chemical composition of the steel. The regression analysis presumes a log-linear relation between loss and 
time, leading to Eqn 2.1. The time-order parameter $\alpha$ is invariably less than unity, indicating a decrease in corrosion rate with time. The idea of extrapolating beyond the realm of observed data violates one of the basic tenets of regression analysis as a predictor. No convincing answer is provided to the question of long-term extrapolation or similitude of accelerated aging testing.

Corrosion testing is conducted mainly in the laboratory under carefully controlled conditions with small specimens. Corrosion progression normally is measured by weighing and measuring material loss. Laboratory experiments often involve accelerated testing, or attempts to simulate a multi-year service life by a test of a few weeks or months. One must be cautious in using the results of such accelerated aging tests to determine $C$ and $\alpha$, as the aging mechanisms may not scale properly from the laboratory to the service environment (Jaske, 1987; Natalie, 1987; Clifton, 1993). Physical factors that govern the corrosion process include temperature, residual stress, and cyclic loading rate, if cyclic loads are present. Temperature affects oxygen solubility, $\mathrm{pH}$, and corrosion product formation. In the presence of a moving fluid, the corrosion rate may increase as fluid velocity increases. Degree of exposure - total, partial, or intermittent - also may change rate and mode of corrosion. On the other hand, the induction period normally is ignored in an accelerated laboratory test. Failure to include this (random) induction time has been shown to lead to a conservative estimate of remaining service life or residual strength (Ellingwood and Mori, 1992).

Corrosion testing occasionally may be conducted in the field. Field tests may involve either small specimens or structural components. While environmental similitude is easier to maintain, accurate measurements of the corrosion process may be difficult to obtain under field conditions.

Table $2.1^{*}$ summarizes average uniform corrosion parameters for carbon and weathering steels, some of which are similar to the low-carbon ferritic steels used for containments and liners, in several environments (Komp, 1987; Structural, 1989). These values were determined from tests of small specimens, and some error may result from extrapolating these data to structural members. The constants $C$ and $\alpha$ are such that $x(t)$ is measured in $\mu-\mathrm{m}$ when $\mathrm{t}$ is measured in years. Since no information or data were provided on the corrosion induction period, it was assumed that corrosion initiated immediately and that $t_{i}=0$; this is a conservative assumption. Some of the parameters provided by Komp (1987) have been used in reliability-based evaluation of bridge deterioration (Kayser and Nowak, 1989) and to devise bridge inspection strategies (Sommer, et al, 1993). Uniform corrosion rates are dependent on the environment and ambient temperature. The uncertainty in the corrosion rate is quite large; one reference reported a coefficient of variation of 0.7 for uniform corrosion in stainless steel containers (Porter, et al, 1994). A more typical coefficient of variation in $\mathrm{C}$ would be 0.3 .

The time order parameter for uniform corrosion, $\alpha$, is less than unity, indicating a decrease in corrosion rate with time. The initial corrosion rate in mild steels exposed to fresh or seawater is of the order $200 \mu-\mathrm{m} / \mathrm{yr}(0.2 \mathrm{~mm} / \mathrm{yr})$, decreasing parabolically to $100 \mu-\mathrm{m} / \mathrm{yr}$ after one year (Akashi, et al, 1990) as corrosion product film provides a protective barrier against further oxidation. The time-order parameter can be treated as deterministic; its proximity to 0.5 suggests that corrosion might be modeled as a diffusion-type process.

In NPPs, estimated general corrosion rates from field surveys are (Shah, et al, 1994): 0.52 - $1.4 \mathrm{~mm} / \mathrm{yr}$ (Oyster Creek exterior drywell shell); $0.08 \mathrm{~mm} / \mathrm{yr}$ (Nine Mile Point torus interior above waterline); $1.15 \mathrm{~mm} / \mathrm{yr}$ (McGuire 2, exterior of the containment); $0.33 \mathrm{~mm} / \mathrm{yr}$ (McGuire 1 interior of containment). One must be cautious about extrapolating such measurements to service life prediction since the corrosion rate decreases with time (cf Eqns 2.2 and 2.3), and corrosion measured early in a service period may not be indicative of subsequent performance. Coating degradation from temperature, condensation and immersion, radiation and impact allows corrosion to initiate and spread, lifting the coating and accelerating deterioration.

- Tables and figures are placed at the end of each section. 
Possible structural degradation from uniform corrosion often is addressed in ordinary structural design by providing an extra thickness or "corrosion allowance" to the material. This allowance typically is on the order of $1-3 \mathrm{~mm}(1 / 16-1 / 8 \mathrm{in})$ when no protective coating is provided. The maximum penetration of corrosion is a random variable and can be modeled by a Type I distribution of largest values or Gumbel distribution (Akashi, et al, 1990). Parameters of the extreme value distribution can be related empirically to the mean corrosion penetration; this then can be used to determine the 99-percentile value of loss of section due to corrosion and thus a corrosion allowance (in $\mathrm{mm}$ ) to ensure satisfactory performance during a service period.

The corrosion allowance approach has also been suggested for designing containers for long-term waste storage (Marsh, 1985). Problems with long term extrapolation of data (out to 1000 years or more) necessitates very conservative assumptions regarding corrosion mechanisms. Making such assumptions, steady-state corrosion rate in low-carbon steel at $90 \mathrm{C}$ is predicted to be $209 \mu-\mathrm{m} / \mathrm{yr}$. Experimental data (shortterm) invariably fall below this level.

The presence of aggressive chemicals (e.g., boric acid, sodium pentaborate) can accelerate the rate of metallic corrosion (Czajkowski, 1990). Components known to have been affected by corrosive attack by borated water leaking through seals and valves include threaded fasteners, reactor coolant piping, pumps and valves. Corrosion reported at the Catawba and McGuire plants was due to borated water leaking from an instrumentation line which pooled against the metal shell. Corrosion rates up to 1.7 inches/yr ( $43 \mathrm{~mm} / \mathrm{yr})$ may occur in carbon or low-alloy steels exposed to borated water at $200 \mathrm{~F}(92 \mathrm{C})$; because of the high rate, such components cannot be designed using the corrosion allowance approach, and instead must be protected from such aggressive attack.

\subsubsection{Localized corrosion - pitting and crevice}

Pitting and crevice corrosion are highly localized. Pits can be hidden under a surface of corrosion products, making detection difficult. Many nondestructive methods can locate relatively large pits but cannot distinguish between pits and other surficial defects (Sprowls, 1987). Pitting corrosion is often identified by the presence of surface nodulation. Problem areas usually represent only a small percentage of total surface area, and the local pitting usually is not accompanied by significant loss of material. However, evaluation of the depths of pitting corrosion is necessary to ensure the integrity of the pressure boundary. A single throughthe-thickness crack is sufficient to cause leakage.

Pitting and crevice corrosion are similar in their mechanisms and descriptive mathematical models (Sprowls, 1987; Sharland, et al, 1989). The pitting process appears to be initiated by an electrochemical breakdown of the passive film from local acidity, inhomogeneities in the material, or other phenomena causing local disruption of the passive layer. Cyclic loading also can disrupt the passive layer, forming anodic areas at points of rupture and giving rise to corrosion-fatigue.

The initiation and growth of pits are not readily measured by methods that are used to evaluate uniform corrosion. In fact, pits frequently become dormant following an initial period of growth and subsequently reinitiate (so-called pit birth and death - Williams, et al, 1985). However, mathematical modeling of growth of individual pits follows the same semi-empirical formulas as used for uniform corrosion (Eqns 2.1 - 2.3). In aluminum, it has been observed that pit depth is proportional to $t^{1 / 3}$ (Sprowls, 1987). In steels, it has been observed (Kondo, 1989) that pit volume increases linearly with time. Assuming a hemispherical pit of radius $r$ and constant bulk dissolution rate $B$, pit volume $(2 / 3) \pi r^{3}=B t$, again implying that $r$ is proportional to $t^{1 / 3}$. This seems to agree well with experimental results. On the other hand, at least one study (Porter, et al, 1994) suggests that in stainless steels, pit growth is a linear function of time. Ahammed and Melchers (1995) proposed a pitting rate proportional to $t^{-0.6}$. The pitting corrosion rate can be $3 \times 10^{5}$ to $10^{6}$ times higher than general corrosion (Joshi, 1994). Shibata (1994) reports an exponent of 3.42 in Eqn 2.1. 
Local pitting corrosion penetration is related to anodic current density (Marsh, et al, 1985). Maximum pit depths were measured over an area of approx $3 \mathrm{~m}^{2}$ in carbon steel specimens tested in $\mathrm{NaHCO}_{3}+\mathrm{Cl}^{-}$for periods of up to 1.1 years. These data were analyzed at different exposure times using statistics of extremes. Results indicated that maximum pitting depth was related to time by $x_{\max }=8.35 \mathrm{t}^{0.46}$, where $t$ is in $y r$ and $x_{\max }$ in mm. Extrapolation of these data to a 1000-yr life yielded a pit depth of $200 \mathrm{~mm}$; however, the validity of this extrapolation clearly is questionable and without doubt conservative.

Table 2.2 summarizes data on pitting corrosion identified by a literature search. Although only limited data were identified, it is clear that the rate of pit growth is potentially much higher than that of uniform corrosion.

Limited statistical studies have been performed for pitting corrosion depth. When several pits are present, the maximum pit depth $\mathrm{x}_{\max }$ within an area is of more interest than the distribution of individual pit depths. $x_{\max }$ has been reported to be a linear function of the log of exposed area (Aziz, 1956; Joshi, 1994). The distribution of maximum pit size can be determined from the individual pit depths using extreme value statistics (Sprowls, 1987; Kondo, 1989), assuming that the pit depths are statistically independent (Joshi, 1994; Scarf and Laycock, 1994; Shibata, 1994).

Mola, et al (1990) developed a stochastic model for pitting corrosion. They assumed that the number, $N(t)$, of pits at time, $t$, is a Poisson process, dependent on the mean surface area and random initiation time. The growth in pit surface area is described by a stochastic finite difference equation. Provan and Rodriguez (1989) modeled the growth in maximum pit depth as a discrete-space, continuous-time Markov process. The evolution of the probability density of pit depth in time was described by a Kolmogorov forward differential equation. A laboratory program conducted as part of this study found that the mean and variance of maximum pit depth were proportional to $t^{\mathrm{a}}$ and $\mathrm{t}^{\mathrm{b}}$, respectively, where $0<\mathrm{a}, \mathrm{b}<1$. The probability distribution of maximum pit depth was found to be Type I extreme value at different exposure periods, with mode and scale parameters that increase linearly with time (Strutt, et al, 1985).

In a electrochemical rather than structural engineering approach (Gabrielli, et al, 1990), changes in current during corrosion were measured and analyzed statistically. The "survival probability" was the probability that the electrode remained unpitted. The probability of survival was found to be,

$$
L(t)=\exp (-A(t) t)
$$

in which $A(t)=$ time-dependent pit generation rate. If the surface area is divided into small elementary areas, pitting in each area can be treated as statistically independent events. The maximum pit depth was described by a Type I distribution of largest values. If depth increases by $x=b \log t+c$, then $d x / d t=b / t$ and time at which the maxmum pit penetrates the thickness of the component is described by a Weibull probability distribution. This time-dependent model does not take into account birth and death processes of pits. Moreover, pit initiation cannot be modeled as a Poisson process with stationary increments, since the intervals are not independent and occurrences have a tendency to cluster.

\subsubsection{Deterioration of coatings}

Coatings protect the structure from corrosion and facilitate decontamination. Coating degradation can occur due to elevated temperatures, excessive moisture, radiation, and mechanical abrasion and chipping. Localized problems occur before general failure of the coating system. Once corrosion initiates, however, failure of the coating system accelerates.

Many plant owners already have found it necessary to perform local repairs of coating systems, and it seems likely that such repairs will continue to be needed at regular intervals during an extended service life. 
Fortunately, coating maintenance usually can be performed along with other required maintanance activities. Underwater-cured epoxy is a common solution for spot repairs of coatings in tanks and supression chambers. Coating performance thus does not appear to be a significant consideration in developing reliability-based condition assessment methodologies.

\subsection{Fatigue and fracture}

Metals contain voids and inclusions at the microscopic level. In addition, a structural component may contain surficial geometric discontinuities (weld undercuts, reentrant corners, holes) or local damage (cracks, corrosion loss) that cause stress concentrations. In the presence of cyclic loads, these subcritical defects may grow and cause fatigue in the metal, eventually leading to failure at loads much smaller than the statically applied monotonic load causing failure.

The loads applied to a NPP structure may be cyclic in nature. Sources of operational cyclic thermal and mechanical loads in a containment include startup/shutdown thermal transients, pipe reactions, SRV discharge test loads, crane and refueling loads. Although extreme environmental events such as earthquakes may also induce cycling, the rate of occurrence and duration of such events is sufficiently small that they would not cause fatigue damage to accumulate.

Early NPP steel structures and components were designed with little consideration for fatigue. Since the late 1960's, however, design requirements for RPVs and Class 1 piping have included fatigue considerations (Ware, et al, 1995). Current fatigue design analyses are aimed at demonstrating that a component has a cumulative use factor (computed from a Palmgren-Miner analysis, to be described subsequently) of less than 1.0 at the end of a 40-year design life. The analysis is made with conservative assumptions regarding the number and magnitude of operating transients. Several fatigue monitoring programs are under development in the U. S., aimed at determining increases in the cumulative usage factor on-line as a function of operating transients.

Fatigue is not believed to be a significant problem in steel containments and liners except at points of structural discontinuities (weld undercuts, etc.), or heavy weldments where residual stresses may approach yield. Most full-penetration thick welds in NPP containments are stress-relieved, so residual stresses are not a problem at such sites. Ductile carbon steels of the type used in containments and liners are not susceptible to low-cycle fatigue, and can withstand numerous reversals of moderate inelastic strain without failure. However, general corrosion may cause the surface of the shell to become rough, causing local stress concentrations, and corrosion pits also may serve as sites for fatigue crack initiation and growth. Crack initiation time can be reduced by a factor of as much as three when pitting is present. An exception to the general fatigue insensitivity of the containment is the stainless steel bellows at Mark I containment penetrations, which have high residual stresses from cold-rolling and are susceptible to low-cycle fatigue and stress-corrosion cracking.

It is convenient to envision three stages in the fatigue process: (1) crack initiation; (2) stable (subcritical) crack growth; and (3) unstable crack growth or fast fracture. The third stage occurs so rapidly in comparison with the first two that it can be ignored in service life predictions. Crack initiation and growth processes are driven by different factors. The initiation phase reflects interactions of the metal with the bulk environment. Dislocations due to slip lead to highly localized stresses that nucleate a macroscopic crack that then propagates. In the crack growth phase, the local crack tip environment, which may be different from bulk environment, determines the process. The relative contributions of these phases depend on the load spectrum, material characteristics, and initial condition of the component. If the structure is essentially defect-free and the stress range is low, most of the life of the structure is consumed in initiating a detectable flaw. On the other hand, many welded components contain initial flaws (lack of fusion, penetration), and in such components, there is essentially no initiation phase. A facility for analyzing both phases of fatigue is required in condition 
assessment. Fatigue often is thought of as having two domains: (1) low-cycle fatigue, with service life of 100,000 cycles or less, and (2) high-cycle fatigue, with service life of more than 100,000 cycles. In the latter, the metal initially is essentially defect-free and cyclic stresses remain in the elastic range.

During a service load history involving time-varying or cyclic loads, failure can occur by single overload or by accumulation of damage (Madsen, 1982). In failure analysis, "damage" is an aggregated parameter describing the macroscopic appearance or manifestation of functional impairment. There may be no immediate relation between damage and measurable physical quantities (this is the case prior to visible crack initiation, where microstructural changes cannot be detected by the usual field inspection methods), or the relation may be quite obvious (crack propagation). Time-dependent reliability methods and condition assessment procedures must be tailored to these realities. Sources of uncertainty in fatigue modeling include random load and material properties, system modeling, damage accumulation law, and defect size. The state of the art of probabilistic fatigue analysis was reviewed in a series of four papers by the Committee on Fatigue and Fracture Reliability (Committee, 1982).

\subsubsection{Low-cycle fatigue}

All models used to analyze fatigue are empirical to a degree, with parameters that are dependent on the metal and its service environment and are determined by testing small specimens under cyclic load. The primary load parameter affecting fatigue is the stress (or strain) range, $\Delta S=S_{\max }-S_{\min }$. Other factors that may be important in varying degrees include mean stress (or stress ratio, $R=S_{\min } / S_{\max }$ ), load sequence, and cyclic frequency. Fatigue life to "failure" is defined in a number of ways: as time (or number of cycles) to complete fracture of the specimen; as time required for a specified increase in specimen compliance; or as time to initiation of detectable (and presumably repairable) cracking. When utilizing experimental data to develop fatigue assessment procedures, it is essential to understand the relation between "failure" as it relates to the peformance of the structure in service and "failure" as it is defined in the experimental fatigue database. It is surprising how often analysts ignore these differences.

The most common way of expressing the fatigue life of a component in terms of the number of cycles to "failure," $\mathrm{N}$, is through the well-known $\mathrm{S}-\mathrm{N}$ relation between stress and cycles,

$$
N(\Delta S)^{m}=C
$$

in which $\Delta \mathrm{S}=$ applied stress range and $\mathrm{m}$ and $\mathrm{C}$ are experimental constants. When the fatigue testing is deformation-controlled rather than load-controlled, stress range is often replaced with total strain range or plastic strain range. Eqn 2.5 is sometimes referred to as the Basquin equation. A more general model is the Coffin-Manson equation (discussed in Committee, 1982)

$$
\Delta \epsilon / 2=\left(\sigma_{f} / E\right)(2 N)^{b}+\epsilon_{f}(2 N)^{c}
$$

in which $\Delta \epsilon=$ strain range, $\mathrm{E}=$ modulus of elasticity, and $\sigma_{\mathrm{f}}, \epsilon_{\mathrm{f}}, \mathrm{b}$ and $\mathrm{c}=$ experimental constants. The first term is equivalent to Eqn 2.5, expressed in terms of elastic strain; the second term dominates in the low-cycle regime, where the cycling is inelastic. Several typical S-N curves used in design are illustrated in Figure 2.1. They are based on different testing conditions and load cycling. However, the tests were conducted in air. The curves labeled AISC/AASHTO B and D are based on fatigue tests of welded details found in buildings and bridges, and cycling was load-controlled and mainly from zero to maximum tension $(R=0)$. Curves AWS-X, API-X and DEn are found in design guides developed by the American Welding Society, America Petroleum Institute, and the United Kingdom Department of Energy, respectively. In contrast, the curves labeled "ASME mean" and "ASME design" are based on tests of small smooth polished specimens tested with fully reversed strain-controlled cycling $(R=-1)$. The ASME curves plot stress amplitude, computed as $S_{a}=E \Delta \in / 2$ in which $\Delta \epsilon$ is strain ranged defined in Eqn 2.6. Note that the exponent $m$ is approximately 3 in all cases; in a corrosive 
environment, $\mathrm{m}$ increases to the range $3.5-4.0$. The rate parameter, $\mathrm{C}$, is dependent on the specimen geometry, yield strength of the material, and frequency at which the cyclic load is applied. Interestingly, the dependency of $\mathrm{C}$ on yield strength is not especially strong, implying that increases in yield strength and fatigue strength are not commensurate with each other.

Fatigue test data presented in the form of S-N curves indicate a substantial inherent variability about the median curve; coefficients of variation in fatigue life at a given stress commonly are on the order of 0.30 0.60 (Committee, 1982). This inherent variability can be displayed by presenting the S-N curves as a family of curves at different cumulative probabilities, or P-S-N curves (Provan, 1987). In developing fatigue curves for design purposes, one might select a 5 percentile or 10 percentile curve. However, in developing fatigue curves for design purposes from experimental data, it has been customary to divide the stress and/or corresponding median life by deterministic factors of safety. For example, in ASME Code Section III, median low-cycle (controlled strain) fatigue curves were lowered by factors of 2 on stress and 20 on cycles to obtain design fatigue curves. These adjustments are intended to account for scatter in data, size effects, surface finish, and environmental effects. More recent studies indicate that these ASME curves may not address environmental effects in the low-cycle (strain greater than 0.1\%) range (Keisler, et al, 1994). Data from several samples of smooth specimens tested under fully reversed strain cycling $(R=-1)$ were analyzed. Temperature (in air), percentage dissolved oxygen and strain rate (in aqueous solution) had the most significant impact. Strain rate in air or characteristics of load vs time had little effect on fatigue life.

Because of economic limitations, most fatigue data are determined by testing small, smooth specimens under carefully controlled conditions. Most structural components that are susceptible to fatigue damage are neither small and smooth nor subject to constant amplitude cycling. Fatigue damage is most likely to initiate and develop at weld undercuts and other stress raisers (notches) where the local stress (or strain) is amplified by a significant factor above the "far-field stress" (or strain) computed from a finite element analysis. Such local effects are not included within the normal factors of safety on smooth-specimen fatigue curves alluded to above. The question arises as to how to deal with such local effects.

One approach is to conduct fatigue tests of larger specimens containing representations of the fatiguecritical structural detail. This has, in fact, been done for civil structures; over a period of three decades from the 1950 's to the 1980 's, numerous fatigue tests of representative bolted and welded details mainly representative for bridge structures were conducted at Lehigh University, the University of Illinois, and other institutions (reviewed by Keating and Fisher, 1986). The test results were collected in six main categories of details (curves for two categories - B and D - are illustrated in Figure 2.1), and allowable cyclic stresses for four main load conditions were determined with an appropriate factor of safety. The results can be seen in Appendix K of the new LRFD Specification (LRFD, 1993). Such an approach, while acceptable for routine design of civil structures where details are repetitive, may be unduly conservative when applied to a specific fatigue-critical detail. Thus, its use in condition assessment of a set of specific details in a structural system may not provide uniform or consistent reliability among these details. Moreover, in light of recent advances in computational ability to analyze complex nonlinear stress-strain histories accurately, it is a highly inefficient method for condition assessment.

Smooth-specimen fatigue curves can be used to determine fatigue behavior of structural components with stress raisers, provided that the local stress-strain history at the notch can be analyzed. If the material remains entirely elastic, the local maximum stress (or strain) at a notch is the product of the far-field stress and a stress concentration factor, $K_{t}$ However, when the material local to the notch is stressed beyond the elastic range, the local stresses and strains can no longer be determined from $\mathrm{K}_{t}$. Studies (Ellingwood, 1976) have shown that Neuber's rule (Topper, et al,1969) can be used in this case to determine the local stress-strain history at the notch needed to utilize smooth-specimen fatigue data. Neuber's rule postulates that the product of local stress and strain is proportional to the product of far-field elastic stress and strain, or, 


$$
\Delta \sigma \Delta \varepsilon=k_{f}^{2} \Delta S \Delta e
$$

in which $k_{f}$ is an effective stress concentration factor and the elastic stress, $\Delta S$, can be determined by finite element analysis or other conventional structural analysis procedure. The second condition needed for determining $\Delta \sigma$ and $\Delta \epsilon$ is given by the constitutive model for the material. Assuming, for example, a Ramberg-Osgood law,

$$
\epsilon=\mathrm{K}(\boldsymbol{\sigma})^{\mathrm{n}}
$$

in which $\mathrm{K}=$ compliance and $\mathrm{n}=$ strain-hardening exponent, one would obtain,

$$
\Delta \sigma=\left[\left(k_{f} \Delta S\right)^{2} / K E\right]^{\frac{1}{n \cdot 1}}
$$

Entering the S-N curve at this value of $\Delta \sigma$ would give the fatigue life of the detail.

When the load amplitudes vary in time, the number of cycles to failure (or time to failure) must be determined from a cumulative damage law. Such laws relate fatigue behavior under variable amplitude loading to the known behavior under constant amplitude cycling which can be determined from experiments. The most commonly used of these laws is the Palmgren-Miner hypothesis, which postulates that damage accumulates linearly simply as a function of the number of cycles at a particular stress (or strain) level. Under variable amplitude loading, then, damage accumulation, $\mathrm{D}$, is described by,

$$
D=\sum_{i} \Delta D_{i}=\sum_{i=1}^{N} C^{-1}\left(\Delta S_{i}\right)^{m}
$$

in which $N=$ number of load cycles and, $\Delta D_{i}=$ increment of damage in cycle $i$. If the load history consists of $\mathrm{k}$ discrete load amplitudes, Eqn 2.10 takes the more familiar form,

$$
D=\sum_{i=1}^{k} n_{i} / N\left(\Delta S_{i}\right)
$$

in which $n_{i}=$ number of cycles in the load history at stress level $\Delta S_{i}, N\left(\Delta S_{i}\right)=$ number of cycles to failure under constant amplitude loading $\Delta S_{i}$, determined from Eqns 2.5 or 2.6, and $\Sigma n_{i}=N$. When the cycles are not clearly defined, as is sometimes the case in broad-band excitation, rainflow cycle counting can be used to determine $n_{i}$ (Barson and Rolfe, 1987). (This necessitates a time-domain rather than frequency-domain analysis.) The Palmgren-Miner hypothesis asserts that failure occurs when $\mathrm{D}>1.0$.

The Palmgren-Miner damage hypothesis does not account for stress sequence effects on fatigue life. However, reviews of other damage accumulation theories indicate that other, more complex, rules do not provide consistently better results (Committee, 1982).

\subsubsection{Crack propagation and fracture}

Growth of an existing crack, once initiated, can be predicted by fracture mechanics analysis (Broek, 1988). Under the domain of applicability of linear elastic fracture mechanics (LEFM), unstable crack growth leading to fracture initiates when,

$$
\mathrm{K}>\mathrm{K}_{\mathrm{Ic}}
$$

in which $\mathrm{K}_{\mathrm{Ic}}=$ (plane strain) fracture toughness and $\mathrm{K}=$ stress intensity. The plane strain fracture toughness is a material-dependent parameter dependent on temperature, rate of loading, and the environment. For mild 
steel at ambient conditions, $\mathrm{K}_{\mathrm{rc}}$ typically is about $140 \mathrm{MNm}^{-3 / 2}$, and its coefficient of variation typically is about 0.15 . The stress intensity is,

$$
K=Y(a) \sigma \sqrt{\pi a}
$$

in which $\sigma=$ far-field stress, $a=$ characteristic crack size, and $Y(a)=$ correction factor based on relative crack size and shape and far-field loading. For a large plate in tension with a center crack (through the plate thickness) of length $2 a, Y(a)=1.0$; for a penny-shaped crack within the plate with radius $a, Y(a)=2 / \pi$; and for an edge crack loaded in tension or flexural tension with depth a less than half the thickness, $Y(a)=1.12$. Procedures are also available for modeling nonlinear behavior; the J-integral and crack-opening displacement approaches are two such procedures.

Fatigue crack growth (leading to increases in $\mathrm{K}_{\mathrm{I}}$ up to $\mathrm{K}_{\mathrm{Ic}}$ ) is most commonly modeled using the Paris equation (Barsom and Rolfe, 1987),

$$
\mathrm{da} / \mathrm{dN}=\mathrm{C}(\Delta \mathrm{K})^{\mathrm{m}}
$$

in which $\Delta \mathrm{K}=$ range of fluctuating stress intensity factor, obtained by replacing $\sigma$ by $\Delta \sigma$ in Eqn 2.13 , and $\mathrm{C}$ and $\mathrm{m}$ are experimental constants (unrelated to the $\mathrm{C}, \mathrm{m}$ in Eqn 2.5). With the stress history known, Eqn 2.14 can be integrated to determine the crack size as a function of elapsed cycles, $N$. Refinements to Eqn 2.14 for incorporating the mean stress or stress ratio, $S_{\min } / S_{\max }$, the threshold for crack growth, $K_{t h}$, and other factors known to affect crack growth rate, are available (Dowling, 1993). These effects generally have a second-order effect on predicted defect growth.

The crack growth analysis becomes difficult when several cracks grow simultaneously and interaction for cracks in close proximity may occur. In this case, the rate at which fractured area is produced may be more meaningful than crack growth rate. This suggests a "damage mechanics" approach, where multiple flaws are smeared (Kachanov, 1986).

Current analysis and design procedures do not consider possible synergistic effects of corrosion from an aggressive environment and fatigue from mechanical or thermal loads. The corrosion-fatigue process consists of several stages: pit formation and growth, crack formation from the pit (assuming corrosion pit can be modeled as a sharp crack), coalescence and corrosion-fatigue crack propagation (Kondo, 1989). Post-failure fractographic investigations have revealed that a pit (or pits) is often the origin of the fracture surface, meaning that pit initiation and coalescence is the trigger for fatigue crack initiation. The transition of a pit into a crack occurs at a stress intensity of about $\mathrm{K}=1.2 \mathrm{MPa}(\mathrm{m})^{1 / 2}$ for low-alloy steel; this is below the threshold for a long planar surface flaw (Kondo, 1989).

\subsubsection{Stress corrosion cracking}

Stress-corrosion cracking (SCC) and fatigue damage may occur in the bellows, which are subjected to reversals in deformations due to heating and cooling during normal operation, pressure loads during leak rate tests, and high residual stresses. Maximum bellows deformations are $13-50 \mathrm{~mm}$ due to thermal expansion; such cycles may occur on the order of 1000 - 5000 times during a 40-year service life.

The initiation of SCC on a surface appears to be primarily dependent on mechanically or chemicallyinduced rupture of the protective film (depassivation). This gives rise to acidity in occluded areas and development of local pitting or crevice corrosion at sites where cracking subsequently may initiate (Marsh, 1985; Kobayashi, et al, 1991). Stress-corrosion cracking can also initiate at sites within the interior of a component, even in the presence of an aggressive external environment. Interior microcrack formation appears to occur first where intergranular features are smooth rather than coarse, regardless of where such sites occur. 
The metal must be stressed in tension above a threshold stress or stress intensity level for SCC to initiate and progress. Once initiated, stress-corrosion crack growth occurs at a much higher rate than either general or pitting corrosion, and even the minimum rate is too great for a corrosion allowance approach to design.

SCC is troublesome for time-dependent reliability analysis. It is difficult to analyze mathematically and to detect or repair prior to component failure. There is no unique definition of when localized corrosion

changes into a stress-corrosion crack. Several cracks may initiate and repassivate prior to the time at which a dominant crack forms and propagates. Unexpected SCC problems in NPPs are costly to repair and raise additional safety concerns that are difficult to resolve. There are no satisfactory accelerated test methods (Kobayashi, et al, 1991) for SCC.

\subsection{Elevated temperature and irradiation effects}

Temperatures required for permanent degradation in strength or stiffness of carbon steel must be on the order of $500 \mathrm{C}$. Similarly, embrittlement of reactor pressure vessel steels has been noted for neutron radiation with fluence greater than $10^{19} \mathrm{n} / \mathrm{cm}^{2}$ (Chopra, et al, 1991). Since the containment is unlikely to see such levels of exposure, strength reduction due to prolonged elevated temperatures or radiation embrittlement of the metal containment shell is not generally a concern. Such effects may be of more, concern for the RPV and certain piping within the NPP. Neutron fluence of $10^{19} \mathrm{n} / \mathrm{cm}^{2}$ causes plane strain fracture toughness $K_{\mathrm{Ic}}$ in the reactor pressure vessel material to reduce by about 20\% during 40 years (Yoshimura, et al, 1993). Such fluences do not occur in the containment. On the other hand, creep can occur at elevated temperatures, introducing residual stresses or deformations (Murakami and Mizuno, 1991).

\subsection{Summary}

Rogers (1990) laments that "it is surprising that in the literature of corrosion failure prediction there are very few instances where statistical methods are applied." Commenting on fatigue crack growth two years earlier, Broek (1988) noted that "there are probably as many equations as there are researchers in the field," and "no equation can fit all data." There is substantial uncertainty, both inherent variability and modeling error, in modeling fatigue, corrosion, and their combinations, and in drawing inferences regarding their current and future impact on structural behavior.

Fatigue analysis methodology for predicting service life and margins of safety must rely heavily on small specimen testing coupled with advanced (nonlinear) computational procedures. There seems to be little prospect of using in-service data to develop models for predicting damage accumulation, other than in a qualitative sense. Failure rates in properly designed and fabricated structures are very small. Observations of in-service data to infer actual failure rates either involve extreme censoring of data or accelerated life testing. In an accelerated test, failure mechanisms may not scale as in the prototype. Extrapolation of such data is highly questionable. Moreover, early failures in service may derive from defects at assembly, error in fabrication, etc. It has been suggested (Strelec, 1993) that an "acceleration function" could be developed to make the time-dependent reliabilities the same under service and accelerated conditions. The acceleration function must be assumed; several have been proposed in which limited data have been used to estimate the empirical constants in the model statistically. However, this approach is empirical rather than mechanistic, as the scaling is done to preserve equal probabilities.

In modeling damage accumulation due to structural aging, it is important to measure microstructural parameters that correlate with an engineering property useful for structural evaluation. A review of the literature reveals that this often is not done, making much of the literature on material aging of limited use for structural evaluation purposes. Damage parameters in structural condition assessment must be defined to be consistent with detection parameters in common NDE methods. This is one difficulty in using the traditional S-N/Palmgren-Miner approach to analyzing fatigue. "Damage" in this approach evolves with cycles (or with 
time) in a way that cannot be measured by conventional NDE methods. As a result, the residual strength of a structure or component at some intermediate stage of damage evolution cannot be evaluated. Another example of this is the use of half-cell potential measurements to evaluate corrosion; this method can detect likely corrosion zones but cannot determine loss of section needed for structural calculations. In contrast, the detectability of a particular crack size can be related to the NDE method selected, as will be shown in Section 3. However, ignoring the crack initiation phase may lead to an overly pessimistic appraisal of residual strength or remaining service life.

For accurate condition assessment and service life prediction, there is a need to track the evolution of microstructural damage prior to the state where there is some detectable manifestation of deterioration. The relatively new field of continuum damage mechanics (Kachanov, 1986; Chaboche, 1988; Lemaitre, 1992) provides one possible approach, which will be explored in a later phase of this research. A second is to take advantage of the apparently close correlation of magnetic and mechanical properties of ferromagnetic materials (Jiles, et al, 1994). Ware and Shah (1995) found that magnetic hysteresis measurements during cyclic loading could be used to track the evolution of fatigue damage. A533B steel (softening material) tension specimens were cycled under both constant amplitude strain (low-cycle) and load (high-cycle) controlled conditions. Measurements of magnetic hysteresis during cycling indicated that the magnetic properties remained quite stable over $80-90$ percent of the fatigue life, but changed dramatically as macrocracks formed. 
Table 2.1 - Uniform Corrosion Parameters in Eqn 2.1

\begin{tabular}{|c|c|c|c|c|}
\hline Environment & Steel & $\mathrm{C}$ & $\alpha$ & Ref. \\
\hline Rural & Carbon & 34 & 0.65 & Komp (1987) \\
\hline Urban & Carbon & 80 & 0.59 & " \\
\hline Marine & Carbon & 71 & 0.79 & $"$ \\
\hline Rural & Weathering & 33 & 0.50 & $"$ \\
\hline Urban & Weathering & 51 & 0.57 & $"$ \\
\hline Marine & Weathering & 40 & 0.56 & " \\
\hline Industrial & Carbon & 51 & 0.42 & Structural (1989) \\
\hline Industrial & USS Cor-Ten & 25 & 0.17 & " \\
\hline Ocean $(5500 \mathrm{ft})$ & A36 & 70 & 0.40 & Structural (1989) \\
\hline Ocean $(5500 \mathrm{ft})$ & A36 & 138 & 0.25 & $"$ \\
\hline Ocean (surface) & Carbon & 229 & 0.69 & $"$ \\
\hline Ocean (surface) & Carbon & 200 & 0.62 & $"$ \\
\hline Ocean (surface) & Carbon & 144 & 0.79 & $"$ \\
\hline $40 C-80 C$ & AISI 316 Stainless & 39 & 0.36 & Porter, et al (1994) \\
\hline
\end{tabular}

Table 2.2 - Pitting Corrosion Parameters in Eqn 2.1

\begin{tabular}{lllll}
\hline Environment & Steel & C & $\alpha$ & Ref. \\
\hline \hline $40 \mathrm{C}-80 \mathrm{C}$ & AISI 316 Stainless & 1000 & 1.0 & Porter, et al (1994) \\
$\mathrm{NaHCO}_{3}$ & Carbon & 7000 & 0.42 & Sharland, et al (1991) \\
$\mathrm{NaHCO}_{3}+\mathrm{Cl}$ & Carbon & 8350 & 0.46 & Marsh, et al (1985)
\end{tabular}




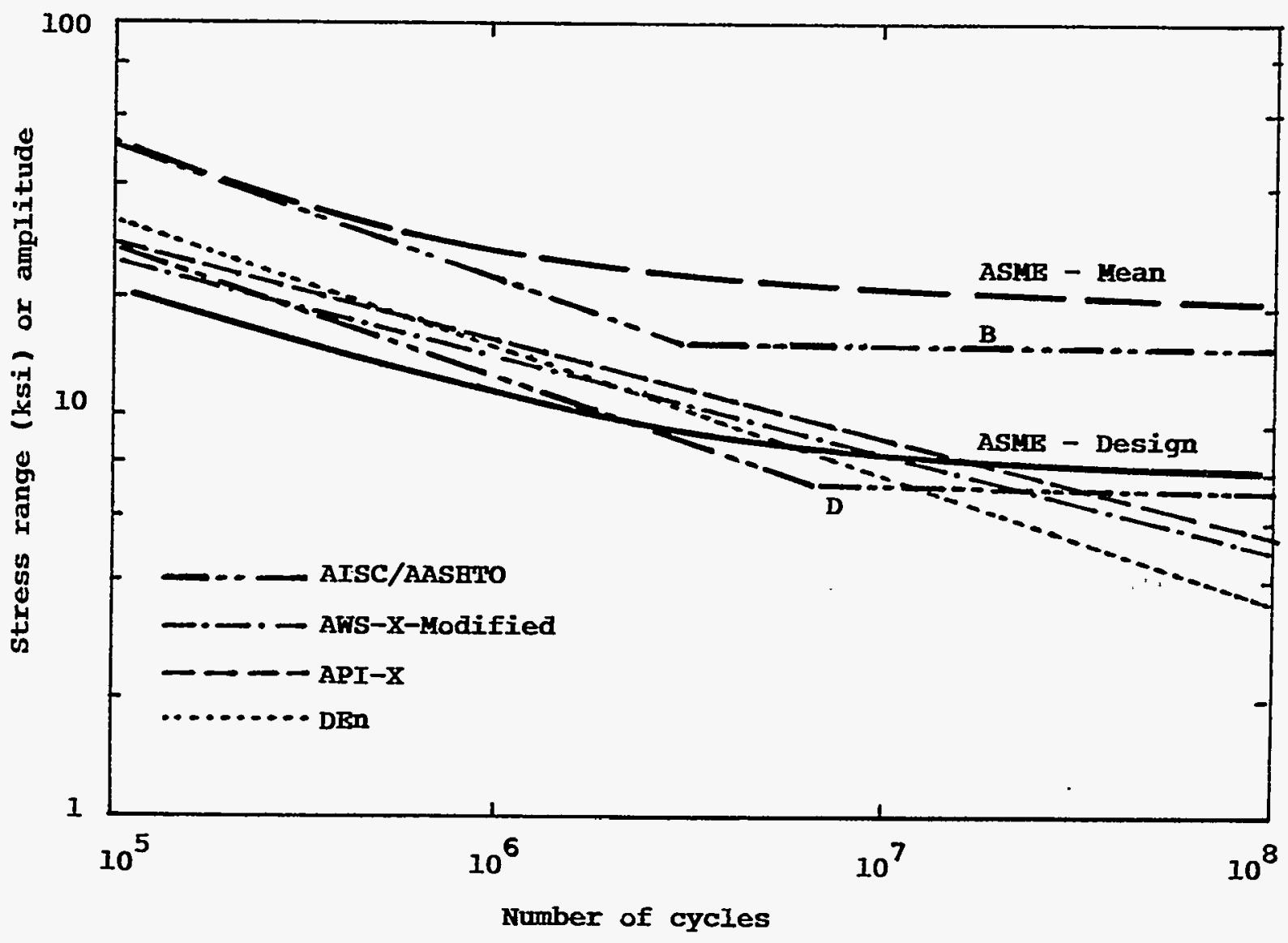

Figure 2.1 Typical S-N curves for fatigue design 


\section{NONDESTRUCTIVE EVALUATION METHODS}

Nondestructive evaluation methods (NDE) are used to examine materials or components in ways that do not impair function in service. NDE is used to (1) locate and size flaws; (2) measure geometrical characteristics, and (3) assess composition. From an operational viewpoint, such inspections are required to identify potential challenges to structural integrity in time to take remedial action. They also play an important role in structural reliability assessment, especially when combined with failure analysis techniques such as fracture mechanics. Factors that are important in a reliability-based condition assessment include probability of detection, threshold of detection and flaw size distribution, and sizing accuracy. NDE provides an opportunity to revise and update the probability models used to determine current margins of safety and to forecast future reliability and performance. An ASME Research Task Force on risk-based inspection recently has been created (ASME, 1992).

The most common NDE techniques in civil structures are Visual Inspection (VT), Eddy Current (EC), Magnetic Particle Inspection (MT), Liquid Penetrant (PT), Radiography (RT), Ultrasonic Inspection (UT) and Acoustic Emission (AE). However, no in-service inspection is perfect. NDE outputs depend on many factors, including the sensitivity of the instruments to different types of flaws, human factors such as education, training and proficiency of operators, geometry and microstructure of the component inspected, and size of flaws. Many of the NDE methods initially were developed to inspect components during relatively wellcontrolled manufacturing processes. They may be difficult to use in condition assessment and aging management, where quantification of flaw size is necessary and limitations on the sensitivity of NDE are amplified by difficult field conditions. The procedures used in service frequently are manual and time consuming. A flaw of a given size can be detected only with a certain probability; for any but the largest defects, however, there is a finite probability that the flaw escapes detection. Conversely, there is a possibility that NDE indicates a flaw when none is present (a so called false call); repair actions in such a case not only would be unnecessary but might damage the structure. Moreover, the actual flaw present may not be measured accurately by the NDE method chosen. Detection and measurement uncertainties arising from NDE must be incorporated in the reliability analysis.

Significant portions of NPP structures where damage might occur are not easily accessible to inspection. To maximize the efficiency of the inspection process, sampling plans must be devised that require only portions of the structure to be inspected, rather than the entire structure. The basic idea of such a sampling plan is to focus initial inspection on a small (critical) portion (typically 5-10 percent) of the structure. If no problems are found in this first stage, the structure as a whole is deemed acceptable until the next scheduled inspection. If flaws are located, an additional portion (say, 10-20 percent) is inspected. If no further problems are evident in this second stage, the result of the initial stage is viewed as an anomaly; if, on the other hand, problems are evident in the second stage as well, the entire structure may be inspected prior to continued service. Inspection is thus a living process. Obviously, a large portion of the structure may remain uninspected; any undetected flaws impact the time-dependent reliability.

In the following sections, common NDE methods will be assessed (Rummel, et al, 1989; Bray and Stanley, 1989), with particular attention to quantifying detection and measurement uncertainties.

\subsection{Detection of flaws}

The probability of detecting a flaw depends on the NDE method employed, the size of the flaw and the training of the operator. When NDE is performed, four outcomes are possible, as shown in the following: 


\begin{tabular}{|l|c|c|}
\hline & positive call $(\mathrm{D})$ & negative call $(\mathrm{N})$ \\
\hline Flaw $(\mathrm{F})$ & FD & FN \\
\hline No flaw $(\overline{\mathrm{F}})$ & $\overline{\mathrm{FD}}$ & $\overline{\mathrm{FN}}$ \\
\hline
\end{tabular}

Event FD denotes that a flaw exists and is detected, event FN denotes that a flaw exists but is not detected, event $\bar{F}$ depicts that no flaw exists but a flaw is indicated, and event $\overline{F N}$ indicates that no flaw exists and none is indicated. These imply that for a given flaw, there is only a certain probability of detecting it. Also, even though there is no flaw present, we might possibly have a false call.

The probability of detection (POD) expresses the probability of detecting a crack in a given size group under specified inspection conditions and procedures. Many NDE methods indicate the presence of a flaw indirectly rather than directly. The signal shown may not be the physical characteristics of interest, such as length and depth, but other control parameters corresponding to the technique used, such as a voltage for UT. As a result of surface roughness, microstructure inhomogeneities and other factors, the signal observed also includes inherent noise. Suppose that $Y$ is this observation variable. If $Y>y_{t b}$, where $y_{t h}$ is given threshold, a flaw is indicated; otherwise, the indication is assumed to be simply noise. For a crack with size a, $\operatorname{POD(a)}$ can be expressed as:

$$
\operatorname{POD}(a)=\lim _{n \rightarrow \infty} \frac{\text { number of positive calls } n_{p}}{\text { number of defects present } n}
$$

or

$$
\operatorname{POD}(a)=\int_{y_{t}}^{\infty} f\left(y_{a}\right) d y_{a}
$$

where $\operatorname{POD}(\mathrm{a})$ is the probability of detecting a crack with size a. Eqn. 3.1 expresses POD(a) in the form of a relative frequency. The denominator represents the total number of flaws with size a present in the components tested, while the numerator designates the number of the flaws indicated. Eqn. 3.2 defines $\mathrm{POD}(\mathrm{a})$ in the form of a cumulative distribution function (CDF). $\mathrm{Y}_{\mathrm{a}}$ is the signal response amplitude with respect to the flaw with size $a$. It is a random variable with probability density function $f\left(y_{a}\right)$. The above integral thus specifies the probability of detection for a given flaw size. For the special case when $a=0$, i.e., there is no existing flaw, $P O D$ (a) represents the false call probability (FCP). Varying the flaw size and plotting POD(a) gives a POD curve. Generally, POD(a) increases with increasing flaw size. Several commonly used POD models will be introduced in the following discussion.

Berens (1989) suggested that a log-logistic function is a suitable model to fit the POD data. Two mathematically equivalent forms are given:

$$
\ln \frac{\operatorname{POD}(a)}{1-\operatorname{POD}(a)}=\alpha+\beta \ln a ; \quad a>0
$$

or

$$
\operatorname{POD}(a)=\left(1+\exp \left(-\left(\frac{\pi}{\sqrt{3}}\left(\frac{\ln a-\mu}{\sigma}\right)\right)\right)\right)^{-1} ; \quad a>0
$$

where $\alpha, \beta, \mu, \sigma$ are unknown parameters which can be estimated through regression analysis. Parameter $\mu$ $=\ln \mathrm{a}_{0.5}$, where $\mathrm{a}_{0.5}$ is the median flaw size satisfying $\operatorname{POD}\left(\mathrm{a}_{0.5}\right)=0.5 ; \sigma$ is related to the steepness of the POD(a) curve, a smaller value of $\sigma$ being associated with a steeper POD(a) curve. 
The relationship between $(\alpha, \beta)$ and $(\mu, \sigma)$ is,

$$
\begin{aligned}
& \mu=-\frac{\alpha}{\beta} \\
& \sigma=\frac{\pi}{\beta \sqrt{3}}
\end{aligned}
$$

The log-logistic model is similar to a lognormal distribution.

Others have suggested that the POD curve can be given by the exponential distribution,

$$
\operatorname{POD}(a)= \begin{cases}1-\exp \left(-c\left(a-a_{t h}\right)\right) & ; a<a_{t h} \\ 0 & ; a<a_{t h}\end{cases}
$$

in which $\mathrm{a}_{\mathrm{th}}=$ minimum detectable defect and $\mathrm{c}=$ constant, both of which depend on the NDE device and its resolution (Tsai \& Wu, 1993) or, more generally, a Weibull distribution (Kennedy, et al, 1991). If NDE is perfect, every defect above the threshold of detection, $a_{t h}$, would be detected, and POD(a) would take on the appearance of a Heaviside function,

$$
\operatorname{POD}(\mathrm{a})=\mathrm{H}\left(\mathrm{a}-\mathrm{a}_{\mathrm{th}}\right)
$$

Such is not the case, of course; however, one would like $c$ to be as large as possible in order to approach this condition.

Both of the models mentioned above are consistent with the intuition that large defects almost certainly will be detected while very small defects will almost certainly be missed, assuming that the entire component is inspected. However this may not be the case. Considering that there may not be a certainty for detecting even very large defects, an alternative expression for the probability of no detection is proposed (Staat, 1993),

$$
1-\operatorname{POD}(\mathrm{a})=\mathrm{p}+(1-\mathrm{p}) \exp (-\mathrm{ca})
$$

in which $\mathrm{p}$ and $\mathrm{c}$ are parameters dependent on the NDE method. The corresponding POD is,

$$
\operatorname{POD}(a)=(1-p)(1-\exp (-c a)) ; \quad a \geq 0
$$

Note that this $\operatorname{POD}(\mathrm{a})$ is asymptotic to 1 - $\mathrm{p}$ for large values of a. There is no threshold of detection, i.e., defects larger than zero have a finite POD. A model combining the best features of both Eqn 3.7 and 3.10 would be,

$$
\operatorname{POD}(a)=(1-p)\left(1-\exp \left(-c\left(a-a_{t h}\right)\right)\right) ; a \geq a_{t h}
$$

The probability of detecting defects smaller than $\mathrm{a}_{\mathrm{th}}$ would be zero, while the probability of detecting very large defects would be 1 - p; typically $p$ would be on the order of $0.001-0.05$.

One disadvantage of the models represented by Eqns $3.7-3.11$ is that none of them incorporates the false call probability (FCP) which may occur with some NDE methods. For example, in their pipe crack detection round robin studies, Heasler et al (1990) found that the false call probability can be as high as $27 \%$. 
Taking into account this FCP, Heasler, Taylor and Doctor (1993) proposed a logistic model using a instead of In a in Berens' model:

thus,

$$
\operatorname{POD}(a)=(1+\exp (-(\alpha+\beta a)))^{-1}
$$

$$
\mathrm{FCP}=(1+\exp (-\alpha))^{-1}
$$

Generic POD curves using the above models are illustrated in Figure 3.1. Specific NDE methods are considered in the following sections.

\subsection{NDE techniques}

\subsubsection{Surface and near-surface methods}

Visual inspection (VT) is the oldest and still most widely used NDE method. In NPPs, accessible surfaces of the pressure-retaining boundary are inspected prior to each integrated leakrate test. Underwater inspections for cracks usually are performed visually and are supplemented by magnetic particle inspection after cleaning (Kishi, 1988). Visual inspection can identify regions of corrosion, or peeling or blistering of coatings that may indicate damage to the substrate. Special attention must be paid to welds and heat-affected zones of weldment.

Liquid penetrant (PT) is effective in locating surface flaws in essentially nonporous materials. The fluorescent or visible penetrant seeps into various types of minute surface openings by capillary action, giving indications of defects. The advantage of this method is that it depends neither on ferromagnetism (as does, for example magnetic particle inspection) nor on defect orientation as long as only surficial flaws are considered. The major limitation of PT is that it cannot detect subsurface flaws and can be excessively influenced by the surface roughness or porosity. Studies (Chase, 1994) of application of NDE to the detection of fatigue cracks in steel bridges have revealed that the crack length sensitivity range is $7-13 \mathrm{~mm}$ in welds and greater than $24 \mathrm{~mm}$ in joints using PT; others have reported similar thresholds of detection (approximately $9 \mathrm{~mm})$.

Figure 3.2(a) illustrates a POD curve for PT from analysis of data from 328 fatigue cracks in 118 aluminum alloy specimens (Rummel, et al 1989). There is no false call probability indicated; the authors claimed that FCP was "not reflected by the POD curve" because in their experiment, no inspections were conducted on unflawed areas. As will be seen later, the other three POD curves they obtained using Eddy Current, Ultrasonic and X-ray inspection also indicated no FCP. The minimum flaw size here is about $0.5 \mathrm{~mm}$. Using the model in Eqn. 3.4 we have $\mu \approx 0, \sigma \approx 0.3 \mathrm{~mm}$; for the model of Eqn. $3.7, \mathrm{a}_{\mathrm{th}}=0.5 \mathrm{~mm}, \mathrm{c} \approx 2 / \mathrm{mm}$.

Magnetic particle inspection (MT) is utilized to reveal surface and subsurface discontinuities in ferro-magnetic materials. When the material is magnetized, a leakage field is generated by magnetic discontinuities that lie in a direction transverse to the direction of the magnetic field. The leakage field gathers and holds some of the fine ferromagnetic particles applied over the surface. This forms an outline of the discontinuity and indicates its location, size and shape. MT is capable of detecting fine, sharp and shallow surface cracks in ferro-magnetic materials, but is not good for wide and deep defects. It cannot be used for nonferromagnetic materials. The magnetic field must be in a direction that intercepts the principal plane of discontinuity for a good result. Thin coatings of paint and other nonmagnetic coverings will adversely affect the sensitivity. MT is effective in detecting surficial defects in excess of about $6 \mathrm{~mm}(1 / 4 \mathrm{inch})$ long. The probability of detection is strongly dependent on field conditions.

Figure 3.2(b) illustrates a typical POD curve for MT obtained by Packman et al (1969). The material was AISI 4330 vanadium modified heat treated steel. 


\subsubsection{Ultrasonic inspection (UT)}

UT is used to detect both surface and internal discontinuities in materials and can also be used to identify areas of thinning due to corrosion. Beams of high frequency sound waves introduced into the material attenuate due to wave scattering and are partially or completely reflected at interfaces. The reflected beam is displayed and analyzed to define the presence and location of defects such as cracks or voids. UT can also be used to measure thickness and extent of corrosion by monitoring the transit time of a sound wave through the component or the attenuation of the energy. UT can be performed under water. The principal advantages of UT are its portability and superior penetrating power and volumetric scanning ability which allow the detection of deep flaws. Studies (Chase, 1994) show that its sensitivity to crack length is the highest among the commonly used NDE techniques (detection thresholds between 3-7 mm in welds and 7-13 mm in joints), and its complexity and operator dependence are moderate. Its disadvantage is that defects in parts that have rough or irregular surfaces, or are very small, thin or non homogeneous are difficult to detect.

Research has been in progress for several years to determine reliability of in-service ultrasonic inspection and its capability for flaw sizing. The aim of this research is to establish reliability of the inspection process for pressure vessels and primary coolant piping systems in NPPs (Doctor, et al, 1993). Techniques other than UT are not being considered. Data are being obtained from an international round-robin test of ultrasonic inspection capabilities involving teams from the United States, several European countries and Japan. Data were gathered during an exercise called PISC II (Programme for the Inspection of Steel Components, Phase 2). Human factors are being incorporated in the study. It was found that flaw length is the best control parameter in determining probability of detection curves. Results of this activity are being interfaced with an ASME task force on reliability-based in-service inspection (ASME, 1992). Typical POD curves developed as part of this study (Heasler, et al, 1993) are illustrated in Figure 3.3(a); points identified as $\mathrm{H}$ or $\mathrm{L}$ indicate potentially anomalous data. As a comparison, the curve obtained by Rummel, et al (1989) is shown in Figure 3.3(b). Using the POD models in Eqn. 3.10 to fit the data in Figure 3.3(a), we have $p=$ $0.005, c=0.1134 / \mathrm{mm}$ (Kennedy, et al, 1991; Staat, 1993); using Eqn. 3.12, $\alpha \approx-1.73, \beta \approx 1.5$. A curve similar to Eqn. 3.7, in which $c=0.113 / \mathrm{mm}$, was recommended by Tsai and Wu (1993). It is noted that FCP is significant for UT because it mainly deals with internal flaws and is not visually-assisted.

\subsubsection{Eddy current (EC)}

EC is effective in detecting defects at or within a few millimeters of the surface. It is based on the principle of electromagnetic induction. Taking a pipe as an example, a current is created by encircling the pipeline with induction coils, The presence of a crack in the pipe impedes the current flow and changes its direction, causing changes in the associated electromagnetic field which can be monitored. Thus surface discontinuities having a combination of predominantly longitudinal and radial dimensional components can readily be detected.

A majority of surface discontinuities can be detected by EC with high speed and low cost. If a coating is present, it need not be removed. However, the sensitivity of this method to defects beneath the surface is decreased. Also, laminar defects may not alter the flow enough to be detected. Defects less than $6 \mathrm{~mm}(1 / 4$ in) at the toe of a weld reportedly cannot be detected by EC (Shah, et al, 1994), nor can defects more than about $13 \mathrm{~mm}(1 / 2 \mathrm{in})$ below the surface. The sensitivity ranges of fatigue cracks in steel bridges are $7-13 \mathrm{~mm}$ in welds and greater than $25 \mathrm{~mm}$ in joints (Chase, 1994), comparable to that of PT.

In one study (Bowen, et al, 1989), research was conducted on the reliability of eddy-current inspection techniques to detect and size flaws in steam generator tubes. Human factors also were taken into account in this study, and performance of several inspection teams was considered. Typical POD data collected in this study are shown in Figure 3.4(a); the lines correspond to lower bound and median trends. As a comparison, 
the curve obtained by Rummel, et al (1989) is shown in Figure 3.4(b): Using the model in Eqn. 3.7 to fit the data in Figure 3.4(a), we have

$$
\operatorname{POD}(a)=1-\exp (-3.5(a-0.10))
$$

in which a=true flaw depth, expressed as a fraction of plate or weld thickness. Recognizing that the model does not incorporate FCP, which appears to be apparent in the data, one might use the model in Eqn 3.12 with $\alpha \propto-2.94$ and $\beta \propto 9$.

\subsubsection{Acoustic emission (AE)}

Sudden movement in stressed materials produces acoustic stress waves. The stress waves can be detected on the surface of the structure by one or more piezoelectric transducers. One source of AE is defectrelated active deformation processes such as fatigue crack growth. Thus, AE offers the possibility of monitoring growing defects during service. Research (Yeh, Enneking and Tsai, 1994) has been conducted to relate AE energy counts to stress intensity factor and strain energy release rate. However, difficulties still remain in using acoustic transducers to locate or size growing defects accurately due to the noise resulted from various sources, and the $\mathrm{AE}$ is better used in conjunction with other flaw detection methods. Efforts have been made (Ghorbanpoor, 1994) to improve signal discrimination techniques for $\mathrm{AE}$ evaluation of steel bridges. The sensitivity ranges for fatigue cracks in steel bridges are $7-13 \mathrm{~mm}$ in welds and $13-25 \mathrm{~mm}$ in joints, respectively. No further information on detection probability for AE could be located. The technique is still relatively new in its application to civil structures.

\subsubsection{Radiography (RT)}

RT methods are based on the differences in absorption by different portions of a component of penetrating radiation, such as X-ray or $\gamma$-ray. The images produced can be analyzed to locate flaws. Planar defects cannot be detected unless their principal plane is essentially parallel to the radiation beam. Tight cracks are difficult to detect regardless of orientation. In contrast to the other methods above, access to both sides of the component is required. Safety protocols also must be followed. RT is relatively expensive. Figure 3.5 illustrates a typical POD curve for X-ray inspection obtained by Rummel, et al (1989) by gathering data from 328 fatigue cracks in 118 aluminum alloy specimens. Using the POD model in Eqn 3.7, we have $\mathrm{a}_{\mathrm{hh}} \propto 0.51$ $\mathrm{mm}, \mathrm{c} \approx 0.35 / \mathrm{mm}$.

\subsection{Flaw measurement errors}

Error in sizing is also an important issue because the defect size identified is greatly affected by many factors such as education of operators, sensitivity of equipment, procedures conducted and material imperfections. For example UT can be used to locate areas of corrosion in inaccessible regions, but may not correctly identify the extent of corrosion penetration if the surface roughness due to corrosion is high and wave scattering occurs as a result. Errors in measuring thickness ultrasonically for several commercially available UT gauges (summarized in Figure 3.13 of Shah, et al, (1994)) and in sizing can be as high as 40 percent for a surface roughness of $0.2 \mathrm{~mm}$ RMS. The pipe inspection round robin conducted by Heasler, et al (1990) also showed that sizing performance was not very good. Slopes of regressions of true sizes on measured sizes from UT sometimes are close to 1 and other times deviate from 1 ; variability is high, with an average standard error of 20 percent. Other techniques such as EC and RT have similar sizing errors. Error in sizing by PT and MT seems to be less of a problem mainly because these techniques deal with surficial flaws where inspections are visually assisted. Figure 3.6 illustrates the data scattering difference between PT and X-ray inspection.

After a flaw has been detected, it is necessary to estimate the true flaw size, a, from the measured size, $a_{m}$. Usually, we are interested in $P\left(A<y \mid A_{m}=c\right)$, the probability that true flaw size, $A$, is less than y under 
the condition that measured size, $A_{m}$, equals $c$. Suppose that $A$ and $A_{m}$ are discrete random variables and $D$ denotes the event of detection; we have,

$$
\begin{aligned}
P\left(A<y \mid A_{m}=c\right) & =\frac{P\left(A<y \cap A_{m}=c\right)}{P\left(A_{m}=c\right)} \\
& =\frac{P\left(A<y \cap A_{m}=c \cap D\right)}{P\left(A_{m}=c\right)} \\
P\left(A<y \cap A_{m}=c \cap D\right) & =\sum_{i=1}^{n} P\left(A=a_{i} \cap A_{m}=c \cap D\right) \\
& =\sum_{i=1}^{n} P\left(A_{m}=c \mid\left(A=a_{i} \cap D\right)\right) P\left(A=a_{i} \cap D\right) \\
& =\sum_{i=1}^{n} P\left(A_{m}=c \mid\left(A=a_{i} \cap D\right)\right) P O D(a) P\left(A=a_{i}\right) \\
& =\sum_{i=1}^{\infty} P\left(A=a_{i} \cap A_{m}=c \cap D\right) \\
& =\sum_{i=1}^{-} P\left(A_{m}=c \mid\left(A=a_{i} \cap D\right)\right) P O D\left(a_{i}\right) P\left(A=a_{i}\right)
\end{aligned}
$$

Under the condition of detection, regression of measured size on true size is needed. Studies (Heasler et al, 1993) of various regression models reveal two commonly used relations,

or

$$
\log a_{m}=\beta_{1}+\beta_{2} \log a+\epsilon
$$

$$
a_{m}=\beta_{1}+\beta_{2} a+\epsilon
$$

where $\epsilon$ is a random variable representing replicate experimental errors with respect to $\log a_{m}$ or $a_{m}$. In one study, it was assumed that $\epsilon$ was uniformly distributed within the sensitivity limits of the NDE method (Kennedy, et al, 1991); such an assumption is difficult to justify from error analysis. Since error often arises from a series of independent factors, $\epsilon$ is assumed to be approximately normally distributed with standard deviation varying with different procedure types and operators.

Eqn. 3.22 employs a log transformation of the data, which can stabilize the errors. On the other hand this implies errors are proportional to flaw size with zero error for zero flaw size, which generally is not the case. While Eqn 3.23 implies that the error doesn't change with size, negative values in measured size may occur if the standard deviation of $\epsilon$ is too large. To study which model is better, Heasler et al (1993) calculated the standard deviation as a function of crack size by analyzing PISC-II data. They found that there is a modest increase in standard deviation with flaw size but that the standard deviation does not approach zero as the true size approaches zero. This indicates that Eqn 3.22 is not valid for small flaw sizes. Since Eqn. 3.23 is not sensitive to modest departures from the constant variance assumption, it was chosen for the sizing analysis for PISC-II data. In their earlier studies, Heasler et al (1990), also incorporated the effect of resolution limit denoting the smallest flaw that can be sized. The resolution limit is dependent on the technique used; for example, it is determined by the wavelength in the component when UT is applied. A linear model without any regard to inherent resolution limit in sizing cracks is not accurate for small defects. Heasler et al (1990) showed that employing two piecewise linear models, shown in Figure 3.7, leads to more realistic results. 
It should be noted that in the present study, detection errors and measurement errors are treated separately. The former are involved in POD, and the latter are handled by regression models. In other words, the regression of measured flaw size on actual flaw size is constructed using data sets in which detected and measured flaw size are paired, i.e., under the condition of detection.

\subsection{Summary}

Nondestructive evaluation plays a key role in reliability-based condition assessment and service life prediction. The inspection plan should consider component importance, redundancy, repetitive use (correlated defects), and prior history of performance (Banon et al, 1994a). Successful inspection requires: accessibility (and should not require extensive shutdown), a safe observation environment (no personal danger), flaw detection capability, accurate interpretation, small observation error, and competent performance from the inspector/operator (Meister, 1982). To gain the maximum useful information for safety margin evaluation and reliability-based updating, the NDE method or methods selected should be characterized by a POD curve with a low FCP, low detection threshold and high slope (Davidson, 1973, Rodrigues and Provan, 1989).

Despite advances in instrumentation, planning and interpretation will continue to depend on a considerable degree on human experience and judgement. Proper training and continuing education of inspectors is essential.

The values given in Sections 3.2 and 3.3 on flaw detection probability and measurement error are based on judgement from a review of the existing literature, and should be used with extreme caution in any time-dependent reliability analysis or probability-based condition assessment. Much of the data (e.g., Rummel, et al, 1989) were obtained under carefully controlled laboratory conditions. Very little quantitative data representative of NDE capabilities in more realistic but difficult field conditions could be located in this review. However, it is reasonable to conclude that the probability of detection and the ability to measure flaws accurately almost certainly would be much less favorable than what is illustrated in Figures 3.2 through 3.7. It is hoped that such data will be forthcoming later from other tasks of the Steel Containments and Liners Program. 


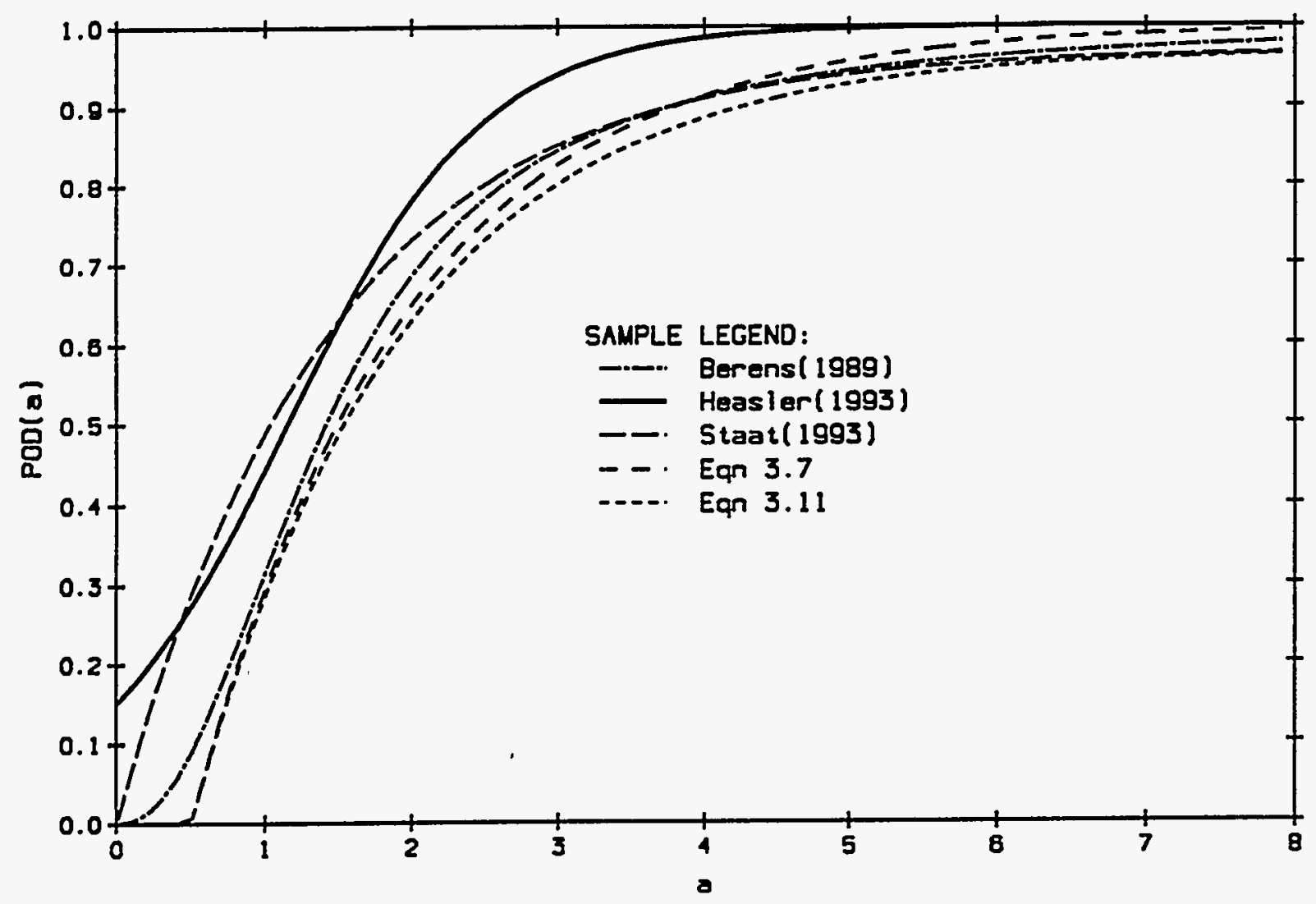

Figure 3.1 - POD curves for different models 


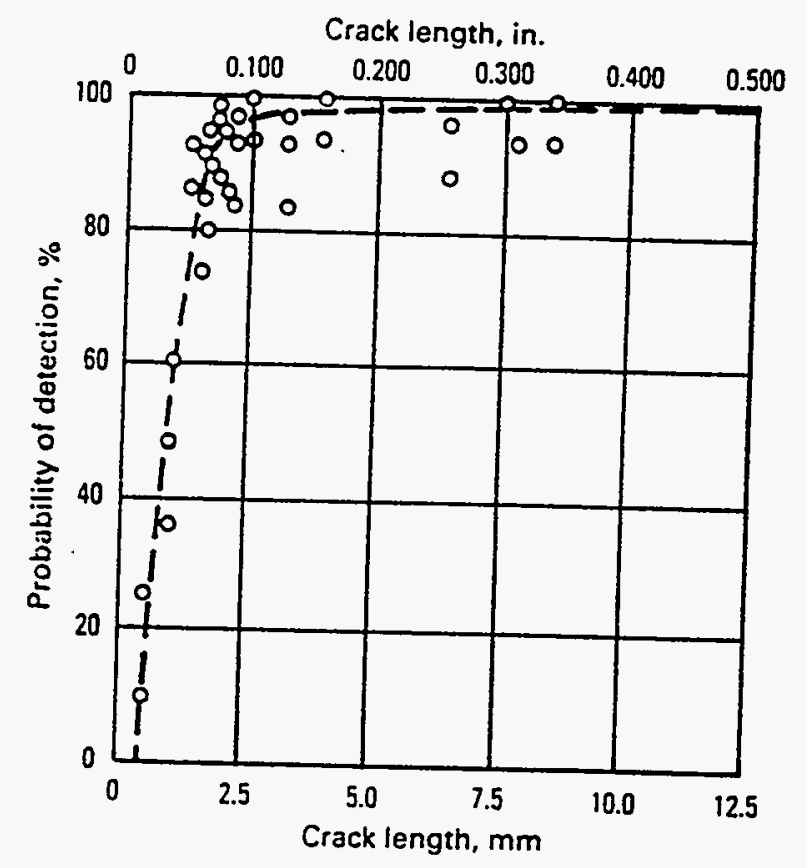

(a) - POD for PT (Rummel, et al, 1989; reprinted with permission from ASM International)

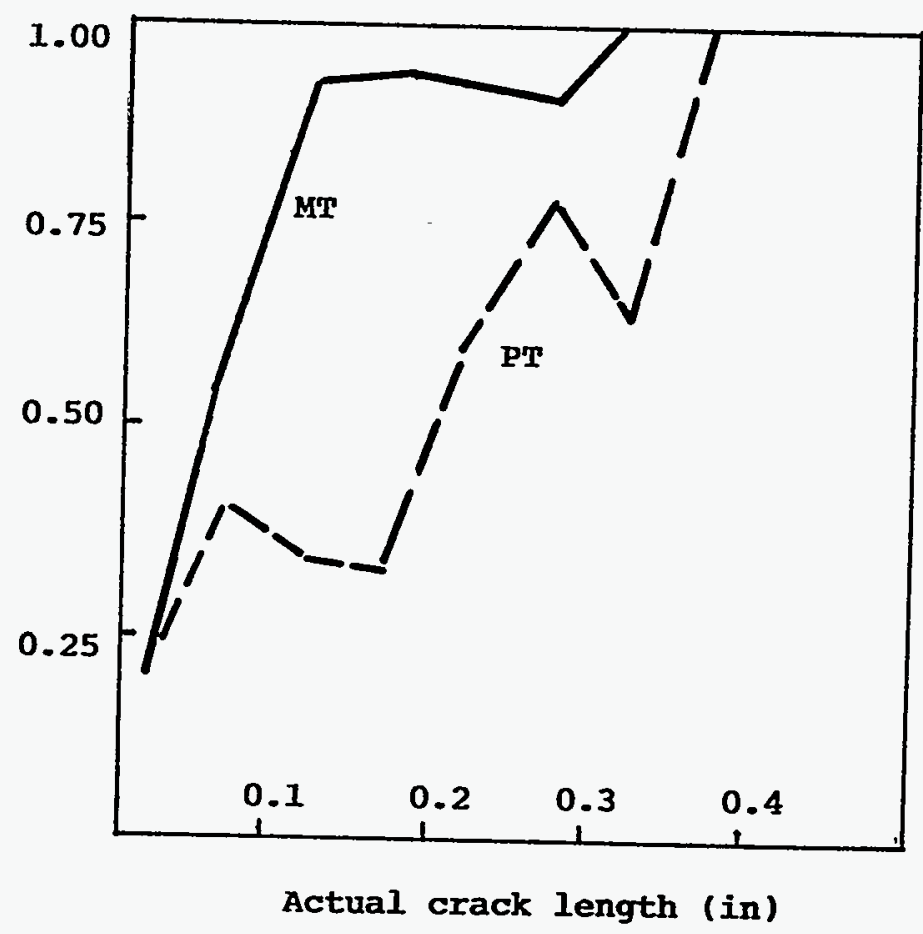

(b) - POD curve for MT and PT (after Packman, et al, 1969)

Figure 3.2 - Probability of detection - PT and MT 


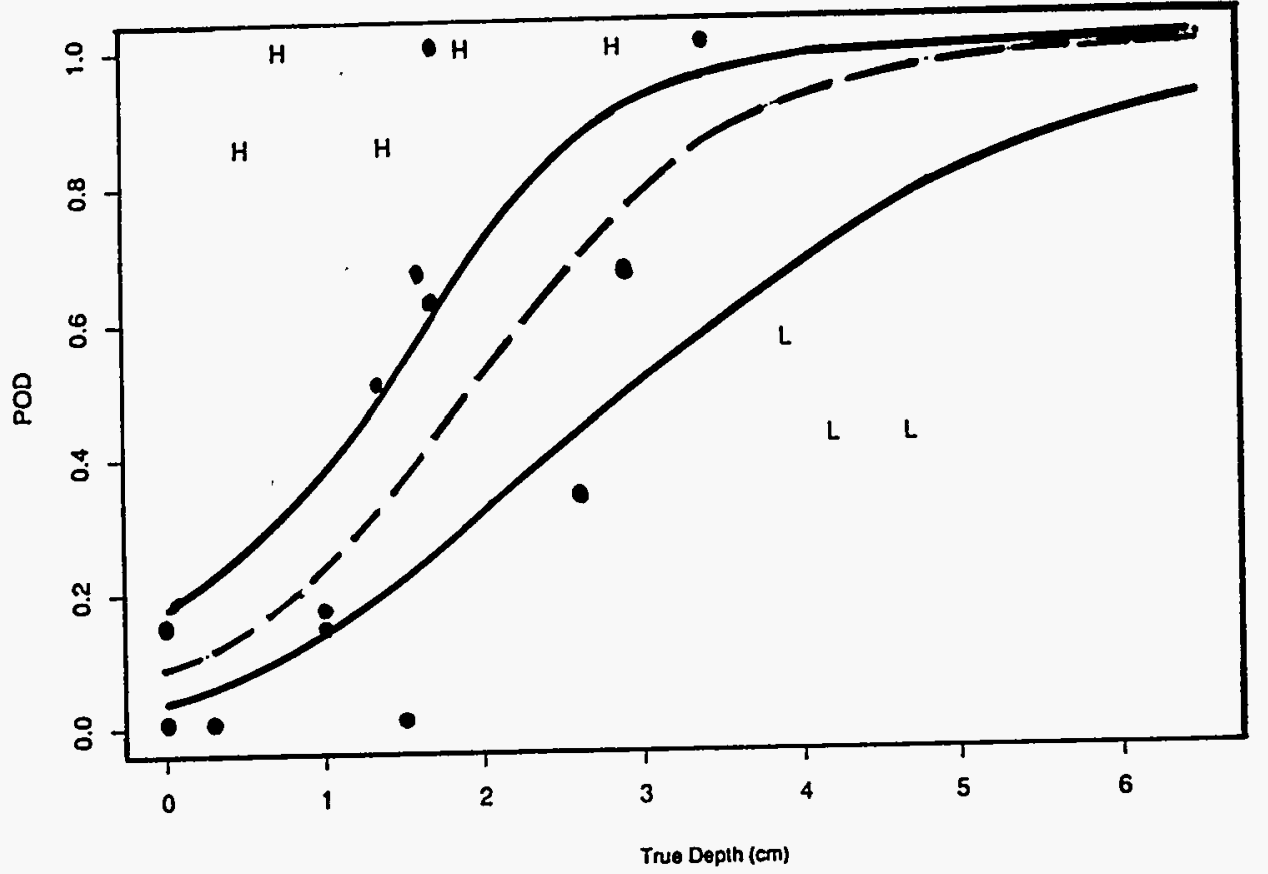

(a) - POD for UT (Heasler, et al, 1993)

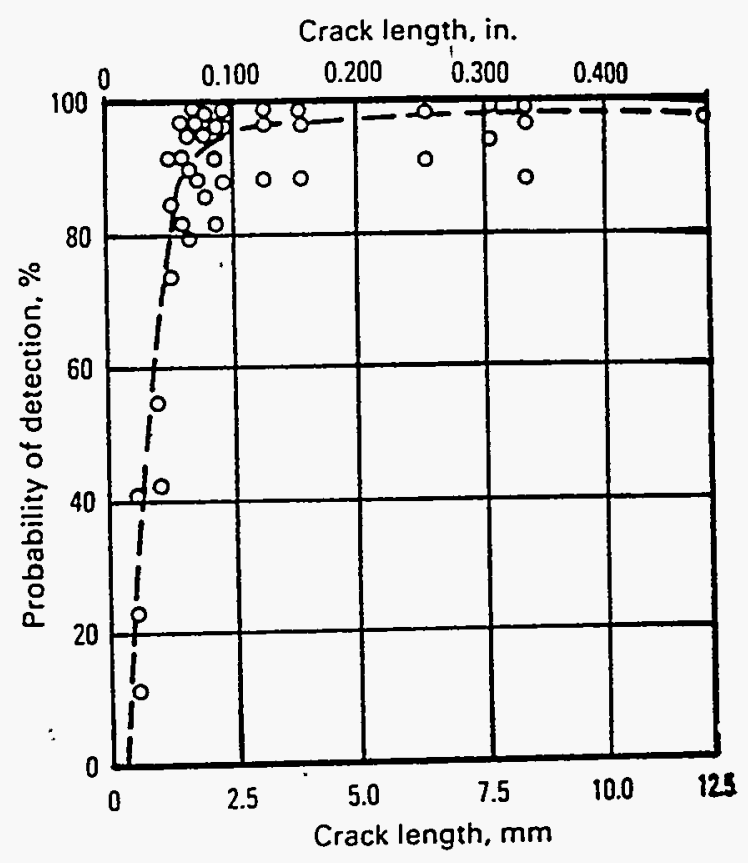

(b) - POD for UT (Rummel, et al, 1989; reprinted with permission from ASM International)

Figure 3.3 - Probability of detection - UT 


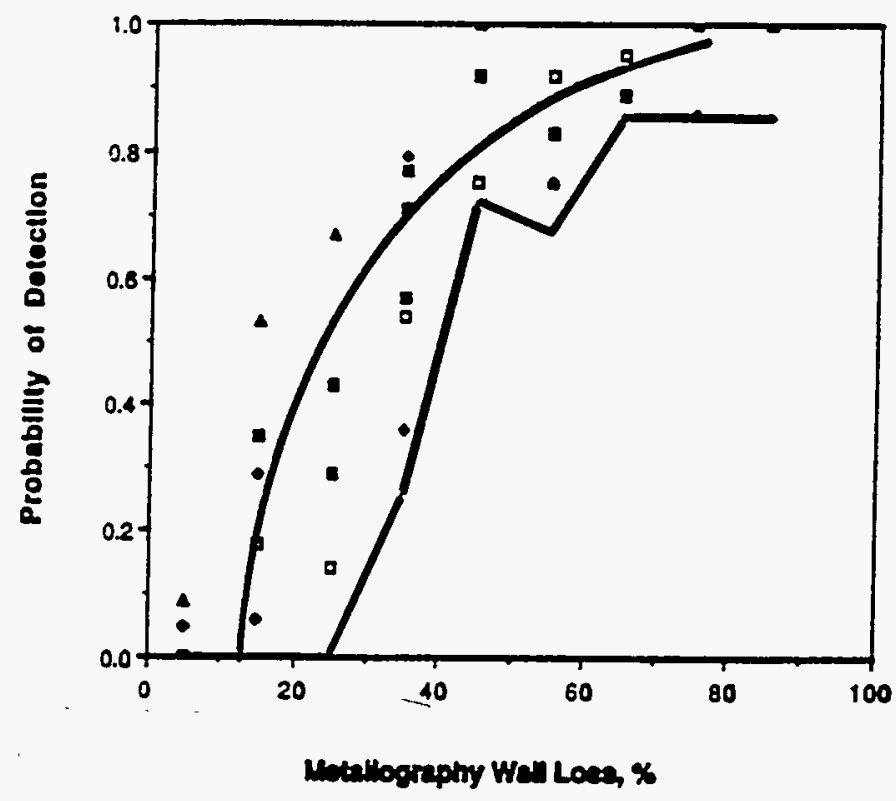

(a) - POD for EC (Bowen, et al, 1989)

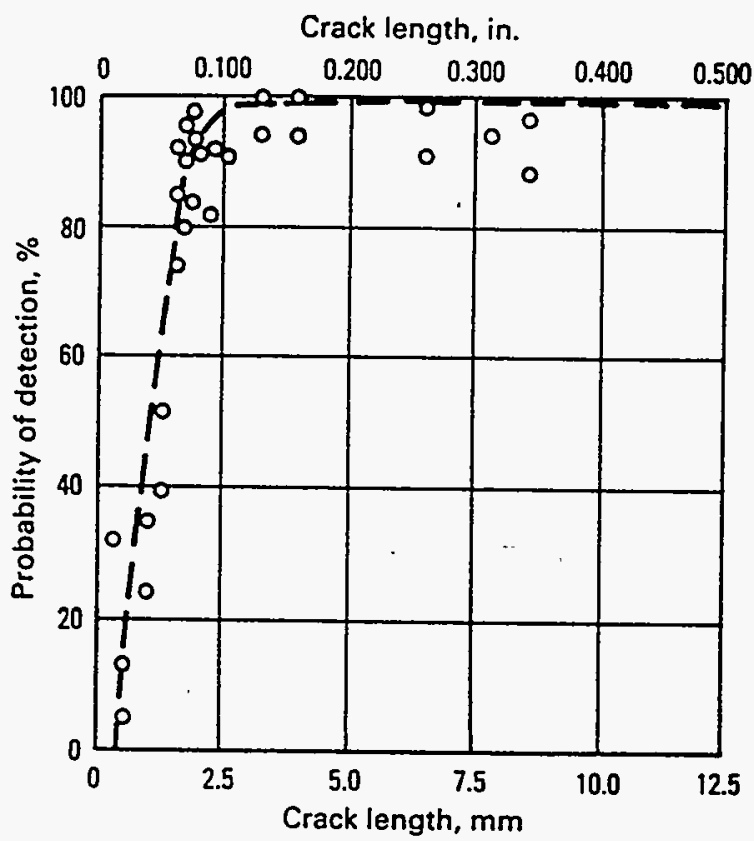

(b) POD for EC (Rummel, et al, 1989; reprinted with permission from ASM International)

Figure 3.4 Probability of detection - EC 


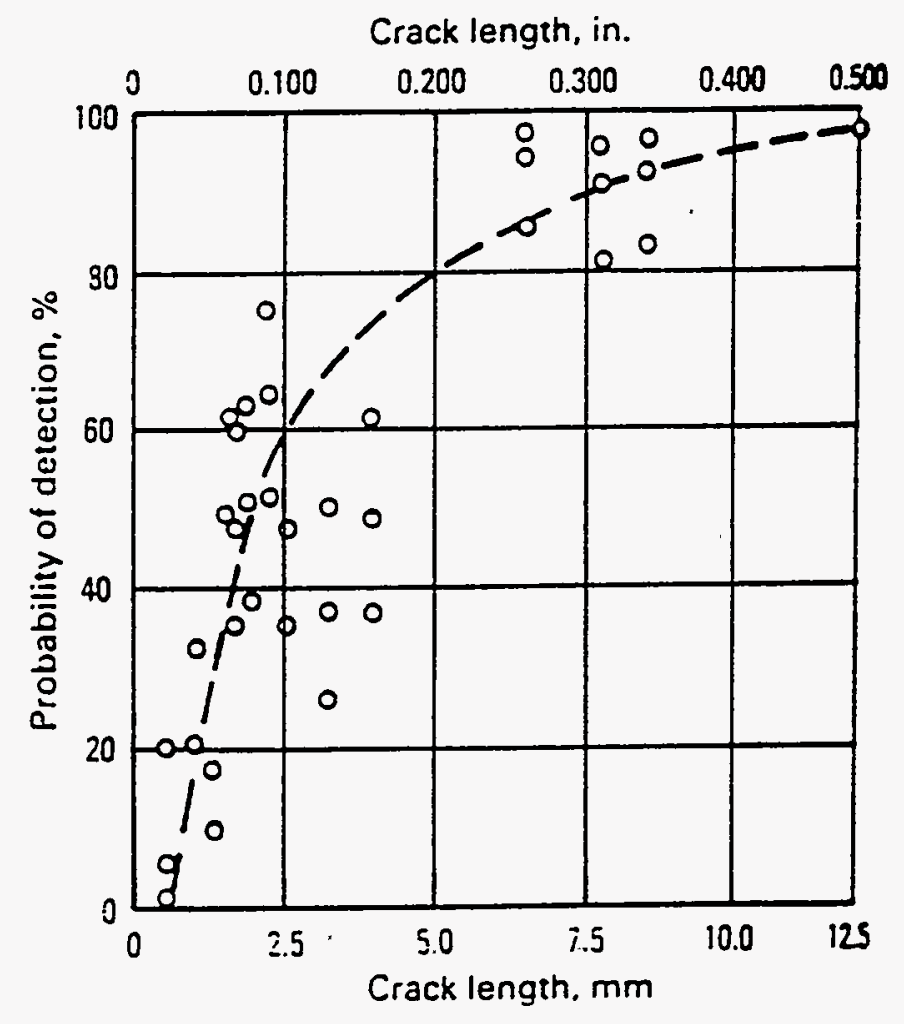

Figure 3.5 Probability of detection - RT (Rummel et al, 1989; reprinted with permission from ASM International) 


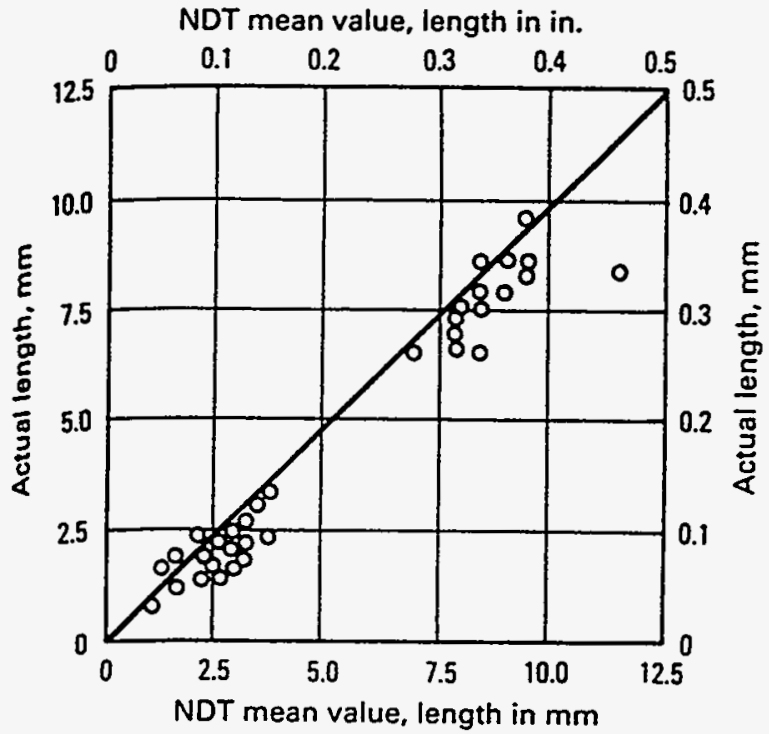

(a) PT

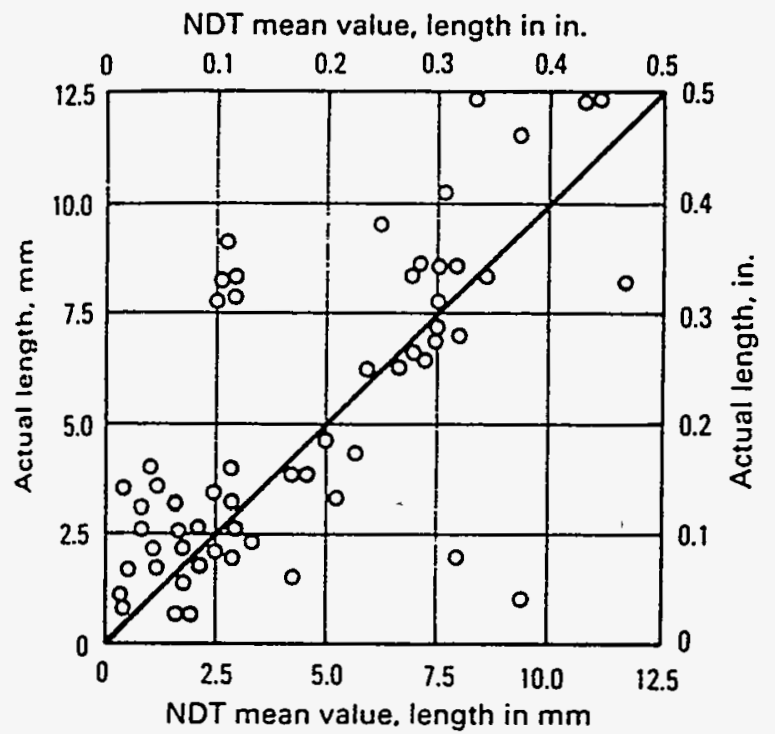

(b) RT

Figure 3.6 Actual vs measured crack length for PT and RT (Rummel et al, 1989; reprinted with permission from ASM International) 


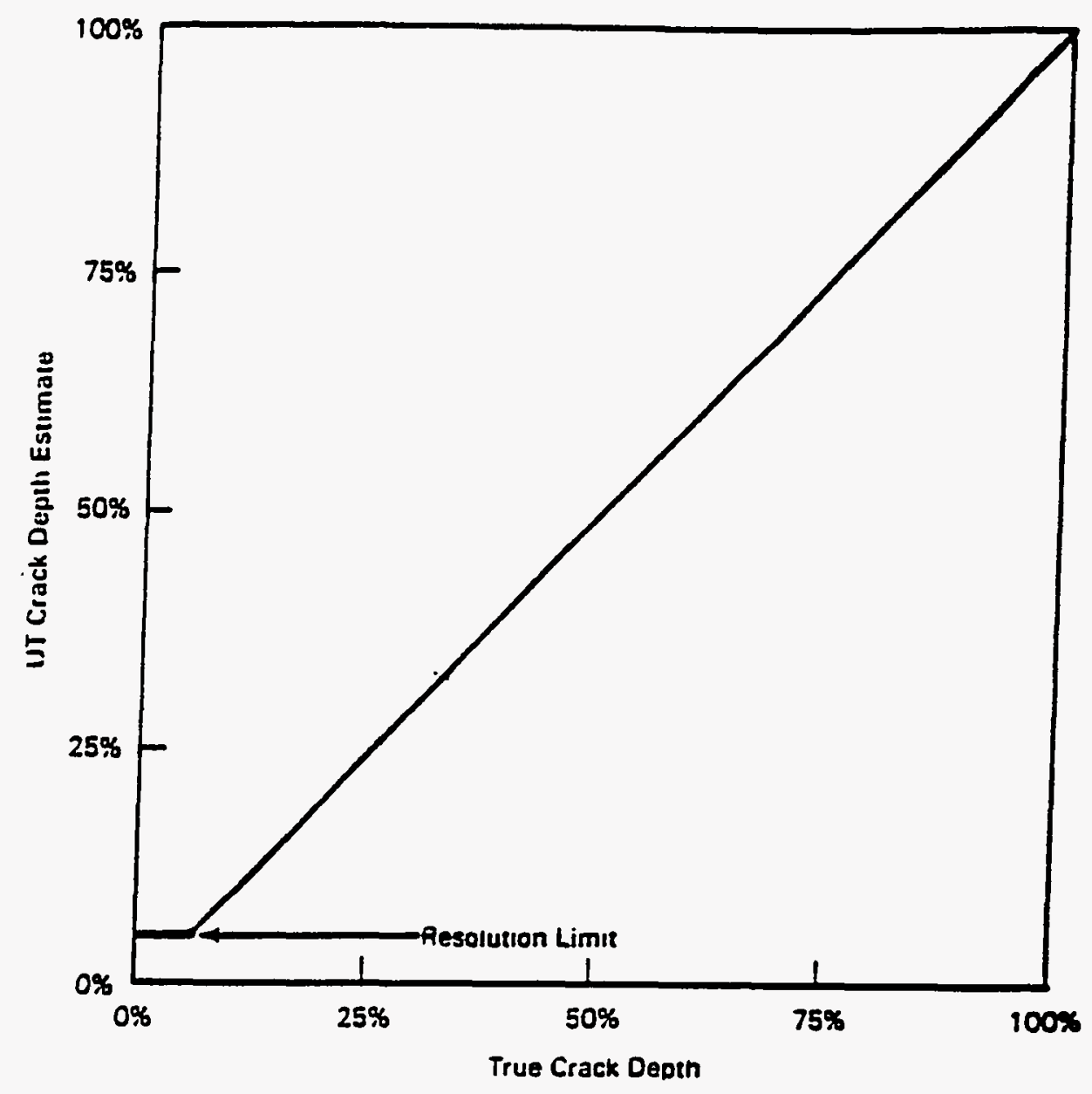

Figure 3.7 Actual vs measured crack depth including resolution limit.

Source: $\quad$ P. G. Heasier et al., "Ultrasonic Inspection Reliability for Intergranular Stress Corrosion Cracks: A Round Robin Study of the Effects of Personnel, Procedures, Equipment and Crack Characteristics," NUREG/CR-4908, Pacific Northwest Laboratory, Richland, Washington, 1990; reprinted with permission from the authors. 
\begin{tabular}{|} 
\\
\\
\\
\\
\\
\end{tabular} 


\section{TIME-DEPENDENT RELIABILITY ANALYSIS}

The evaluation of steel structures for continued service should provide quantitative evidence that their strength is sufficient to withstand future demands within the proposed service period with a level of reliability sufficient for public safety. Structural loads, engineering material properties, and strength degradation mechanisms are random in nature. Time-dependent reliability analysis methods provide the framework for dealing with uncertainties in performing condition assessments of existing and aging structures and for determining whether in-service inspection and maintenance is required to maintain their performance at the desired level. Uncertainties that complicate the evaluation of aging effects arise from a number of sources: (1) inherent randomness in structural loads, initial strength, and degradation mechanisms; (2) lack of in-service measurements and records; (3) limitations in available models for quantifying time-dependent material changes and their contribution to containment strength; (4) inadequacies in nondestructive evaluation; and (5) shortcomings in existing methods to account for repair.

\subsection{Probabilistic models of loads}

Structural loads occur randomly in time and are random in their intensity. Structural load models and descriptive load statistics have been gathered in previous research to develop probability-based limit states design and condition assessment procedures for NPP structures (Hwang, et al, 1987; Ellingwood and Mori, 1993).

Discrete load models. The duration of structural loads that arise from rare operating or environmental events, such as accidental impact, earthquakes and tornadoes, is short and such events occupy a negligible fraction of the service life of a structure. Such loads can be modeled as a sequence of short-duration load pulses occurring randomly in time. One of the simplest pulse process models is illustrated by the sample function in Figure 4.1a. The occurrence in time of the loads (impulses) is described by a Poisson process, with mean (stationary) rate of occurrence, $\lambda$, random intensity $S_{j}$ and duration $\tau$ (Pearce and Wen, 1985). The number of events, $N(t)$, to occur during service life, $t$, is described by the probability mass function,

$$
\begin{aligned}
P[N(t)=n] & =(\lambda t)^{n} \exp (-\lambda t) / n ! \\
n & =0,1,2, \ldots
\end{aligned}
$$

The intensity of each load is a random variable, described by cumulative distribution function (CDF) $F_{i}(x)$. One can generalize this process to one in which the load process is intermittent (Figure 4.1b) and the duration of each load pulse has an exponential distribution,

$$
F_{T_{d}}(t)=1-\exp [-t / \tau] ; t \geq 0
$$

in which $\tau=$ average duration of the load pulse. The probability that the load process is nonzero at any arbitrary time is $p=\lambda \tau$.

Continuous load models. Loads due to normal facility operation or climatic variations can be modeled by continuous load processes. A Poisson process with rate $\lambda$ may be used to model changes in load intensity if the loads are relatively constant for extended periods of time, as illustrated by the sample function in Figure 4.1c. Here, the duration of each load is exponential, with average duration $\tau=1 / \lambda$. Finally, loads that fluctuate with sufficient rapidity in time that they cannot be modeled by a sequence of discrete pulses can be modeled as continuously parametered stochastic processes, a sample function of which is shown in Figure 4.1d. 
Many of the loads for which nuclear power plant structures are designed can be modeled by such processes (Ellingwood, 1983; Hwang, et al, 1987). A summary of load statistics obtained from prior research is presented in Table 4.1. The operating load statistics were obtained from a consensus estimation survey (Delphi) of NPP loads (Hwang, et al, 1983). The subset of normal operating loads presented - dead, pressure, pipe reaction, restraint of thermal expansion - are typical for a variety of NPPs. The load statistics presented in Table 4.1 are believed to be sufficient for developing and testing reliability-based condition assessment methods for NPPs. However, it is known that many operating loads are plant-dependent; moreover, environmental loads may well depend on the plant site. When plant-specific load statistics are available from in-service monitoring programs or site-specific hazard analyses, they should be used in lieu of those in Table 4.1.

\subsection{Probabilistic models of resistance}

\subsubsection{Initial resistance}

The properties of steel that are required in reliability analysis of steel structures include yield strength, tensile strength, Young's modulus of elasticity, and Poisson's ratio. The existing literature on this subject for common grades of structural steel was reviewed in depth as part of the effort to develop load and resistance factor design procedures (Galambos and Ravindra, 1978; LRFD, 1993). These data are summarized in the top portion of Table 4.2. A number of ASTM designations and grades of steel are represented in these data, but they all were construction grades and are designated simply as "carbon." A lognormal CDF

$$
\mathrm{F}_{\mathrm{X}}(x)=\Phi\left(\frac{\ln (x / m)}{\beta}\right)
$$

in which $m$ and $\beta=$ median and logarithmic standard deviation of random variable, $X$, and $\Phi()=C D F$ of a standard normal variate fits all data in Table 4.2 reasonably well.

Additional data were located on the strength of various grades of carbon steel plate used as containments or liners in NPPs. Statistical data for yield and ultimate tensile strengths for specific designations of plate are presented in the lower part of Table 4.2 (Ellingwood and Hwang, 1985). In comparison with the grades of carbon steel used for rolled shapes and plates that are common to civil construction, the mean strengths are somewhat more conservative with respect to the nominal strengths while the coefficients of variation (COV) are smaller, indicating a higher standard of quality control in fabrication. There is a tendency in these data for the mean strength to decrease with increasing plate thickness, a tendency that has been observed elsewhere.

The resistance of a steel component depends on other factors besides the material strength. A simple model of resistance to a particular limit state is given by (Galambos and Ravindra, 1978),

$$
R=R_{n} M F P
$$

in which $R_{n}=$ nominal strength computed using material strengths, dimensions and analytical procedures prescribed by the code; $M=$ material factor; $F=$ fabrication factor; and $P=$ professional factor. $M, F$, and $P$ are random variables that, as a group, model the different sources of uncertainty in the resistance. To take a specific example, one might model flexural strength as,

$$
\mathrm{R}=\mathrm{B} \mathrm{F}_{\mathrm{y}} \mathrm{Z}_{\mathrm{x}}
$$

in which $F_{y}=$ random yield strength (see Table 4.2), $Z_{x}=$ plastic section modulus, and $B=$ bias factor. If the code were to require the use of elastic analysis, the nominal strength would be, 


$$
R_{n}=F_{y n} S_{x n}
$$

in which $\mathrm{F}_{\mathrm{yn}}=$ specified yield strength and $\mathrm{S}_{\mathrm{xn}}=$ handbook elastic section modulus. With the notation in Eqs 4.4 and 4.5 , the random resistance would be,

$$
R=R_{n}\left(F_{y} / F_{y n}\right)\left(Z_{x} / Z_{x n}\right)\left(B Z_{x n} / S_{x n}\right)
$$

in which, using the notation in Eqn 4.3,

$$
\begin{aligned}
& M=F_{y} / F_{y n} \\
& F=Z_{x} / Z_{x n} \\
& P=B Z_{x n} / S_{x n}
\end{aligned}
$$

With a good quality control program, the mean of $F$ is typically close to 1.0 , and its COV is 0.05 or less. The mean and $\mathrm{COV}$ of $\mathrm{P}$ depend on the fundamental assumptions underlying the analysis - use of simple flexural theory, neglect of strain hardening, etc. - and its rigor in modeling the behavior of interest. Since such assumptions usually are on the conservative side, the mean of $\mathrm{P}$ is usually greater than 1.0 , while the COV typically is on the order of $0.05-0.10$.

\subsubsection{Time-dependent deterioration in resistance}

Reliability assessments of existing steel structures that may age in time require time-dependent statistical models and descriptions of the structural resistance. Since aging has not been considered previously in probability-based design work, the available resistance statistics in Table 4.2 apply to new construction. In the absence of full-scale monitoring of structural performance, time-dependent resistance must be obtained from mathematical models of degradation mechanisms described in Section 2, along with a knowledge of the initial resistance.

The structural resistance is modeled as a time-dependent function (Ellingwood and Mori, 1993),

$$
R(t)=R_{0} G(t)
$$

in which $R_{0}^{\prime}=R(0)$, the initial resistance, and $G(t)$ is a time-dependent degradation function defining the fraction of initial strength remaining at time, $t$. Due to uncertainties in the structural impact on damage initiation and growth from aggressive environmental attack, $G(t)$ for a steel component will be a non-increasing random process unless there is some intervention in the form of in-service replacement or repair.

Conceptually, a degradation function for predicting time-dependent resistance can be associated with each degradation mechanism. In the case of corrosion, for example, it has been shown that severely corroded material has virtually no strength, whereas uncorroded material retains its original strength properties. The reduction in structural strength from corrosion comes primarily from loss of section, but also is affected by stress or strain concentrations that arise from general roughness of the corroded surface. Tests of simple tension specimens that have been uniformly corroded prior to testing have shown that their tensile strengths based on nominal area (in units of force) are proportional to the loss of section due to corrosion; however, their strains at fracture are reduced by approximately a factor of 2 (Cherry, 1995), apparently due to the strain concentrations from local nonuniformities of the corroded surface.

As an example, consider a situation in which uniform corrosion penetrates to depth, $x(t)$, in a plate of thickness, W. Assuming that strength rather than ductility governs, when $x(t)$ reaches the thickness $W$, the 
plate loses all capacity to carry load. The impact of this degradation depends on the nature of the behavioral limit state. If the plate is in a state of simple tension,

$$
R(t)=F_{y} W(1-x(t) / W)
$$

and sample function $g(t)$ is simply $1-x(t) / W$. On the other hand, if the plate is stressed in flexure, the bending strength per unit width is,

$$
R(t)=F_{y} W^{2}(1-x(t) / W)^{2} / 6
$$

and $g(t)=(1-x(t) / W)^{2}$. Thus, small errors in estimating $x(t)$ would have a more significant impact on flexural strength than on tensile strength. Moreover, as damage progresses and $x(t)$ increases, the governing limit state may change during the service life of the component, creating an unanticipated and potentially dangerous situation. Since $x(t)$ actually is modeled as a random process, $X(t)$ (eg., penetration of corrosion, modeled in Section 2), the probability distribution of $X(t)$ plays a key role in the condition assessment.

\subsubsection{Fragility modeling of steel containments and liners}

Probabilistic models of resistance of steel containments and liners are integrated with stochastic load models to develop fully-coupled time-dependent reliability assessement tools. An intermediate step in the development of fully-coupled reliability analysis procedures is the fragility modeling of the containment. Fragility analysis is a relatively simple but powerful technique for assessing the capability of a structural system to withstand specified (sometimes referred to as screening or review-level) events in excess of the design-basis event. This process sometimes is referred to as a "safety margin analysis." During the last decade, it has been used to determine the capability of NPP structural components and systems to withstand, with high confidence, review-level earthquakes of a prescribed level in excess of the safe-shutdown earthquake (SSE). For example, the review earthquake might be set at $0.30 \mathrm{~g}$ if the design-basis SSE were $0.17 \mathrm{~g}$. The basic idea is that if the system can be shown to perform safely at the review level, it is judged sufficient for public safety regardless of what the actual (unknown) hazard might be.

A margin analysis has at least three advantages over a fully coupled reliability analysis:

(1) The probability distribution of the hazard or the structural action caused by it is not required (although some general idea of the potential hazard must be available in order to arrive at a sensible review level event). Design-basis events for NPP structures are very rare, and determining their probability models is difficult because of the paucity of data. Extrapolating such probability models well beyond the realm of observation creates a large source of uncertainty. In analyzing seismic hazards in the Eastern United States, for example, estimated probabilities that earthquakes in excess of the design basis occur annually can vary over two or more orders of magnitude, depending on which one of several (credible) hypotheses regarding seismic source zones is made.

(2) The convolution of hazard and resistance needed to determine probabilities of failure is avoided.

(3) The difficulty in interpreting the resulting probabilities is avoided. Because of the large uncertainties in hazard analysis, these probabilities may span three or more orders of magnitude (Ellingwood, 1990, 1992, 1994). The selection of a numerical value for assessment purposes (e.g., mean, median, mode), let alone its use as as a regulatory target, is very difficult.

A fragility analysis and margins assessment of a structure avoids these difficulties in interpretation, yet enables vulnerabilities in the structure to be identified because of the supporting system analysis that underlies the fragility model. While a margins analysis is not as informative as the measure of safety obtained from a fully 
coupled PRA, it retains many of the desirable features of the structural system reliability analysis and is easier to perform.

The fragility of a component or system, such as a steel containment-or liner, is defined as its probability of "failure," conditioned on a level of demand (from ground motion, wind velocity, internal pressure, etc.) The definition of failure depends on the performance requirements of the component or system considered. In a steel containment or liner, failure is assumed to occur when (generally inelastic) deformations are large enough to interfere with the operation of attached equipment, when pressure retention is lost or leakage above a tolerable level occurs.

The lognormal distribution is the most common way to model fragility (Kennedy and Ravindra, 1984);

$$
F_{R}(x)=\Phi\left(\frac{\ln \left(x / m_{R}\right)}{\beta_{R}}\right)
$$

in which $m_{R}=$ median capacity (expressed in units that are dimensionally consistent with the demand parameter) and $\beta_{R}=S D(\ln R)$, or standard deviation in $\ln R$, describing the inherent randomness in the

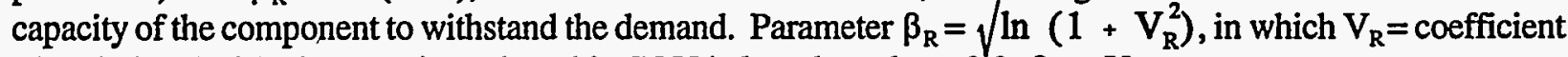
of variation (COV) in capacity; when this COV is less than about $0.3, \beta_{R}=V_{R}$.

Additional uncertainties in component capacity arise from assumptions made in the structural system analysis, limitations in the supporting statistical database, and similar factors. These modeling uncertainties can be taken into account, to first order, by assuming that the median capacity is a random variable. Accordingly, $m_{R}$ in Eqn 4.10 is replaced by random variable, $M_{R}$, assumed to be lognormal with median $m_{R}$ and logarithmic standard deviation, $\beta_{\mathrm{U}}$. The fragility thus becomes a random function of random variable, $\mathrm{M}_{\mathrm{R}}$. A family of lognormal distributions, described by the fragility parameters $\left(m_{R}, \beta_{R}, \beta_{U}\right)$, displays the overall uncertainty in the conditional component failure probability at any value of $\mathrm{x}$, as illustrated in Figure 4.2, where the graphs illustrate the 5 to 95 percentile fragility curves. The mean fragility can be shown to be (Ellingwood, 1994),

$$
\bar{F}_{R}(x)=\Phi\left(\frac{\ln \left(x / m_{R}\right)}{\sqrt{\beta_{R}^{2}+\beta_{U}^{2}}}\right)
$$

This mean fragility also is illustrated in Figure 4.2. Note that it has a different slope than the family of curves.

One uses the component fragility in a margins assessment to identify a level of demand at which there is a high confidence that the component will survive. It has been common in structural design and safety checking to use a nominal or characteristic strength that has a small probability, typically 0.05 , of not being attained. (This so-called 5 percentile value of strength, or 5 percent exclusion limit, is the basis for characteristic strengths now being recommended in the new limit state-based Eurocodes that are being implemented in Western Europe.) Figure 4.2 shows that in the presence of uncertainties arising from insufficient data, the 5 percent exclusion limit has a frequency distribution, assumed to arise from uncertainties in estimating the median, $m_{R}$. The lower $\alpha$-fractile of this frequency distribution is a number, $R_{\alpha}$; one might say that the probability of surviving an event with intensity $R_{\alpha}$ is 95 percent with confidence $(1-\alpha) 100$ percent. Conversely, one can obtain $R_{\alpha}$ as,

$$
R_{\alpha}=m_{R} \exp \left(-1.645 \beta_{R}+k_{\alpha} \beta_{U}\right)
$$


in which $k_{\alpha}=\Phi^{-1}(\alpha)$. In seismic margins analysis, it has been customary to set $\alpha=0.05$ and refer to $R_{\alpha}$ as the HCLPF, or "high-confidence, low probability of failure, value. This HCLPF can be expressed as,

$$
\mathrm{HCLPF}=\mathrm{m}_{\mathrm{R}} \exp \left[-1.645\left(\beta_{\mathrm{R}}+\beta_{\mathrm{U}}\right)\right]
$$

The HCLPF is akin to a lower tolerance limit, but in a Bayesian rather than a classical statistical sense.

Eqns 4.10 and 4.11 simply display the uncertainty in the component capacity. The fragility parameters must be determined from structural analysis, examination of available statistical data, and expert judgement. To illustrate, suppose that it is desired to perform a margins analysis of a Mark I steel containment drywell subjected to internal pressure, $P_{\mathrm{a}}$. The capacity can be analyzed as the product of a series of factors (cf Eqn 4.4 and 4.7),

$$
R=\left(I I F_{i}\right) P_{a}
$$

in which $P_{a}=$ design-basis pressure (typically $40-60$ psig for a BWR Mark 1 ) and $F_{i}=$ random factors which, collectively, represent the difference between strength of the containment in-service and the assumed design strength. Assuming that the factors $F_{i}$ are mutually statistically independent, the median and variability in $R$ are,

$$
\begin{aligned}
& m_{R}=\Pi m_{i} P_{a} \\
& \beta_{R}=\left[\Sigma \beta_{i}^{2}\right]^{1 / 2}
\end{aligned}
$$

in which $m_{i}=$ median of factor $i$ and $\beta_{i}=$ logarithmic standard deviation describing inherent randomness or uncertainty in factor $i$. As the product of independent factors, $R$ can be modeled by a lognormal distribution by virtue of the central limit theorem of probability theory.

At the current state-of-the-art, the starting point of the containment fragility analysis is the plantspecific elastic design calculations of containment strength, which usually are available. The product of factors in Eqn 4.13 can be expressed as,

$$
\text { III } F_{i}=F_{s} F_{\mu} F_{r s}
$$

Factor $F_{s}=F_{y} / F_{a l l}=P_{y} P_{a}$, in which $F_{y}=$ yield strength and $F_{\text {all }}=$ allowable stress against which design-basis pressure, $P_{a}$, was checked in design, and $P_{y}=$ pressure at which first yield occurs; $F_{\mu}=P_{u} / P_{y}$, in which $P_{u}=$ pressure at which excessive inelastic deformation of the shell occurs; and $F_{r s}=$ structural response analysis factor, describing the relative accuracy (bias) of the analysis used in the design calculations to determine containment response to internal pressure. These factors are illustrated in Figure 4.3, which shows internal pressures vs radial displacements measured in a test of a 1/6 scale model of a reinforced concrete containment with a steel liner (Walther, 1992). The behavior predicted by nonlinear finite element analyses conducted as part of a containment reliability study (Rajashekhar and Ellingwood, 1995) is shown for comparison.

The determination of the median, inherent randomness and modeling uncertainty for each factor in Eqn 4.13 requires an audit and supplemental analyses of the design calculations of a specific containment. Such a review is nontrivial and is outside the scope of the present report. For illustrative purposes, however, we might consider the following.

Structural analyses in support of containment design usually are elastic, and the design is based on allowable stress concepts: 
in which $\mathrm{F}_{\mathrm{cr}}=$ limiting stress (yield, buckling) and FS is a factor of safety, typically about 1.6. The median of $\mathrm{F}_{\mathrm{s}}$ thus would be approximately 1.7 ; the COV would equal the COV in yield strength, or about $0.07-0.11$, depending on the type of steel considered.

The containment capacity is not reached when first yield occurs. Rather, stresses can redistribute due to the ductility of the steel, while deformations increase in the radial and meridional directions. Eventually a point is reached where radial (hoop) deformations reach an unacceptable level or the tensile strength at stress raisers is approached. The pressure at which this behavior occurs is well beyond the design basis, typically at 1.5 - 2.0 times the internal pressure at initial yield. A more precise value must be determined through a nonlinear finite element analysis of the specific containment. The median of $\mathrm{F}_{\mu}$ typically would be on the order of 1.8 , with modeling uncertainty of 0.15 .

Structural response calculations nowadays are made almost exclusively using finite element analysis. Modern finite element codes are highly sophisticated, and are capable of analyzing nonlinear static and dynamic effects very accurately. For example, the ABAQUS finite element code was used recently (Casciati and Columbi, 1993) to conduct numerical experiments and to propagate uncertainties through the model to construct a J-integral fragility (CDF) for an internally pressurized pipeline with a surface crack oriented in the axial direction. Structural modeling assumptions in design tend to be conservative, however. A typical median value of $F_{r s}$ might be 1.10 , with modeling uncertainty $\beta_{U}=0.05$.

Collecting this information, one would have,

$$
\begin{aligned}
& \mathrm{m}_{\mathrm{R}}=1.7 \times 1.8 \times 1.1 \mathrm{P}_{\mathrm{a}}=3.37 \mathrm{P}_{\mathrm{a}} \\
& \beta_{\mathrm{R}}=0.11 \\
& \beta_{\mathrm{U}}=\sqrt{\left(0.15^{2}+0.05^{2}\right)}=0.16
\end{aligned}
$$

For a design-basis pressure of $40 \mathrm{psig}(276 \mathrm{kPa})$, the median containment capacity would be $135 \mathrm{psig}$ (931 $\mathrm{kPa}$ ). Independent nonlinear analyses of containments performed in other NRC-sponsored research have suggested ultimate capacities of this order of magnitude. The HCLPF capacity, from Eqn $4.12 \mathrm{~b}$, would be 86 psig ( $595 \mathrm{kPa}$ ), well above the design-basis pressure. The 86 psig capacity could be compared to the pressure from a review-level event to determine suitability for service.

In the presence of structural degradation, the fragility varies in time. If the containment were found to be in a degraded condition during in-service inspection, the fragility analysis would be similar, except that the median factors would need to take into account the loss of section or other damage; this would entail a finite element analysis of the containment under observed or postulated degraded conditions. Correspondingly, the modeling uncertainties, $\beta_{U}$, also would be increased. If $\beta_{U}$ increased by a factor of 2 , for example, a 40 percent change in $\mathrm{m}_{\mathrm{R}}$ to $81 \mathrm{psig}(558 \mathrm{kPa}$ ) would reduce the HCLPF to less than $40 \mathrm{psig}(274 \mathrm{kPa})$.

\subsection{Time-dependent reliability analysis of degrading structures}

In this section, a time-dependent reliability analysis is considered, fully coupled in the sense that knowledge of both stochastic loading and resistance are required. The areas of damage mechanics, stochastic characterization of the plant environment, service load history, and current strength are integrated to determine probability distributions of future structural safety margins or additional useable life associated with a minimum required structural capacity. 
A schematic representation of time-dependent reliability analysis of a deteriorating structure is presented in Figure 4.4 (Ellingwood and Mori, 1992). Sample functions of time-dependent resistance and discrete and continuous load processes are shown in Figures 4.4a and 4.4b; the scheme in Figure 4.4a was used to evaluate concrete safety-related structures in NPPs in previous work (Ellingwood and Mori, 1992; 1993; Mori and Ellingwood, 1993, 1994a, 1994b)

\subsubsection{Degradation independent of service loads}

It is assumed in this section that degradation is independent of the load history, and arises from a deterioration mechanism such as corrosion. To illustrate the reliability analysis of a degrading component in a simple way, the loads are modeled as a sequence of Poisson pulses and concurrently $R(t)$ decreases due to environmental attack, as described earlier. At any time, the margin of safety, $M(t)$, is,

$$
M(t)=R(t)-S(t)
$$

Making the customary assumptions that $\mathrm{R}$ and $\mathrm{S}$ are statistically independent random variables, the (instantaneous) limit state probability of component failure is,

$$
P_{f}(t)=P[M(t)<0]=\int_{0}^{\infty} F_{R}(x) f_{S}(x) d x
$$

in which $F_{R}(x)$ and $f_{S}(x)$ are CDF of $R$ and PDF of $S$ (Shinozuka, 1983). The $P_{f}(t)$ so determined provides a snapshot of safety at time, $t$, but does not convey information on how $P_{f}$ is evolving with $t$ as degradation occurs, nor on what information on future performance can be inferred from past performance. Such information is required in service life predictions and to schedule in-service inspection and maintenance. Reliability and hazard functions provide the additional required information.

The reliability function is defined as the probability that the structure survives during interval of time $(0, t)$. If $n$ events occur within time interval $(0, t)$, the reliability function for a structural component can be represented as:

$$
L(t)=P\left[R\left(t_{1}\right)>S_{1}, \ldots, R\left(t_{n}\right)>S_{n}\right]
$$

in which $R\left(t_{i}\right)=$ strength at time of load $S_{i}$. Taking into account the randomness in the number of loads and the times at which they occur as well as in the initial strength, the reliability function becomes (Ellingwood and Mori, 1992),

$$
L(t)=\int_{0}^{-} \exp \left(-\lambda t\left[1-t^{-1} \int_{0}^{t} F_{S}\left(g_{i} r\right) d t\right]\right) f_{R_{0}}(r) d r
$$

in which $f_{R_{0}}(r)=$ PDF of the initial strength $R_{o}$ (Table 4.2) and $g_{i}=$ fraction of initial strength remaining at time of load $S_{i}$. The probability of failure during $(0, t)$ is,

$$
F(t)=1-L(t)
$$

The hazard function is defined as the probability of failure within time interval $(t, t+d t)$, given that the component has survived up to time t. This conditional probability can be expressed as,

$$
h(t)=-d \ln L(t) / d t
$$


The reliability function and hazard function are integrally related:

$$
L(t)=\exp \left(-\int_{0}^{t} h(x) d x\right)
$$

If the structure has survived during interval $\left(0, t_{1}\right)$, it may be of interest in scheduling in-service inspections to determine the probability that it will fail before $t_{2}$. Such assessments can be performed if $h(t)$ is known. If the time-to-failure is $T_{f}$, this probability can be expressed as,

$$
P\left[T_{f}<t_{2} \mid T_{f}>t_{1}\right]=1-\exp \left(-\int_{t_{1}}^{t_{2}} h(x) d x\right)
$$

The hazard function for failures occuring purely by chance is constant. When aging occurs, $h(t)$ characteristically increases in time. Reliability assessments of nondegrading and degrading structural components can be distinguished by their hazard functions. Much of the challenge in structural reliability analysis of deteriorating structures lies in relating $h(t)$ to specific degradation mechanisms, such as corrosion. The common assumption in some time-dependent reliability studies that the failure rate increases linearly has been shown to be invalid for aging structures in nuclear plants. When degradation mechanisms are synergistic, $h(t)$ generally is unknown at the current state-of-the-art. In-service inspection and maintenance impact the hazard function, causing it to change discontinuously at the time that in-service maintenance is performed (see Figure 4.4(c)).

The reliability functions $L(t)$ and $F(t)$ are cumulative, that is, they describe the probabilities of successful (or unsuccessful) performance during service interval $(0, t)$. If $h(t)$ is very small numerically, $h(t)$ is approximately numerically equal to $P_{f}(t)$ in Eqn 4.18. It should be emphasized that $F(t)$ is not equal to $P_{f}(t)$ in Eqn 4.18; the latter is simply the instantaneous probability of failure without regard to previous (or future) structural performance. Failure to recognize the difference between these probabilities is a fundamental but common interpretive error.

The methods summarized above have been extended to structures subjected to combinations of structural load processes and to structural systems (Ellingwood and Mori, 1992). The reliability function has a similar appearance to that in Eqn 4.20 , but the outer integral on resistance increases in dimension in accordance with the number of components in the system. The system reliability may be evaluated by Monte Carlo simulation, using an adaptive importance sampling technique (Mori and Ellingwood, 1993) to enhance the efficiency of the simulation.

\subsubsection{Illustration of time-dependent reliability - corrosion}

The effect of degradation in component strength due to corrosion on component reliability is illustrated for a steel cylindrical shell. The sensitivity study herein identifies some of the more important parameters for condition assessment purposes. Each reliability analysis is carried out for a period of 60 years, the sum of the initial service period of 40 years and a tentative 20 -year period of continued service. A cylindrical steel shell of radius $55 \mathrm{ft}$. $(17 \mathrm{~m})$ and uniform wall thickness $h_{0}$ is subjected to accidental pressure whose nominal value, $\mathrm{P}_{\mathrm{a}}$ is $40 \mathrm{psig}(276 \mathrm{kPa})$. The shell is made of pressure vessel grade carbon steel $\mathrm{A516} / 70$, whose nominal yield stress $\mathrm{F}_{\mathrm{yn}}$ is $38 \mathrm{ksi}(262 \mathrm{MPa})$ and ultimate strength is $70 \mathrm{ksi}$ (483 MPa). The design basis is

$$
S_{m c} \succeq D+L+P_{a}
$$

where $S_{m c}=19.5 \mathrm{ksi}(134 \mathrm{MPa})$ for A516/70 steel, and D, L, $P_{\mathrm{a}}$ denote the stresses caused by the dead load, live load and pressure build-up respectively. 
According to elastic analysis, the maximum stress caused by the internal pressure inside the shell is $P_{a} r / h_{0}$ which gives the required shell thickness as $h_{0}=1.35$ in $(34 \mathrm{~mm})$. The shell wall is subject to general corrosion. The thickness at time $t$ is

$$
\begin{aligned}
& H(t)=h_{o} \quad ; t \leq T_{I} \\
& H(t)=h_{0}-c\left(t-T_{I}\right)^{m} ; t>T_{I}
\end{aligned}
$$

where $T_{I}$ is the initiation time prior to which corrosion loss is zero.

The limit state for tensile failure due to yielding under accidental pressure is

$$
p_{y}(t)-P(t)=0
$$

where $p_{y}(t)$ is the pressure corresponding to first yield and $P(t)$ is the magnitude of the accidental pressure at time $t$. This limit state is conservative, since after its first yield the cylindrical shell does not lose all its ability to resist further pressure build-up (cf Section 4.2.3).

Normalizing this limit state with the above design equation, we obtain,

$$
1.95 \times \frac{\mathrm{H}(\mathrm{t})}{\mathrm{h}_{0}}-\mathrm{Y}=0
$$

where $\mathrm{X}$ is the random yield stress and $\mathrm{Y}$ is the random accidental pressure magnitude, both normalized by their respective nominal values. $X$ is lognormal with mean 1.1 and COV 0.07 (Table 4.2). The arrival of the (normalized) accidental pressure $Y$ takes place according to a stationary Poisson pulse process, with an assumed mean rate of 0.0017 per year. The CDF of Y is Type I with mean 0.8 and COV 0.20 (Table 4.1). Rate parameter $C$ is assumed to be either deterministic or lognormal, with mean 0.0091 in $(231 \mu \mathrm{m})$ and COV of 0.30 , to illustrate the sensitivity of the reliability to randomness in $C$. The initiation time $T_{I}$ is assumed to be a lognormal random variable with mean of $10 \mathrm{yr}$ and COV 0.30 . Time order parameter $\mathrm{m}$ is deterministic and equal to 0.7 .

Figure 4.5(a) shows the hazard function $h(t)$ and the failure probability $F(t)=1-L(t)$ in Eqn 4.21 (probability that life $T$ of the structure is less than $t$ ) of the structure for periods of up to 60 years when $C$ is deterministic. It can be seen that if the corrosion loss is neglected, $h(t)$ remains constant, implying that the instantaneous failure probability does not increase with time. On the other hand, by taking corrosion into account, the effect of aging can be seen clearly. Figure 4.5(b) compares reliability estimates for the two cases when corrosion rate parameter, $C$, is modeled as random or deterministic. Provided that $C$, as a fraction of component thickness, or the mean rate of occurrence of the significant load are small, the results are practically the same. When $\mathrm{C}=500 \mu \mathrm{m} / \mathrm{yr}(0.0236 \mathrm{in} / \mathrm{yr})$, which is well above the rates indicated for uniform corrosion in Table 2.1, randomness in $C$ has less than an order-of-magnitude effect after 60 years. The failure probabilities in all these examples are estimated by Monte-Carlo simulation with sufficient samples to keep the standard error in the estimates below $0.5 \%$.

Figure 4.6 shows the effect of making the occurrences of $P_{a}$ less frequent. By lowering the mean rate $\lambda$ to 0.0001 per year, both $h(t)$ and $F(t)$ decrease by more than one order of magnitude from the above case. Figure 4.7 compares the failure probabilities completed with and without an induction period prior to corrosion initiation. The hazard functions in Figs. 4.5-4.7 clearly are nonlinear. The assumption of a linear failure rate may be conservative for structural components subjected to corrosion.

The above tensile limit state is a linear function of the thickness $H(t)$. By considering a flexural limit state, which is a quadratic function of $H(t)$, there is a more pronounced effect of section loss. Consider a plate of unit width made of the same material as described above, subject to the same corrosion mechanism, and 
having the same initial thickness of 1.35 in $(34.3 \mathrm{~mm})$. If the plate is subjected to live load (assume dead load is negligible), the design basis then is,

$$
0.6 \mathrm{~F}_{\mathrm{yn}} \mathrm{S}_{\mathrm{xn}} \geq \mathrm{L}
$$

where $S_{x n}$ is the nominal value of the elastic section modulus. The limit state is

$$
\mathrm{F}_{\mathrm{y}} \mathrm{S}_{\mathrm{x}}-\mathrm{L}=0
$$

Upon normalization as before, this becomes

$$
1.67 X\left(\frac{H(t)}{h_{0}}\right)^{2}-Y=0
$$

In this example, strength $\mathrm{X}$ is assumed to be a lognormal random variable with mean 1.1 and $\mathrm{COV} 0.11$. $\mathrm{Y}$ is a Type I random variable with mean 0.3 and COV 0.50 , which occurs as a stationary Poisson pulse process with mean rate of 0.5 per year.

Figure 4.8 shows the effect of general corrosion is more pronounced on the flexural limit state than on the tensile limit state. Hazard function $h(t)$ at 60 years is more than one order magnitude lower if corrosion is not taken into account. Figure 4.9 shows the level of conservation in the reliability prediction if the time to initiate corrosion is neglected.

\subsubsection{Degradation dependent on service loads}

Damage accumulation due to fatigue depends on the load history. Despite advances in fatigue and fracture analysis, the use of $\mathrm{S} / \mathrm{N}$ diagrams for constant amplitude cycling, coupled with the Palmgren-Miner rule for dealing with variable amplitude cycling, still is state-of-the-art for predicting fatigue crack initiation or service life (Banon et al, 1994a; Kung and Wirsching, 1993).

If degradation occurs due to fatigue damage accumulation under variable amplitude loading during interval $(0, t)$, we have from Eqn 2.10,

$$
D(t)=\sum_{i=1}^{N(l)} C^{-1} S_{i}^{m}
$$

in which $N(t)=$ random number of load cycles. Failure is assumed to occur when $D(t)>\Delta$, in which $\Delta=$ random variable that accounts for uncertainty in Miner's rule at failure. Parameter $\Delta$ often is assumed to be lognormal, with median $\mathrm{m}_{\Delta}=1.0$ and SD $(\ln \Delta)=0.30$ to 0.60 (Committee, 1982; Yao, et al, 1986; Torng and Wirsching, 1991). If the damage increments are small, $N(t)$ is large, and the load (stress) process is stationary and narrow band, the expected value of $D(t)$ is,

$$
\begin{aligned}
& E[D(t)]=E[N(t)] E\left[C^{-1} S^{m}\right] \\
& =(v t) C^{-1} E\left[S^{m}\right]
\end{aligned}
$$

in which $E[N(t)]=v t, v=$ mean cycling rate, and $E\left[S^{m}\right]$ is the mth moment of stress range $\Delta S$. The latter is determined from,

$$
E\left[S^{m}\right]=\int_{0}^{-} s^{m} f_{s}(s) d s
$$


in which $f_{s}(s)=P D F$ of stress range, which must be determined from the operating load history. The variance of $\mathrm{D}(\mathrm{t})$ is more difficult to obtain, but is proportional to $1 / t$. Thus, assuming that $t$ is large, the fatigue life can be defined at the point where $E[D(t)]=1.0$ (Lutes, et al, 1984). This assumption leads to the relation,

$$
\left(v \mathrm{~T}_{\mathrm{f}}\right) \mathrm{E}\left[\mathrm{S}^{\mathrm{m}}\right]=\mathrm{C}
$$

which resembles the traditional Basquin equation, in which the deterministic parameters are replaced by mathematical expectations, and $T_{f}=$ time to failure.

If the excitation $S(t)$ is modeled as a narrow-band Gaussian process with zero mean and variance $\sigma_{\mathrm{s}}{ }^{2}$, the peak amplitudes can be described by a Rayleigh distribution:

$$
F_{\mathrm{S}}(\mathrm{x})=1-\exp \left(-\mathrm{x}^{2} / 2 \sigma_{\mathrm{s}}^{2}\right) ; \mathrm{x} \geq 0
$$

Under these conditions, $\mathrm{E}\left[\mathrm{S}^{\mathrm{m}}\right]$ in Eqns 4.34 and 4.35 is,

$$
E\left[S^{m}\right]=\left(\sqrt{2} \sigma_{s}\right)^{m} \Gamma\left(\frac{m+2}{2}\right)
$$

Recent extensions of the Palmgren-Miner cumulative damage hypothesis have been made for both broad-band and narrow-band stochastic excitation (Sarkani, et al, 1994). Several power spectral density functions (PSDs) have been suggested to model Gaussian excitations in the frequency domain; sample functions, $s(t)$, in the time domain then can be determined by simulation. When the excitation is broad band, cycles are defined by the "rainflow" cycle-counting procedure. A damage correction factor for broad-band (rainflow, time domain analysis) damage accumulation was developed by simulation to enable the simpler Rayleigh closed-form approximation in Eqs 4.30 and 4.31 to be used (Wirsching and Light, 1980).

Equations $4.32-4.37$ can be used to predict time to initiate a detectable defect (say, $6 \mathrm{~mm}$ in size) or to predict overall fatigue life. However, the residual strength at an arbitrary time, $t$, in the interval $0 \leq t \leq T_{f}$ cannot be determined. Fracture mechanics can be used to determine residual strength or time to failure after initiation of a crack.

Crack growth can be predicted from Eqn 2.14, repeated here for convenience:

$$
\mathrm{da} / \mathrm{dN}=\mathrm{C}(\mathrm{Y} \Delta \mathrm{S} \sqrt{\pi \mathrm{a}})^{\mathrm{m}}
$$

in which $\mathrm{a}=$ crack size, $\Delta \mathrm{S}=$ stress amplitude, and $\mathrm{Y}=$ geometric correction factor. Assuming that the loading can be modeled as a sequence of random loads, $S_{i}$ (Casciati and Colombi, 1993),

$$
\left.\int_{a_{0}}^{a_{1}}(Y \sqrt{\Pi a})^{-m} d a\right)=C \sum_{i=1}^{N(i)} S_{i}^{m}
$$

in which $\mathrm{a}_{\mathrm{o}}=$ initial crack size, and $\mathrm{a}_{\mathrm{f}}=$ final crack size. It can be shown that if the load history is stationary and narrow-band,

$$
\lim _{t \rightarrow \infty} C \sum_{i=1}^{N(t)} S_{i}^{m}=(v t) C E\left[S^{m}\right]
$$


The similarities of the right hand side of Eqn 4.40 to Eqn 4.35 should be noted. Other, more involved equations for predicting crack growth are available. However, it is arguable whether they are any more useful in time-dependent reliability analysis than this relatively simple approach because of the numerous uncertainties in the crack growth process and the ability of any equation to model it.

Statistical models of $\mathrm{a}_{0}$ and $\mathrm{a}_{\mathrm{f}}$ must be known for Eqn 4.39 to be useful in life prediction. The initial flaw size might be assumed to be the minimum detectable flaw size. This would depend on the NDE technology, since the resolutions of the different methods are different and, what is more important, depend on field conditions. Or, $a_{0}$ might be the maximum size of flaw allowed to remain unrepaired in the structure if, following inspection, a decision is made to repair only those flaws larger than some "critical" size. The final flaw size, $a_{f}$, might be the size at the onset of unstable crack propagation, the component thickness (recommended in analyzing and ranking pipe welds for leak probabilities using the PRAISE code (Holman, 1989) or for "leak-before-break" analysis), the crack size corresponding to an unacceptable increase in component compliance, or other performance-related definition. With $a_{0}$ or $a_{f}$ defined, the probability of unacceptable defect growth can be determined from Eqn 4.39 (see, e.g. Oswald and Schueller, 1984; Ortiz and Kiremidjian, 1986).

Fatigue crack growth is a random process. In applying the Paris-Erdogan law in fatigue reliability analysis, some researchers have assumed either $m$ or $C$ to be the random variable or the random process (the other being deterministic), and some have taken them to be jointly distributed (eg, Ortiz and Kiremidjian, 1986). Rocha et al (1993) found $m$ and $\log C$ to be linearly dependent for high tensile steel. Thara and Misawa (1991) assumed C to be a non-stationary Gaussian process.

Deterministic models like Eqn. 2.14 also have been rendered stochastic by multiplying the right hand side with a non-negative random process (Lin and Yang, 1983; Spencer and Tang, 1988) as in the following:

$$
\frac{\mathrm{dA}(\mathrm{t})}{\mathrm{dt}}=\mathrm{C}(\Delta \mathrm{K})^{\mathrm{m}} \mathrm{X}(\mathrm{t})
$$

where $\mathrm{A}(\mathrm{t})$ is the random crack size and $\mathrm{X}(\mathrm{t})$ is the random process. Alternately, arguing that uncertainty in crack growth rate arises out of inhomogeneity and randomness of material properties at or near the crack-tip, others (eg, Ditlevsen, 1986; Ortiz and Kiremedjian, 1986; Dolinski 1992) have introduced a multiplicative random function of the crack-tip position, a, rather than of $t$, in their stochastic models:

$$
\frac{\mathrm{dA}}{\mathrm{dn}}=\mathrm{C}(\Delta \mathrm{K})^{\mathrm{m}} \mathrm{X}(\mathrm{a})
$$

Fatigue crack growth has often been idealized as a Markov process. In such an approach, Oswald and Schueller (1984) and Nienstedt (1990) have used probabilistic fracture mechanics to ascertain the transition probabilities. Lin and Yang (1983) adopted a diffusive Markov process to obtain the first passage time to reach the critical crack size. Ishikawa et al (1993) started with crack-growth as a general stochastic process, but subsequently approximated the process to be Markovian (continuous-time and continuous-state) assuming that the correlation function vanishes at time intervals of practical interest. Spencer and Tang (1988) modeled crack growth with a two-dimensional Markov vector process $[A(t) Z(t)]^{T}$ where $A(t)$ is the crack size as in Eqn. 4.41 and $Z(t)$ is a stationary Gaussian process. $Z(t)$ is related to $X(t)$ of Eqn. 4.41 by the transformation $Z(t)$ $=\sigma_{z} \Phi^{-1}\left(F_{x}\{X(t)\}\right]$ where $\sigma_{z}$ is the stationary standard deviation of the process $Z(t)$ and $\Phi($.$) is the standard$ normal distribution function. Spencer and Tang (1988) used a Petrov-Galerkin finite element formulation to obtain a numerical solution for the time required to reach a critical crack-size. Lin and Yang (1983) and Zhu et al (1992) used the Fokker-Planck equation to determine the pdf of crack-size as a function of time. Bognadoff and Kozin (1985) used a discrete space and discrete time uni-directional unit-jump Markov model for damage growth. Zhao (1993) has sought to improve this cumulative damage model. 


\subsubsection{Illustration of time-dependent reliability - fatigue}

To illustrate how the fatigue analysis might be performed, we consider cycling due to SRV loads. The SRV loads are unique to BWR plants. The SRVs are provided for protection against overpressure of the reactor pressure vessel during operating transients and for rapid depressurization during postulated accidents. During a SRV discharge, the suppression pool is subjected to oscillating dynamic pressures, which the containment and drywell must be able to withstand. It should be emphasized that actual SRV loads are very complex, especially when several valves participate. Accordingly, the following analysis is for illustrative purposes only.

The SRV loads are specified by the vendor. In this illustration, we will consider SRV loads from a Mark III containment that were evaluated as part of a consensus estimation survey of loads in NPPs (Hwang, et al, 1983). The total estimated number of SRV occurrences in 40 years was 1620 , implying a rate of $40.5 / y r$. The pressure fluctuations depend on the arrangement of the SRV lines; a typical frequency would be $8 \mathrm{~Hz}$. The duration of each event is approximately $0.75 \mathrm{sec}$, implying an average of 6 pressure (or stress) cycles per event. The peak design pressure is approximately $30 \mathrm{psi}(207 \mathrm{kPa})$. The A/E's surveyed believed that the design values provided by the vendor are conservative; the mean of the actual peak pressure was thought to be approximately 0.8 times the design value, or $24 \mathrm{psi}(165 \mathrm{kPa})$, and its coefficient of variation was about 0.14. Unfortunately, the stresses induced by these SRV pressures could not be determined from the information presented in the consensus estimation survey (Hwang, et al, 1983). Based on typical design capacities of containments, however, it is assumed for illustrative purposes that the cyclic stress range induced by the SRV load has a mean of less than $12 \mathrm{ksi}$ ( $83 \mathrm{MPa}$ ).

We assume a median S-N curve consistent with a Fatigue Category B detail:

$\mathrm{N} \mathrm{S}^{3.1}=3.532 \times 10^{10}$

with a coefficient of variation of 0.60 in $\mathrm{N}$ for given $\mathrm{S}$. Assuming that the damage is a lognormal random variable, the median damage accumulation to occur in periods of 30 to 60 years (from Eqn. 4.33b) and the probability of fatigue failure, are shown below:

$\begin{array}{lll}\text { Time } & \text { Median damage } & \text { Probability of fatigue failure } \\ 30 & 4.57 \times 10^{-4} & 0 \\ 40 & 6.10 \times 10^{-4} & 0 \\ 50 & 7.62 \times 10^{-4} & 0 \\ 60 & 9.15 \times 10^{-4} & \Phi(-10) \propto 10^{-24}\end{array}$

It is obvious that the probability of fatigue failure is negligible. Taking the randomness of the stress range into account has a negligible impact on this probability. Similar analysis of other details indicate that the fatigue limit state generally would not play a significant role in condition assessment of steel containments and liners.

\subsubsection{Reliability of Structural Systems}

The analysis of reliability of a system of structural components or a complex component in which several failure modes are possible is conceptually similar to that of a single component, albeit more complex mathematically. The component or system fragility or limit state probability is formulated in terms of the limit state probabilities of the individual components in the system or its failure modes, depending on how the 
system is modeled mathematically (Moses, 1990; Karamchandani, et al, 1992; Rackwitz, 1985). There are two fundamental ways of modeling a system: as a series system and as a parallel system.

In a series system, failure occurs if any component fails (or if any mode occurs, depending on how the system is modeled). If, for example, a system consists of three components (or has three possible failure modes) and if all three are necessary for proper functioning of the system (or if failure can occur in any one of three modes), system failure will occur if any of the three components fails. The system failure event, $F_{\text {sys }}$, is described in terms of the component (mode) failure events, $\mathrm{F}_{\mathrm{i}}$, as,

$$
F_{s y s}=F_{1}+F_{2}+F_{3}
$$

in which " + " here denotes union of events. In a (strictly) parallel system, failure occurs only if all components (modes) fail. For the three-component system above,

$$
\mathrm{F}_{\mathrm{sys}}=\mathrm{F}_{1} * \mathrm{~F}_{2} * \mathrm{~F}_{3}
$$

in which "*" here denotes intersection of events. Most realistic engineered systems must be modeled as combinations of unions and intersections; for example,

$$
\mathrm{F}_{\mathrm{sys}}=\mathrm{F}_{1}+\left(\mathrm{F}_{2} * \mathrm{~F}_{3}\right)
$$

meaning that the system fails if either component 1 fails or if components 2 and 3 fail together.

One generally analyzes the reliability of a system for a particular hazard, such as accidental pressure or earthquake, or collection of hazards. Such hazards are described by a CDF or complementary CDF (CCDF), several of which are summarized by the models listed in Table 4.1. The failures of the individual components may be stochastically dependent. Stochastic dependence arises from several sources. First and perhaps most significant, the structural actions induced in each component from a common hazard are related by the laws of structural mechanics. To eliminate this source of dependence properly in the system reliability analysis, the failure events in Eqns $4.41-4.43$ must be analyzed as failures conditioned on a specific level of hazard. Second, materials obtained from a common set of suppliers and common techniques employed by the contractor in construction introduce dependence, although to a lesser degree. Finally, analysis assumptions made by the designer may affect several components simultaneously.

As a example, the fragility of the system in Eqn 4.43 for a specific accidental pressure, $x$, when the individual component failures are statistically independent, is expressed in terms of the component fragilities as:

$$
F_{R_{m}}(x)=F_{R_{1}}(x)+\left[1-F_{R_{1}}(x)\right] F_{R_{2}}(x) F_{R_{3}}(x)
$$

in which $F_{i}(x)$ is the fragility of component $i, i=1,2,3$, the determination of which involves the considerations discussed in Section 4.2.3. The individual (conditional) component (or mode) failure probabilities can be determined more easily than that of the system. The determination of component and system fragilities plays a key role in seismic margins analysis, as noted previously (Ellingwood, 1994). Once the (conditional) system failure probability is known, it can be convolved with the PDF of the hazard, as in Eqn 4.18, to determine the unconditional probability of system failure.

In the analysis of many complex facilities, it has been found that the COV in global system resistance is on the order of component resistance COV, and thus is quite small in comparison with the COV in load (e.g., Banon, 1994a). Beneficial effects of redundancy often are offset by higher load variabilities. Failure margins thus are highly correlated, and thus the failure mode and capacity found from a deterministic load- 
deformation analysis extended into the inelastic range often are adequate to establish the mean (or median) for probabilistic analysis as well.

\subsubsection{Appraisal of Structural Reliability Methods}

In theory, component or system reliabilities can be computed from first-order reliability methods (FORM) or full-distribution methods (Shinozuka, 1983; Bjerager, 1990). In practice, this may not be as easy as it sounds. The determination of $F_{i}(x)$ requires statistical data on material strengths, dimensions and other basic random variables, modeling errors, and a verifiable structural model of behavior based on principles of mechanics to identify the limit condition (excessive inelastic deformation, instability, etc). There also is the numerical problem in evaluating the probability integral for realistic systems.

In classical reliability analysis, there is a presumption that the limit state function (viz Eqn 4.17) is available in closed form, and therefore that the domain of integration of Eqn 4.18 is well-defined. However, modern structural analysis often is performed using finite element methods. In contrast to classical mechanics, finite element analysis (FEA) is algorithmic in nature, yielding structural responses (forces, displacements) at discrete points, but not a general closed-form expression for the limit state function. One can, however, use FEA to develop a "response surface" approximation to the limit state surface that is sufficiently accurate to be used in reliability analysis (Bucher and Borgund, 1990; Rajashekhar and Ellingwood, 1993). One first performs the FEA at a set of carefully selected experimental points in the domain of random variables. Next, a relatively simple function or response surface - commonly a second-order polynomial - is fitted to the FEA results by regression or interpolation analysis. Once an adequate representation of the actual limit state has been achieved (and this may involve some iteration in order to minimize statistical error), the reliability analysis can be performed using the response surface.

The computation of the probability integrals in Eqn 4.18 is numerically difficult when more than a few random variables are involved. Monte Carlo methods can be used to perform these computations in approximate form (Rubenstein, 1981). The Monte Carlo approach has a number of practical advantages, particularly in a structural system reliability analysis (e.g., Moses, 1990; Torng and Wirsching, 1991): complex structural behavior can be accommodated; stochastic dependency can be modeled; the possible introduction of new random variables due to inspection and repair can be dealt with; and several failure modes can be included, e.g., fracture from overload as opposed to fracture from crack growth. Perhaps one of the most useful features of Monte Carlo simulation is the way in which it facilites visualization of the damage evolution process.

The main disadvantage of Monte Carlo methods is their lack of numerical efficiency in structural reliability analysis which involves small probabilities. If the event probabilities of interest are on the order of $10^{-\mathrm{N}}$, an unmodified random sampling procedure requires approximately $10^{\mathrm{N}+1}$ samples for the failure probability estimate to be stable. The number of samples required to achieve a given sampling error, expressed by the standard deviation, $\mathrm{SD}\left(\mathrm{P}_{\mathrm{f}}\right)$, can be reduced by modifying the random sampling process. In structural reliability analysis "importance sampling" often has been used for this purpose (Schueller and Stix, 1987; Melchers, 1990; Mori and Ellingwood, 1993). There are about 40 references on importance sampling techniques (Engelund and Rackwitz, 1993). They can be categorized by: direct, updating, adaptive schemes, or spherical schemes/directional sampling. The efficiency of these approaches depends on the number of times the structural limit state must be calculated if the $\operatorname{SD}\left(P_{f}\right)<\epsilon$; the efficiency thus is related more to the structural analysis than to the uncertainty analysis. In all methods except adaptive sampling, the positioning of the importance sampling PDF must have been achieved with a suitable algorithm, often FORM. None of the methods is optimal under all circumstances, and some experimentation is required to determine the best approach for the particular problem at hand. 


\subsection{Summary}

Structural reliability analysis provides the framework for analyzing uncertainties in structural loads, operational demands on a structural component or system, and structural capacity to withstand demands. This information can be utilized on several levels: to identify a review level event to be used in a subsequent structural analysis; to identify a high-confidence, low probability of failure capacity; to determine an instantaneous probability of component or system failure; and to determine the probability of failure during any service period.

A well-formulated system reliability analysis also can be used to evaluate fitness for continued service and to establish priorities for in-service inspection and maintenance. Existing internal events probabilistic risk assessments (PRAs) of existing plants have been used to establish priorities for inspection of the RPV and piping (Doctor, et al, 1993). Inspection priorities were established by ranking importance measures based on the contribution of component failures to core damage probability. While the information necessary to perform such an importance analysis is a product of the structural reliability analysis, such an evaluation has not yet been attempted for steel containments and liners. In the next section, prospects for using reliability methods in scheduling in-service inspection and maintenance will be considered. 
Table 4.1 Summary of structural loads on NPPs

\begin{tabular}{llllll}
\hline Load & $\lambda\left(\mathrm{yr}^{-1}\right)$ & $\tau$ & mean & COV & CDF \\
\hline \hline Dead, $\mathrm{D}$ & - & - & $1.0 \mathrm{Dn}$ & 0.07 & Normal \\
Live, $\mathrm{L}$ & 0.5 & $0.25 \mathrm{yr}$ & $0.3 \mathrm{~L}_{\mathrm{n}}$ & 0.50 & Type I \\
& & & & & \\
Pipe, $\mathrm{R}_{\mathrm{o}}$ & - & - & $0.85 \mathrm{R}_{\mathrm{o}}$ & 0.30 & Normal \\
Temp, $\mathrm{T}_{\mathrm{o}}$ & - & - & $0.85 \mathrm{~T}_{\mathrm{o}}$ & 0.16 & Normal \\
SRV discharge & & $1 \mathrm{sec}$ & $0.8 \mathrm{P}_{\mathrm{SRV}}$ & 0.14 & Normal \\
Acc. temp, $\mathrm{T}_{\mathrm{a}}$ & $10^{-4}$ & $20 \mathrm{~min}$ & $0.9 \mathrm{~T}_{\mathrm{a}}$ & 0.10 & Type I \\
Acc. press., $\mathrm{P}_{\mathrm{a}}$ & $1.7 \times 10^{-3}$ & $20 \mathrm{~min}$ & $0.8 \mathrm{P}_{\mathrm{a}}$ & 0.20 & Type I \\
Earthquake, $\mathrm{E}$ & 0.05 & $30 \mathrm{sec}$ & $0.08 \mathrm{E}_{\mathrm{s}}$ & 0.90 & Type II \\
\hline
\end{tabular}


Table 4.2 Initial resistance of steel shapes and plates

\begin{tabular}{|c|c|c|c|c|c|}
\hline Element & Steel & Prop. & nom. (ksi) & mean $(\mathrm{ksi})$ & COV \\
\hline Flanges, rolled shapes & Carbon & $F_{y}$ & - & $1.05 \mathrm{~F}_{\mathrm{yn}}$ & 0.10 \\
\hline Webs, rolled shapes & $"$ & $\mathrm{~F}_{\mathrm{y}}$ & - & $1.10 \mathrm{~F}_{\mathrm{yn}}$ & 0.11 \\
\hline Plates, flanges & $"$ & $F_{u}$ & - & $1.10 \mathrm{~F}_{\mathrm{un}}$ & 0.11 \\
\hline Plates & $"$ & $\tau_{y}$ & - & $0.64 F_{y n}$ & 0.10 \\
\hline Tension coupon & $"$ & $\mathrm{E}$ & - & 29,000 & 0.06 \\
\hline Tension coupon & $"$ & $v$ & - & 0.3 & 0.03 \\
\hline 1/4-in Plate & SA516/60 & $F_{y}$ & 32 & 48.5 & 0.07 \\
\hline 1/2-in Plate & SA516/60 & $F_{y}$ & 32 & 42.1 & 0.06 \\
\hline $7 / 16-13 / 8$ in plate & A516/60 & $\begin{array}{l}F_{y} \\
F_{u}\end{array}$ & $\begin{array}{l}32 \\
60\end{array}$ & $\begin{array}{l}47.2 \\
66.2\end{array}$ & $\begin{array}{l}0.05 \\
0.03\end{array}$ \\
\hline $11 / 4-13 / 4$ in plate & A516/70 & $\begin{array}{l}F_{y} \\
F_{u}\end{array}$ & $\begin{array}{l}36 \\
70\end{array}$ & $\begin{array}{l}48.3 \\
73.9\end{array}$ & $\begin{array}{l}0.07 \\
0.03\end{array}$ \\
\hline $1 / 4$-in liner plate & A285 & $\begin{array}{l}F_{y} \\
F_{u}\end{array}$ & $\begin{array}{l}24 \\
45\end{array}$ & $\begin{array}{l}37 \\
48\end{array}$ & $\begin{array}{l}0.04 \\
0.02\end{array}$ \\
\hline
\end{tabular}

$1 \mathrm{ksi}=6.9 \mathrm{MPa}$ 

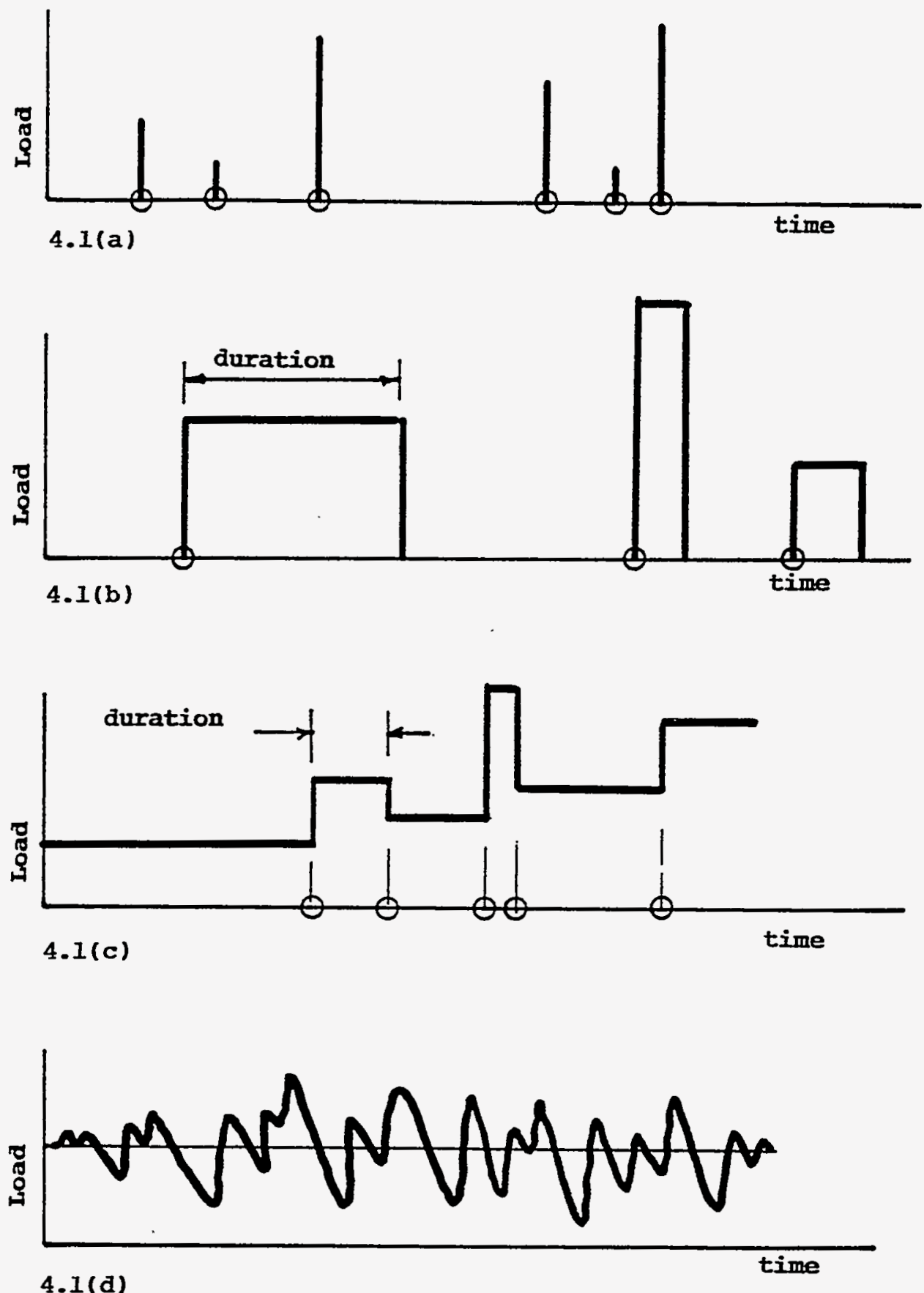

Figure 4.1 - Structural load stochastic models 


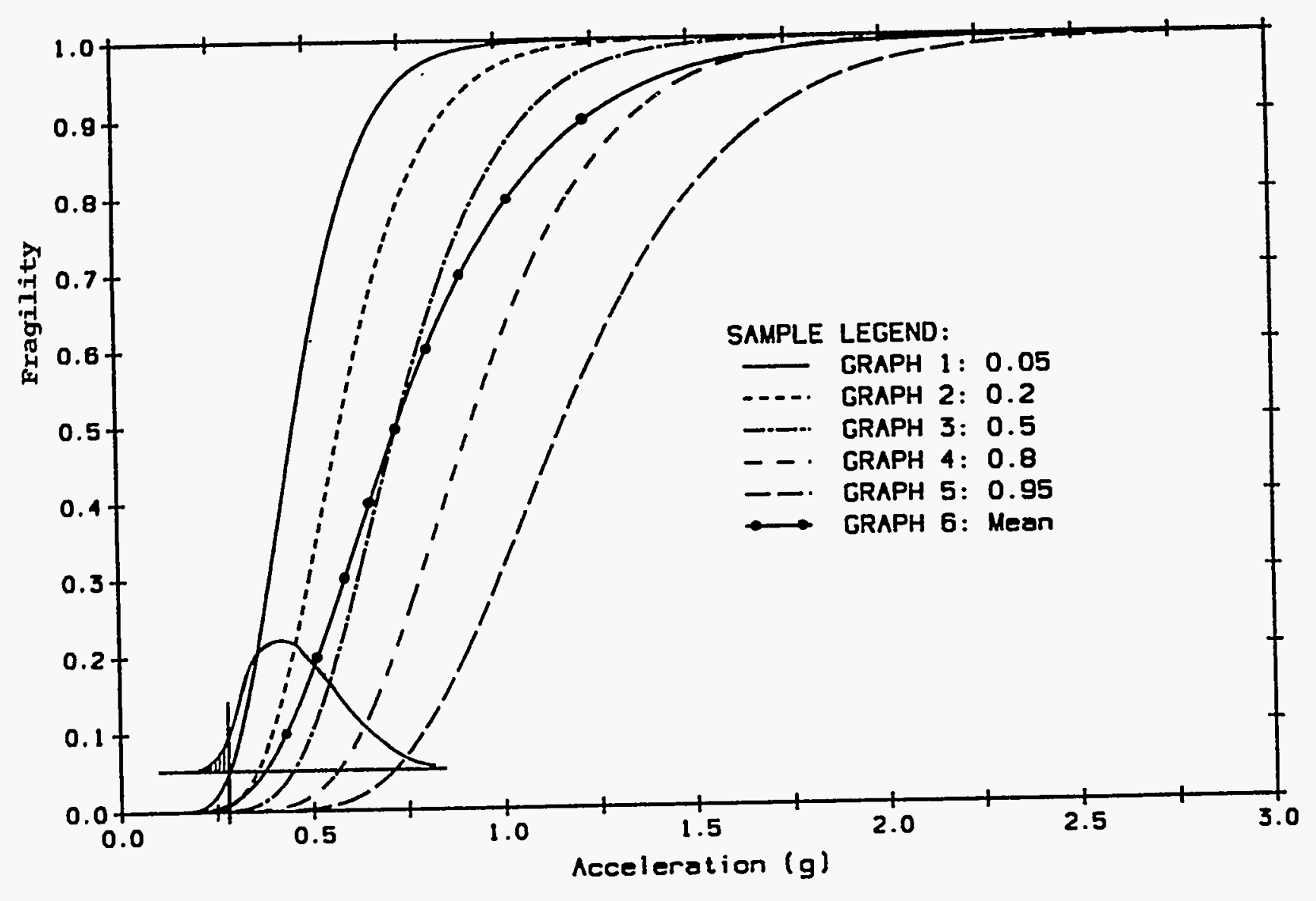

Figure 4.2 - Fragility family 


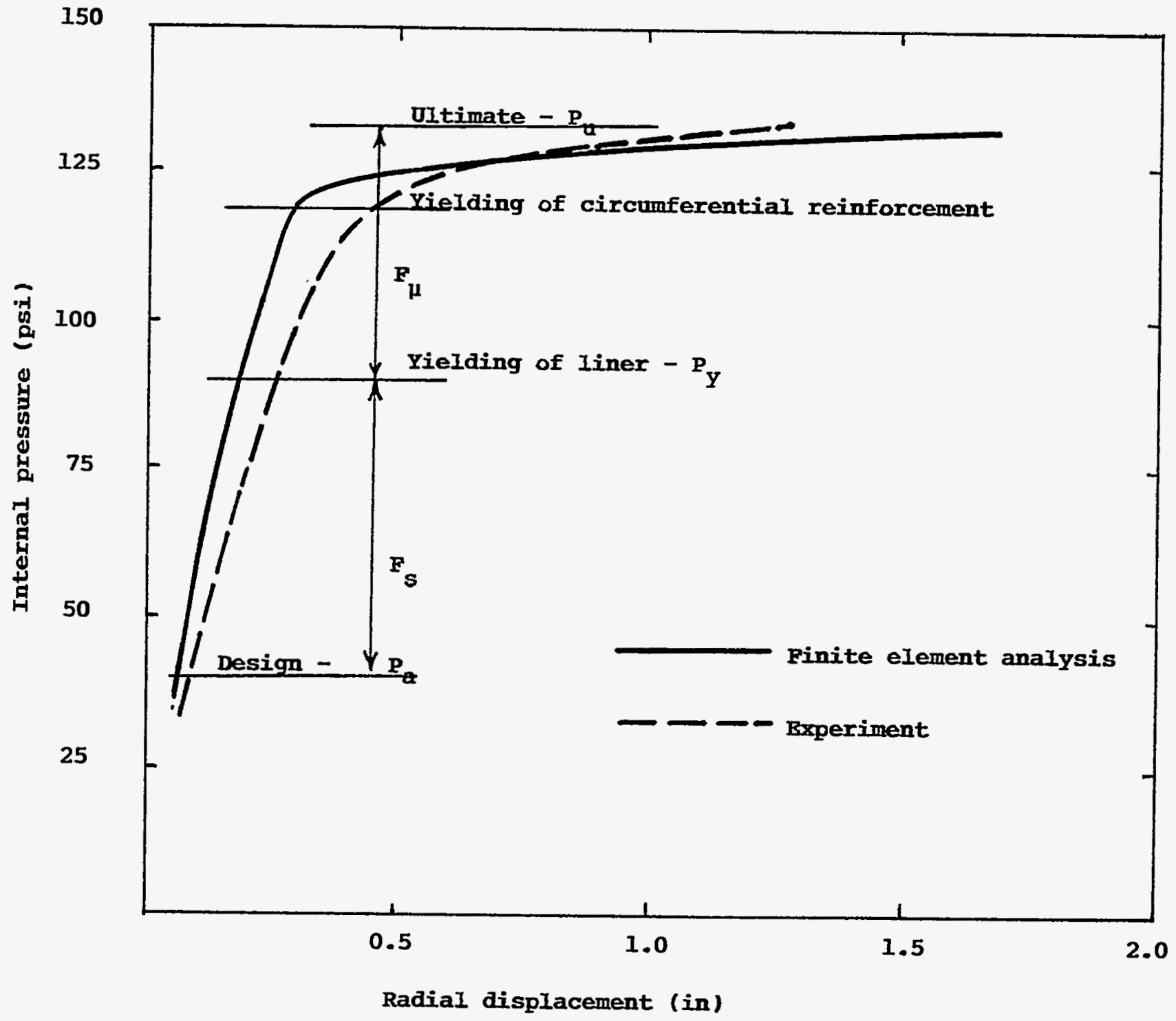

Figure 4.3 - Factors in fragility analysis 


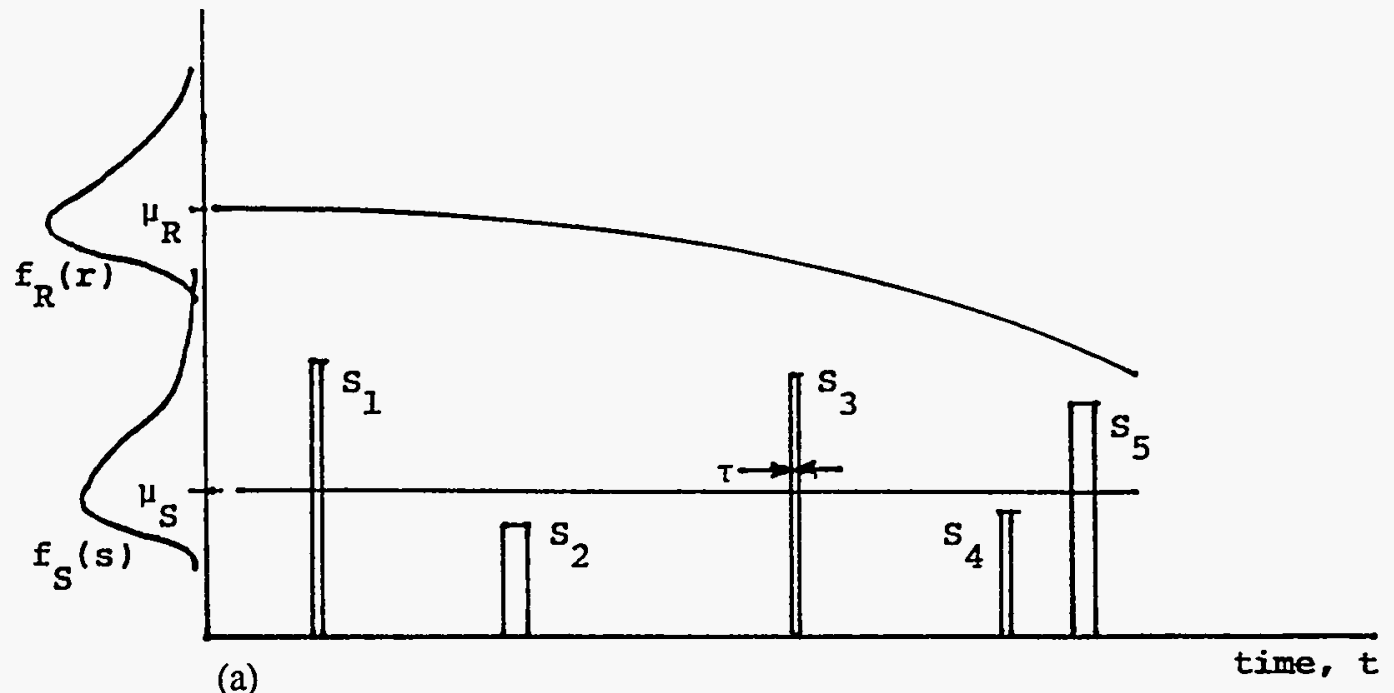

(a)

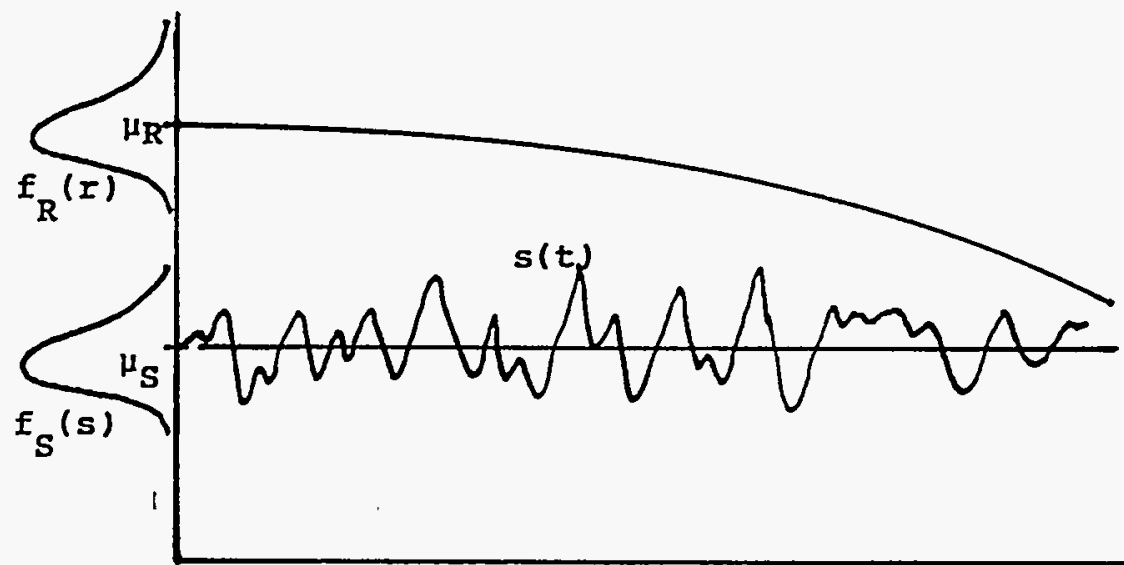

(b)

time, $t$

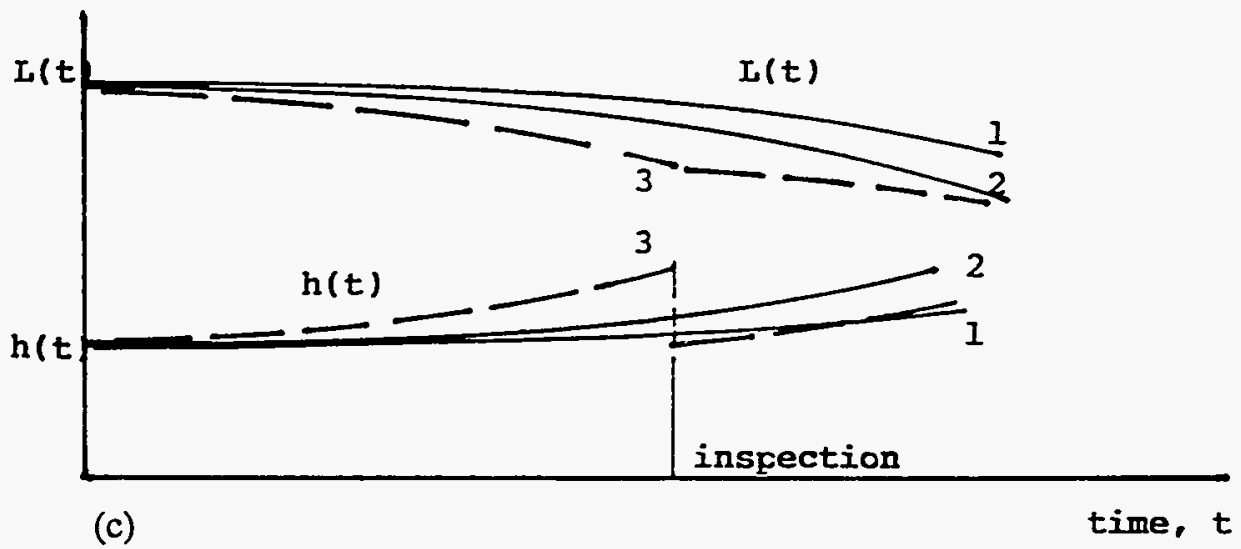

Figure 4.4 - Sample functions representing structural loads and degrading resistance 


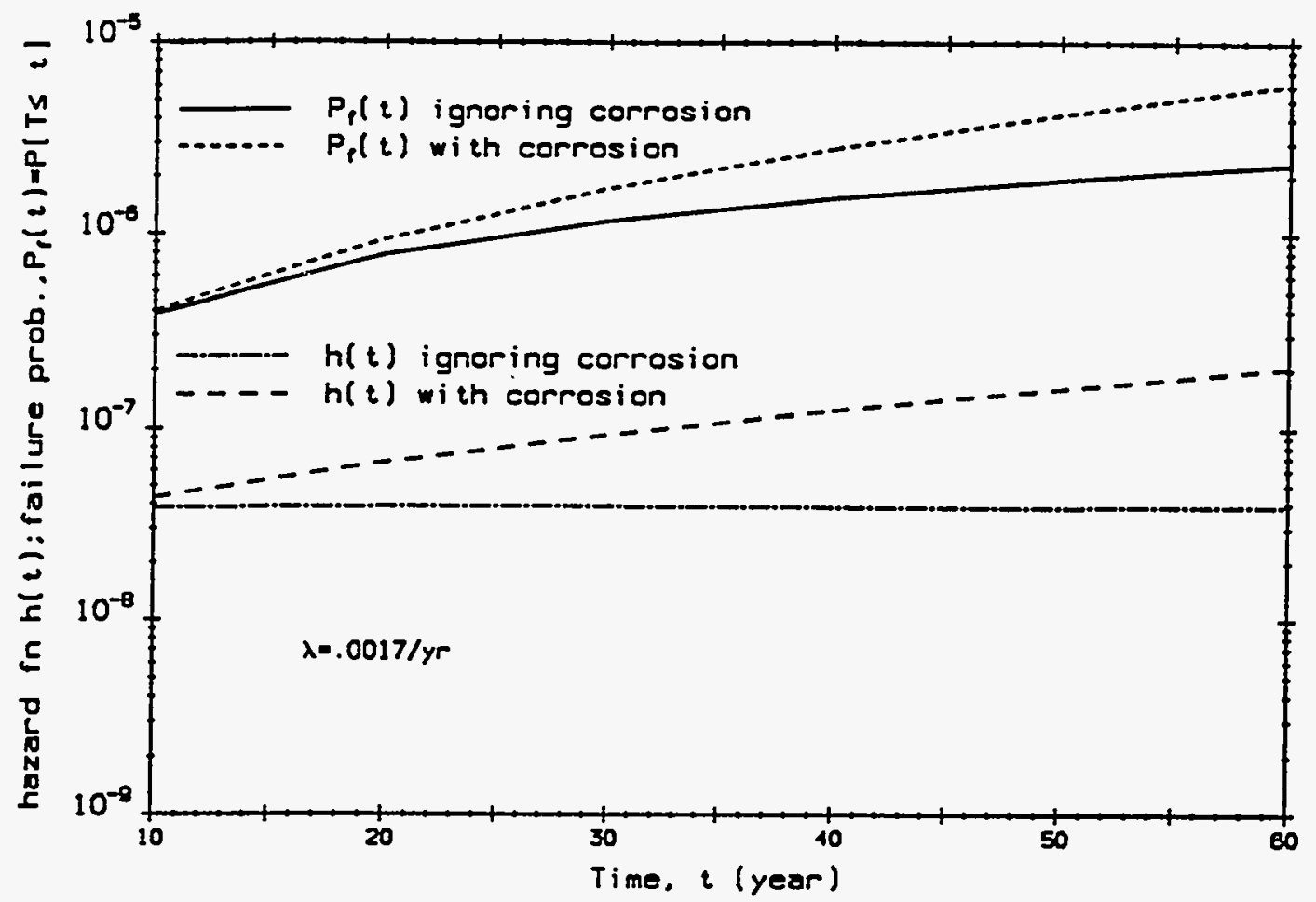

(a) Deterministic corrosion growth

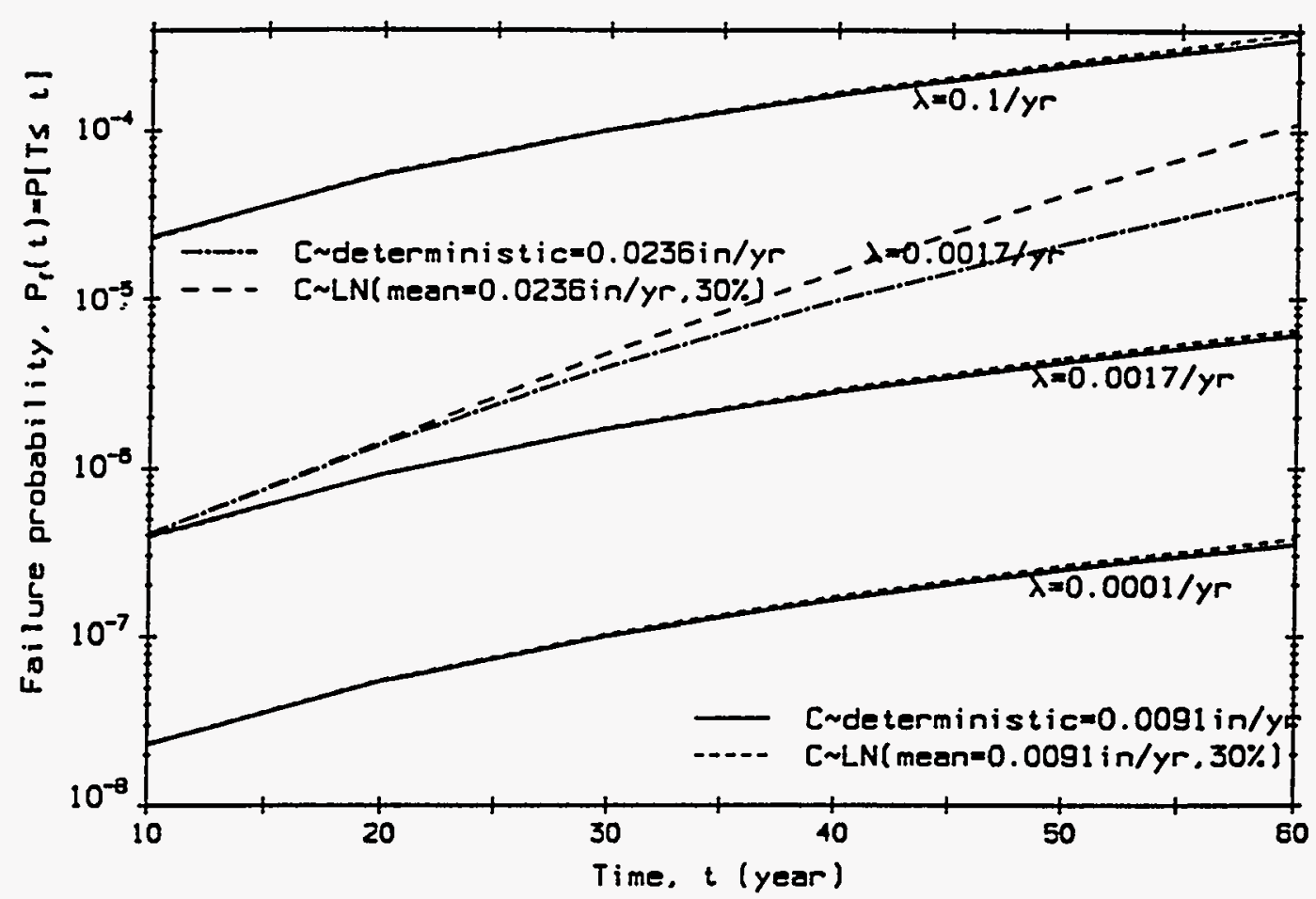

(b) Random corrosion growth

Figure 4.5 - Time-dependent reliability in tension $\left(D+P_{a}\right): \lambda=0.0017 / y r$ 


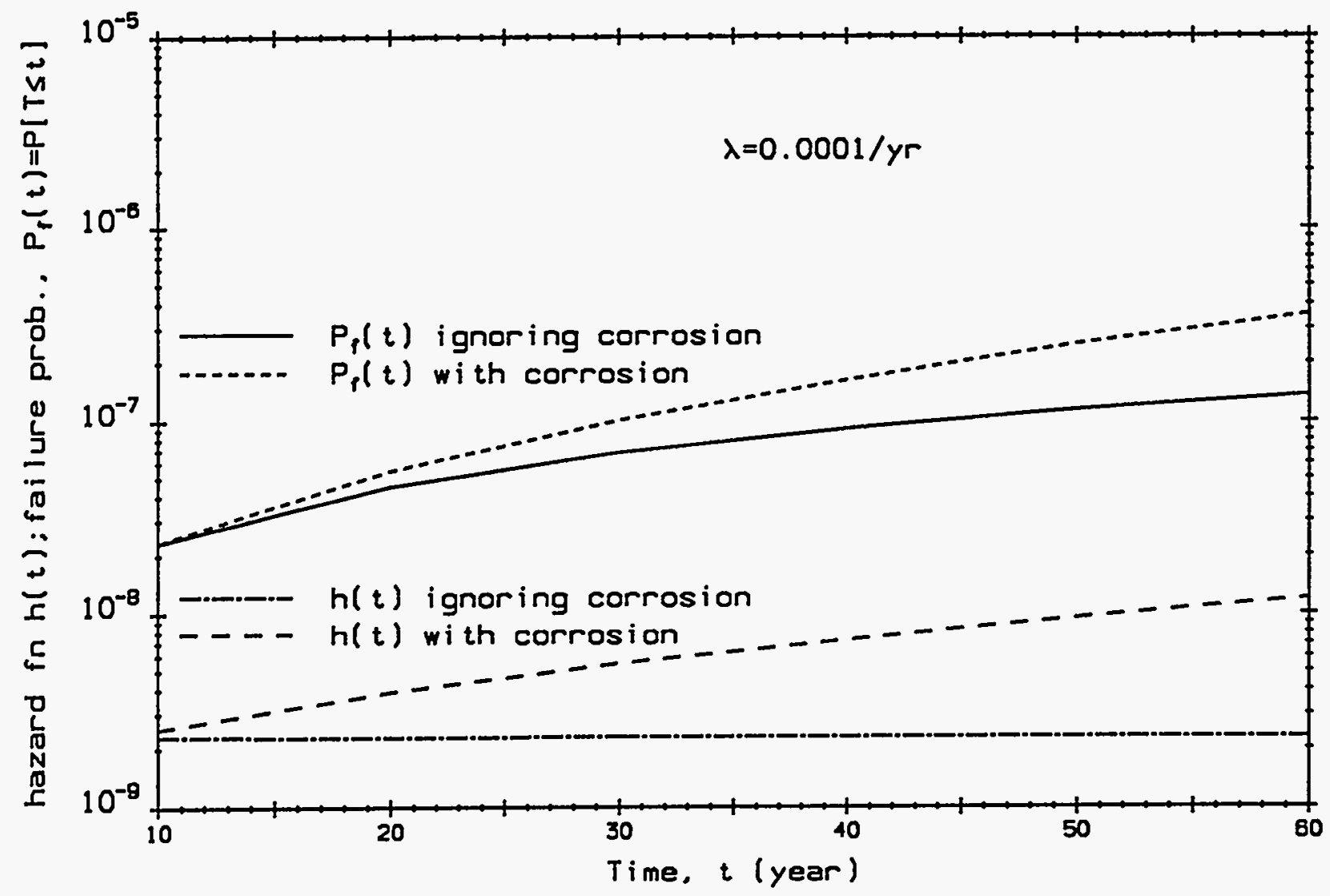

Figure 4.6 - Time-dependent reliability in tension $\left(D+P_{a}\right): \lambda=0.001 / y r$ 


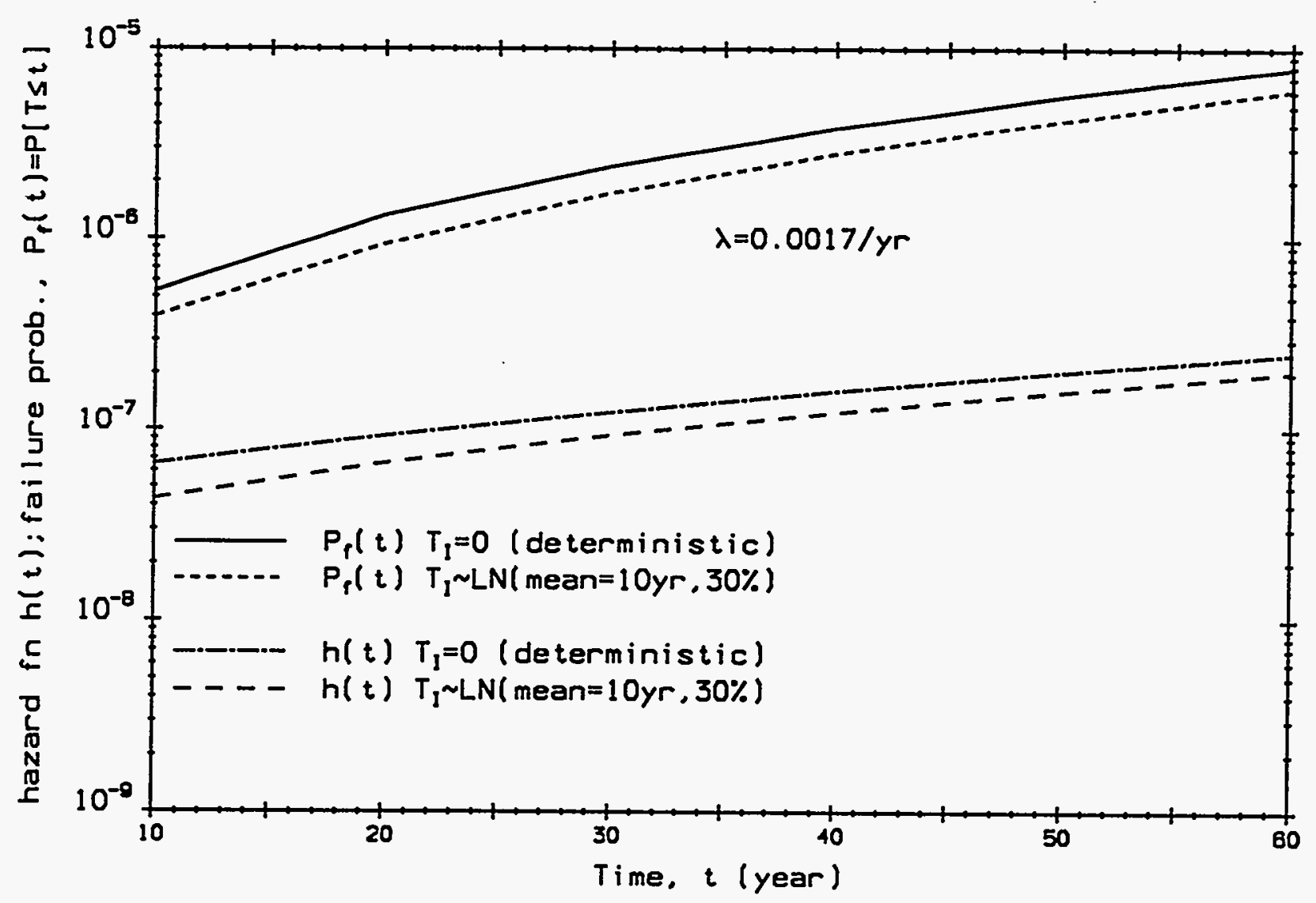

Figure 4.7 - Time-dependent reliability, with and without induction period for corrosion 


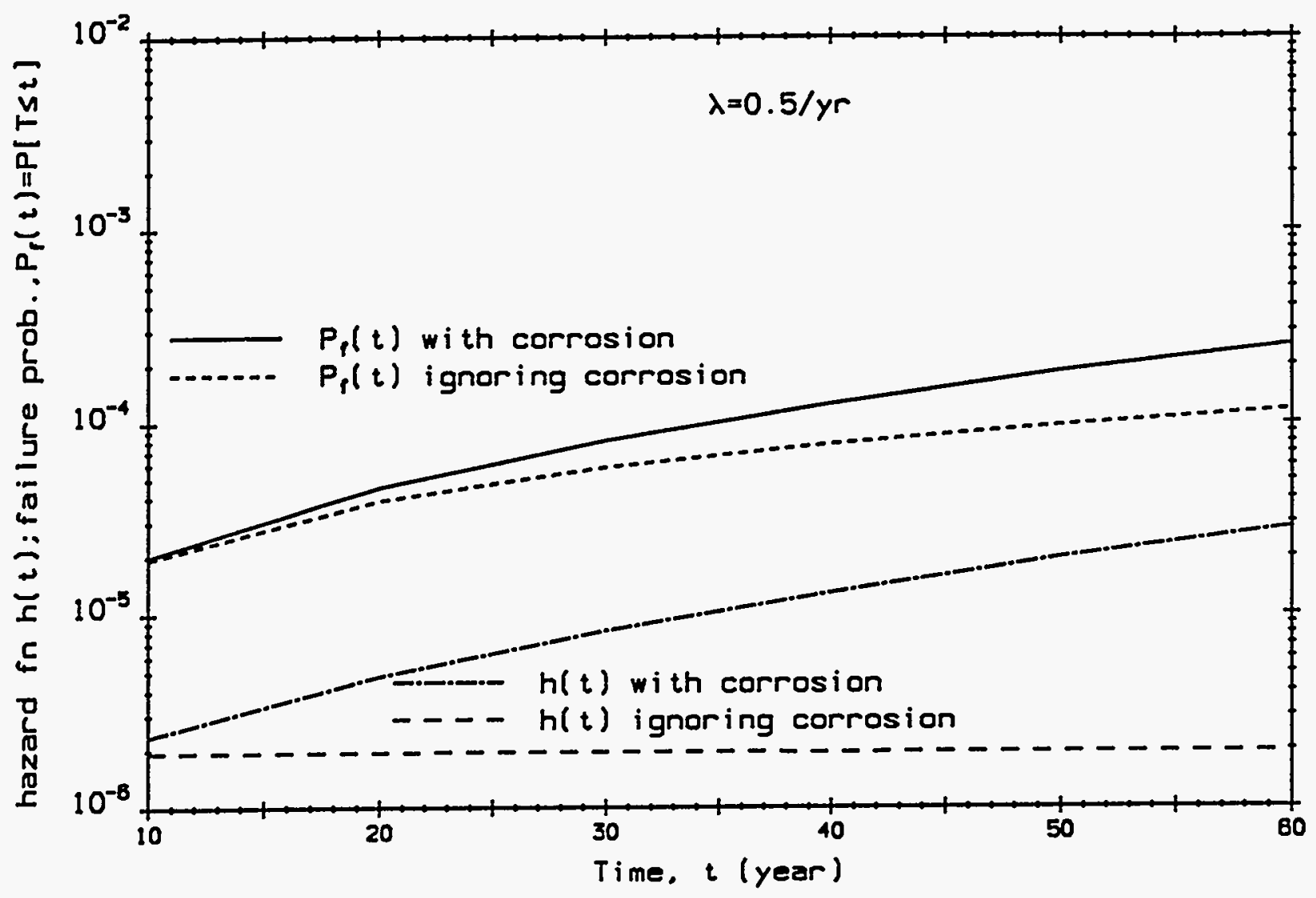

Figure 4.8 - Time-dependent reliability in flexure $(D+L)$ 


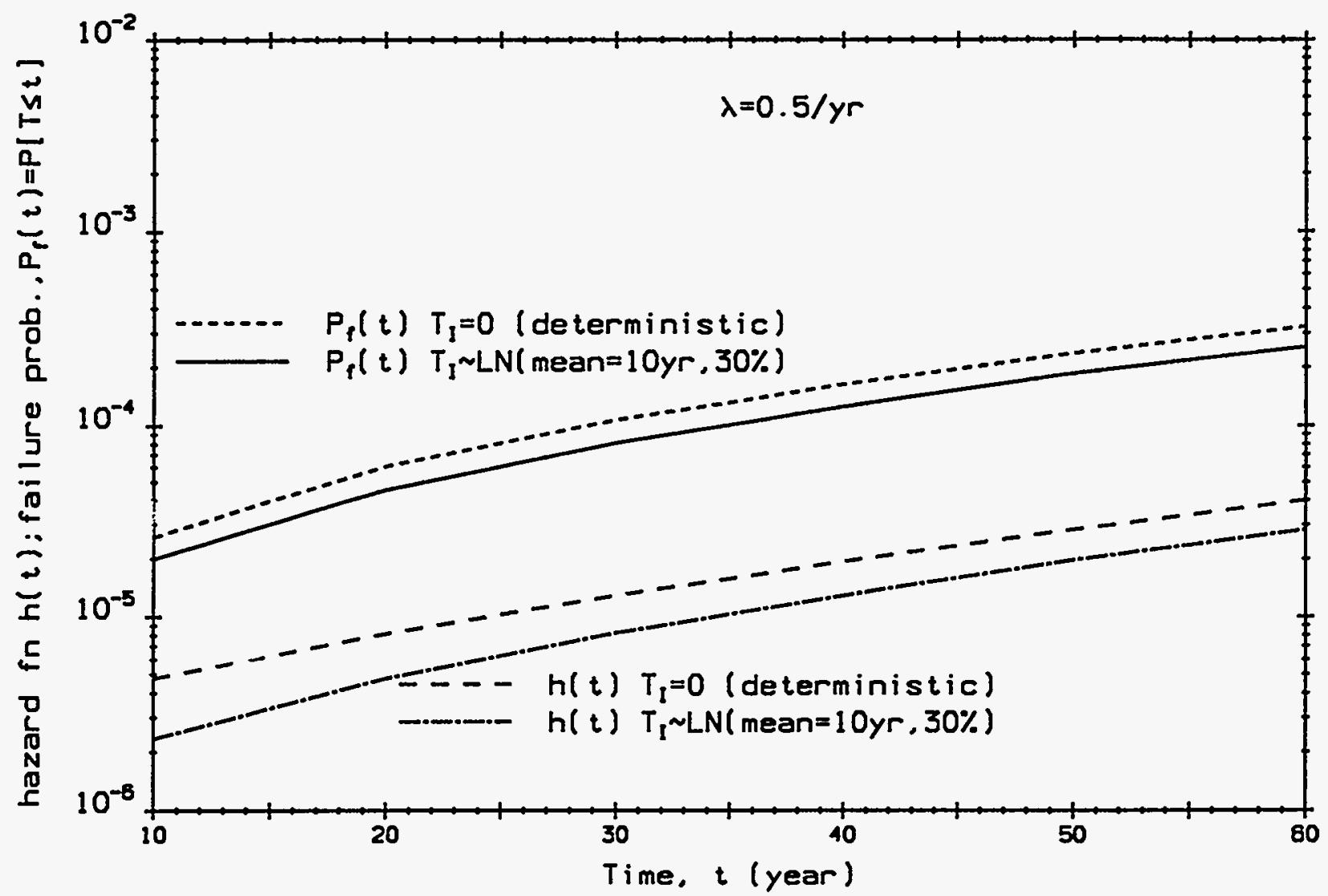

Figure 4.9 - Time-dependent reliability in flexure, with and without induction period for corrosion NUREG/CR-5442 


\section{TECHNIQUES FOR IN-SERVICE RISK MANAGEMENT}

In-service inspection provides a means for minimizing the impact of aging on structural performance. Efficient and accurate nondestructive evaluation (NDE) methods, particularly those that are noninvasive and do not disrupt the use of the structure, are essential for a properly designed condition assessment program to support facility risk management. Inspection and condition assessment identifies the cause and extent of damage, evaluates residual strength and serviceability, and provides recommendations on remedial measures (Chung, et al, 1993). Such remedial measures might include maintenance - actions that prevent or delay damage or deterioration; repair - restoration of damaged structure for continued service; or rehabilitation major modification of the structure to bring it up to current performance requirements (Wunderlich, 1991).

ASME Code Section XI Division 1, Subsection IWE currently sets inspection priorities for metal containments and liners of concrete containments. Insofar as can be determined, the ASME requirements are not based on risk analysis, and a task force is considering revised risk-based guidelines (ASME, 1992). The focus of the ASME requirements is the RPV and various piping systems. NRC inspection guidelines call for piping weld inspections based on material, heat treatment and service condition: e.g., $25 \%$ every 10 yrs; $50 \%$ every $10 \mathrm{yrs} ; 100 \%$ within two refueling cycles, etc (Holman, 1989). Most NPP facilities at present do not schedule general inspections of passive structural components regularly. Those that do inspect, do so at irregular intervals and rely almost exclusively on visual inspection. Condition assessments tend to be reactive, occurring only after there is some visible indication of damage or performance has been impaired. Operating budgets frequently do not provide for routine inspection. This attitude toward in-service inspection and maintenance must change if life-cycle risk analysis is to evolve as a strategy for facility management.

Identification of deficiencies and detection of flaws in steel structures is difficult and demanding. Although large defects usually can be found visually if the structure is accessible, sophisticated methods may be required to detect cracks or hidden defects. Incorporating this information into a reliability based structural condition assessment can have significant long-term economic and safety benefits.

\subsection{Overview of in-service inspection approaches}

A detailed condition survey is necessary at the initial stages of a inspection/maintenance/repair operation. Design documentation and records of construction and repairs, service history and environmental exposure should be reviewed at this time. The initial inspection, often performed visually, can document information on cracking, spalling, leakage, evidence of chemical attack, and other factors that may lead to structural deterioration, and indicates where to concentrate further quantitative testing procedures. A fundamental understanding of the structural performance requirements of the facility is required before initiating any in-service inspection program. The inspection plan should consider component importance; structural redundancy; accessibility; repetitive use or correlations in behavior; severity of exposure; and prior history of performance (Banon et al, 1994a; Connolly, 1995).

In-service structural inspections should be oriented toward quantifying defects in a way that can be incorporated in calculations of the degraded strength of a structural component or system. Identifying the presence of a defect without determining its size is not useful without further supplementary and quantitative evaluation. Nondestructive evaluation methods, summarized in Section 3, should be used, if at all possible. To be useful in structural condition assessment, correlations must be established between the NDE parameter measured (indentation diameter, pulse velocity, etc.) and the structural property of interest (tensile or compression strength, crack size, etc.). These correlations customarily are established by regression analysis and there often is significant scatter in the data with respect to the regression relationship. In-situ structural strength varies over a structure or even within a large structural component, depending on the environmental 
conditions to which it is exposed. Thus, repetitive sampling is necessary to obtain reliable estimates of in-situ strength. It is, of course, desirable to sample at points where the strength requirements may be severe. An appropriate sampling plan must specify the NDE procedure or procedures to be used, the zone of structure to be sampled, and the number of samples to be taken.

In cases where NDE is noninformative or inconclusive, more invasive or destructive testing may be required. Such methods have drawbacks. Destructive sampling makes post-inspection repair a critical issue and often militates against performing such tests. Small tensile coupons from steel components can be used to estimate material strength and stiffness. However, steel coupon testing is not recommended unless it is believed that the steel has been accidentally overstressed, that there is evidence of fatigue or fire damage, or that the original grade specified was not supplied. Such conditions seldom would occur in steel containments and liners in NPPs.

Load testing can be used in some cases to perform a strength evaluation of an existing structure (Hall, 1988). In a typical test, the structure is loaded in stages to a relatively high fraction (say, 75 - 90 percent) of its design strength, the load is held at each stage for a time, and deflections are measured at each stage (e.g., ACI Standard 318, 1989). The structure should show no signs of structural damage during the load test, and often a limit is placed on maximum deflection. Following unloading, the recovery of deflection is used to determine whether any permanent inelastic deformation has occurred, the occurrence of which might imply nonvisible damage. Load testing should be used only when other methods lead to inconclusive results. The tests are costly and disrupt the function of the facility unless performed during other scheduled maintenance. Moreover, recent reliability-based studies of proof load tests (Ellingwood, 1996) indicate that the test load must exceed 80 percent of the design strength before one can conclude that passage of the load test implies a measurable increase in reliability. At such load levels, there is a high probability that (repairable) damage to the structure will occur. Destructive load testing (to failure) of components is useful only if the components tested are mass-produced and easily replaced. The pressure imposed during the leakrate test performed on NPP containments is not sufficient in magnitude to verify the reliability of the containment for withstanding accidental pressures in excess of the design-basis pressure, $\mathrm{P}_{\mathrm{a}}$.

\subsection{Impact of in-service inspection on reliability}

Forecasts of time-dependent reliability enable the analyst to determine the time period beyond which the desired reliability of the structure cannot be ensured. Conversely, intervals of in-service inspection and maintenance (ISI/M)that may be required as a condition for continued operation can be determined from the time-dependent reliability analysis (Madsen, 1987; Fujita, et al, 1989; Madsen, et al, 1989).

In-service inspection reveals information about the existing structure that can be used to revise the prior estimate of strength based on the materials in the structure, construction and methods of reliability analysis described in Section 4. This change can be evaluated using Bayesian methods,

$$
f_{R}(r \mid I)=c_{i} K(I \mid r) f_{R}(r)
$$

in which $\mathrm{K}(\mathrm{I} \mid \mathrm{r})=$ likelihood function, $\mathrm{f}_{\mathrm{R}}(\mathrm{r} \mid \mathrm{I})=$ updated (posterior) PDF of structural resistance, and $\mathrm{c}_{\mathrm{i}}=$ normalizing constant. The Bayesian updating process is illustrated in Figure 5.1. The likelihood function depends on the nature of the NDE technology employed. Maintenance or repair also cause the characteristics of the strength to change by removing some of the larger defects from the structure, thereby (usually) upgrading its strength and shifting the PDF of resistance to higher strengths. This upgrading of the PDF can also be determined from Bayes theorem:

$$
f_{R}(r \mid I, M)=c_{m} K(M \mid r, I) f_{R}(r \mid I)
$$


in which $\mathrm{K}(\mathrm{Mlr}, \mathrm{D})=$ likelihood function and $\mathrm{c}_{\mathrm{m}}=$ normalizing constant. The time-dependent reliability analysis then is re-initialized following ISI/M using $f_{R}(r I I)$ or $f_{R}(r I I, M)$ in place of $f_{R}(r)$.

More generally, suppose that the margin of safety at time $t$ is $M(t)=R(t)-S(t)$, as in Eqn 4.17. The instantaneous probability of failure is $P[M(t)<0]$. Additional information gained through ISI/M about structural performance can be defined by another event, $\mathrm{H}<0$, expressed in terms of the structural variables. The revised failure probability following ISI/M is (Madsen, 1987),

$$
\mathrm{P}[\mathrm{M}<0 \mid \mathrm{H}<0]=\mathrm{P}[\mathrm{M}<0, \mathrm{H}<0] / \mathrm{P}[\mathrm{H}<0]
$$

For example, if the structure survives a load test with load magnitude $S=q$, then $H=q-R<0$, and Eqn 5.3 becomes,

$$
\begin{array}{r}
f_{R}(r \mid H)=\left[1-F_{R}(q)\right]^{-1} f_{R}(r) ; r \\
=0 ; r
\end{array}
$$

A similar approach can be taken if deflection or another structural response parameter is measured during a structural test. Moreover, if several response quantities are measured, the formulation of event $\mathrm{H}<0$ may become more complex or may be replaced by a joint event $\mathrm{H}=\left\{\mathrm{H}_{1}<0, \mathrm{H}_{2}<0, \ldots, \mathrm{H}_{\mathrm{k}}=0, \ldots\right\}$ involving both inequalities and equalities. In any event, the basic principle is the same. Subsequent reliability analysis should use $f_{R}(r \mid H)$ in place of the prior estimate, $f_{R}(r)$.

In-service inspection and repair may cause the the hazard function, $h(t)$, to change abruptly, depending on how $f_{R}(r / H)$ differs from $f_{R}(r)$ as a result of what is learned about the current condition of the structure and what repair actions are taken. A conceptual illustration of the effect of this process on the hazard function is presented in Figure 5.2. The removal of larger defects from the structure following repair reduces its conditional failure rate. As the structure ages, the failure rate increases until another repair operation occurs. The probability of structural survival during interval $\left(t_{1}, t_{2}\right)$, given that the structure has survived until $t_{1}$, is

$$
L\left(t_{1}, t_{2}\right)=\exp \left(-\int_{t_{1}}^{t_{2}} h(x) d x\right)
$$

The integrated effect of $h(t)$ in Figure 5.2 must remain below the target limit state probability.

Structures in a NPP are too complex and numerous to be completely monitored during their service life. There is a need to: (1) prioritize major components in terms of their impact on the performance of the facility; (2) identify a limited set of potential degradation sites and modes for the current operating spectrum; and (3) develop an in-service inspection plan that is directed toward (but not focussed exclusively on) those limited sites. Some sort of adaptive learning process seems most desirable for NDE. A process is envisioned that involves, first of all, inspecting a portion of structure using some noninvasive technique. If damage is found, an additional portion is inspected, perhaps with a different technique, depending on what is learned at the first inspection.

To illustrate the impact of NDE on the PDF of strength after inspection, we continue with the same example as in Section 4.3.2. Assume that rate $C$ is a lognormal random variable, with mean equal to its nominal value, and a COV of $20 \%$. The exponent $m$ remains deterministic. The randomness in corrosion loss $D(t)$ arises out of the randomness in $C$ and induction time, $T_{I}$. Since $D(t)$ is zero if $t$ is less than $t_{1}$, it has a mixed distribution with a spike (probability mass function, or PMF) at 0 . Figure 5.3 shows the distributions of $\mathrm{D}(\mathrm{t})$ at $10 \mathrm{yr}$ and $40 \mathrm{yr}$, with the mean, standard deviation and the value of the PMF at 0.

An inspection is carried out at $t=40 \mathrm{yr}$. The POD curve of the NDE instrument is 


$$
\operatorname{POD}(d)=\left\{\begin{array}{l}
0 ; d^{\prime} \leq d^{*} \\
1-\exp \left[-c\left(d-d^{\cdot}\right)\right] ; d>d^{*}
\end{array}\right.
$$

which cannot detect any section loss below $d^{*}$. The values are $d^{*}=0.06$ in $(1.5 \mathrm{~mm})$ and $c=11.5 /$ in $(0.45 / \mathrm{mm})$. A section loss of $0.15 \mathrm{in}(3.8 \mathrm{~mm})$ is observed. The error associated with this measurement is either $5 \%$ or $20 \%$. Figure 5.4 shows the updated distribution of $D(t)$. If the error of measurement can be brought down, the updated distribution becomes sharper. This is illustrated in the same figure when the measurement error is only $5 \%$.

The impact of inspection and repair on time-dependent reliability is illustrated schematically in Figure 5.5 for a reinforced concrete structure in which the reinforcement is corroding (Mori and Ellingwood, 1994a; 1994b). Two alternate NDE inspection methods are envisioned in this example. Method 1 has a capability of detecting flaws causing a strength reduction of $1 \%$, but is relatively expensive, and thus is performed infrequently. Method 2 has a capability of only detecting flaws causing a strength reduction of $8 \%$ or more, but is relatively inexpensive and so can be performed every 10 years. It is assumed that any repair following NDE fully restores the component to its original strength. Both methods are equally effective in maintaining the limit state probability of the component below approximately 0.00015 , so the selection of an appropriate method must hinge on other factors. Some ISI/M strategies may involve frequent inspection with partial repair while others involve infrequent inspection with thorough repair. Accessibility and potential hazard to the inspector are two important issues that need consideration. Any ISI/M program must represent a compromise between reliability, cost and damage detection.

\subsection{Life-cycle cost analysis}

Periodic in-service inspection followed by suitable repair may restore a degraded structure to nearoriginal condition. Since inspection and maintenance are costly, there are tradeoffs between the intervals, extent and accuracy of inspection, required reliability, and cost of facility risk management. An optimum ISI/M program might be obtained from the following constrained optimization problem:

Minimize: $C_{T}=C_{\text {ins }}+C_{\text {rep }}+C_{f} F(t)$

Subject to:

$$
\begin{aligned}
& F(t) \leq P_{f o} \\
& g_{i}()>0 ; i=1,2, \ldots M \\
& h_{j}()=0 ; j=1,2, \ldots, N
\end{aligned}
$$

in which $C_{T}=$ total cost, discounted to present worth; $C_{\text {ins }}=$ cost of inspection (a function of NDE method and frequency of inspection); $C_{\text {rep }}=$ cost of repair (dependent on labor, material and out-of-service costs); $C_{f}=$ economic and social cost of failure (including injury or mortality, loss of reputation and social disruption); $\mathrm{P}_{f_{0}}$ is the target reliability set by regulatory authority; and $g_{i}()$ and $h_{j}()$ are inequality and equality constraints on structural behavior (for example, on maximum deflection, discrete plate thicknesses, etc).

A significant database is required to determine these costs for steel containments and liners. In the absence of such data, sensitivity studies and exploration of different risk management scenarios may help guide informed policy decisions. This approach has been applied successfully to the evaluation of concrete shear wall structures in nuclear plants (Mori and Ellingwood, 1993), where it was found that the optimal policy (for 
degrading reinforced concrete structural components) was to perform ISI/M at essentially uniform intervals over the service period.

Modern risk analysis focuses on the expected failure cost, $C_{\mathrm{f}} \mathrm{F}(\mathrm{t})$, the third term in Eqn 5.7, suggesting that risk involves both probability and consequences. Low probability events can be very risky if associated with inordinate failure costs (e.g, ASME, 1992). There is more uncertainty in the likelihood of a rare event than in its consequences. While appearing to rationalize the decision process, minimum expected cost decision-making does have some shortcomings (Banon 1994b). Perhaps foremost is how to quantify intangible costs such as loss of life or environmental pollution associated with rare events. It also is difficult to compare events with high-cost and low probability of failure to alternatives involving low cost but high probability of failure. The product $C_{f} F(t)$ is the same in both cases, but the decision preference is not. The development of public policies with regard to mitigation of fatalities from automobile vs commercial aviation is a case in point.

\subsection{Measures of risk}

Risk assessment methods must be consistent for different applications in order to produce coherent policy and regulations. Proper facility risk management involves maintaining a level of safety in performance that is acceptable to society and that is understood by the people that incur the risks. A sound risk management strategy requires (Pate-Cornell, 1994) economic efficiency; equity (no individual should incur excessive risk); maintains risk at or below de minimis levels (below regulatory concern); and distinguishes between risks that people incur voluntarily or with informed consent and those that the do not. Unfortunately, this goal of risk management currently is seldom achieved.

Structural reliability analysis in Section 4 yields the probability of failure as the quantitative measure of structural performance. In the absence of definitive data on failure costs, the probability of failure also is a surrogate for risk. This estimated failure probability must be compared to a target probability set by regulatory authority or by social custom. The target probability, $\mathrm{P}_{\mathrm{fo}}$, also furnishes one of the constraints in life-cycle cost optimization (see Eqn 5.8), so its determination is a key consideration. There are essentially three points of view on how $P_{f o}$ should be measured (Sorensen, et al, 1994):

(1) Single person acceptable risk: number of accidents or lives lost/number of participants, people exposed;

(2) Society risk: number of accidents or lives lost/number of people in society;

(3) Decision theoretical basis: economic optimum from minimizing the total cost, $C_{i}\left(p_{f}\right)+p_{f} C_{f}$, assuming that $C_{f}$ is independent of $p_{f}$.

Measures (1) and (2) can be quite different, since the relation between individual risk and social risk is nonlinear. Difficulties with measure (3) stemming from an inability to determine $C_{f}$ have been discussed previously.

With a measurement agreed upon, it remains to determine a numerical measure of acceptable risk of $P_{f_{0}}$. Risks associated with engineered structures, while unknown, certainly are very low. It generally is agreed that the target value of $\mathrm{P}_{\mathrm{fo}}$ should depend on the type of structure, the mode of failure, its relative importance to the facility, the residual life desired, and possible socioeconomic consequences of failure. Risks due to structural failures should be much less than risks from operational failures. Structural failures affect many facility systems simultaneously, and thus the consequences are widespread. Moreover, repair of structural components that have failed invariably requires that the facility be taken out of service for an extended period to time while repairs are made. Such downtimes may have severe economic consequences. Beyond these 
general observations, however, obtaining agreement on a precise numerical measure (or range of values) has proved to be elusive.

One approach might be to determine levels of acceptable risk from an examination of existing engineering criteria that have led to facilities that are known to have performed acceptably in the past. Those in support of this so-called calibration approach argue that if the current risks associated with a particular technology were unacceptable, public outrage would have forced corrective action. Indeed, this approach was used to set the load and resistance factors used in the first-generation of probability-based limit states design specifications for ordinary building construction (e.g., LRFD, 1993). The calibration approach works provided that the technology to which the criteria are applied is established, relatively stable and is evolving at a slow enough rate that it is possible to learn from experience. However, historical data on actual failures provide an incomplete picture of acceptable structural risk because structural failure events are so rare. In the case of unique facilities such as offshore platforms and NPPs, historical data on failures are virtually nonexistent. Morover, the calibration approach does not really provide a point estimate of acceptable risk but rather a lower bound, since there is no way of knowing a posteriori whether less conservative criteria (with higher $P_{f_{0}}$ ) would also have proved to be acceptable. In fact, the large range in failure probabilities associated with existing design specifications is evidence to the contrary.

A second approach might be to establish reliability targets by considering comparable and presumably acceptable risks in other human endeavors (Ellingwood, 1994). However, comparisons of structural failure probabilities (assuming that they can be determined) with mortality statistics that have been reported elsewhere are inherently flawed. Such comparisons fail to take into account differences in the population at risk and the large uncertainties associated with reliability evaluation of structures. There currently is no generally accepted mechanism for comparing risks from diverse hazards with low probabilities; nor is there a comprehensive database to support such a comparative risk assessment. Accordingly, it has been suggested that civil engineers should concentrate their efforts on managing hazards rather than on assessment of risks (Comerford and Blockley, 1993).

Determining an acceptable risk for an aging structure presents some unique challenges. It often is not economically feasible to restore an aging facility to its original condition. If the operation of the facility meets an essential overall social objective, such as adequate electric power, the need remains even if the facility is closed and alternatives will be sought. In this case, closing one facility may simply displace the risk to another. There does appear to be some willingness to accept lower reliabilities for older systems and not to require that older systems have the same reliability as new systems.

One can argue that if failure has the same consequences (e.g., involuntary offsite exposure), the risks for an existing facility and a new facility should be the same. However, a sensible definition of risk involves time of exposure as well as level of exposure, and it makes sense to differentiate between a short-term risk and a cumulative risk over an extended service period. The individual risk safety goal (on an annual basis) should be approximately the same for old and new facilities but the cumulative risk for a service period may be different. This is analogous to the difference between hazard $h(t)$ and (cumulative) failure probability, $F(t)$.

Target risks for critical facilities vary widely (Pate-Cornell, 1994). A review of risk targets for NPPs in the US and abroad suggests that the maximum annual probability of severe core damage is on the order of $10^{-4}$; in addition, the maximum tolerable risk offsite individual $=10^{-6} / \mathrm{yr}$ (Okrent, 1987). Core damage probabilities computed from Level 1 PRAs generally are at or below these levels. There is no way of validating such low levels of failure probability conclusively (Lewis, 1985). However, it should be noted that the computed risk of a structure is not an attribute of the structure but of the mathematical reliability model used to analyze the structure. It can be used as an attribute to guide decision-making if the model is dependable (Comerford and Blockley, 1993). Moreover, a comparison of decision alternatives with such risk models used consistently may provide an improved basis for decision-making, irrespective of the absolute risks 
computed. Since this is a temporary answer to a perennial issue, an effort must be made to validate risk models of NPP facilities and to determine their limitations for risk analysis and life extension policies.

For example, Level 1 internal events PRAs of NPPs have been used recently to examine risk due to failure of RPV and piping pressure boundary components, to rank their importance, and to establish inspection priorities (Vo, et al, 1994a, 1994b). ASME Section XI classifications and ISI requirements seem to be in quantitative agreement with rankings based on estimated mean core damage. It was recommended that the probability of core damage due to failures of pressure boundary components be kept to 5\% (or less) of the total core damage probability estimated by the PRA, and that the permitted increase should be allocated among components of a system in accordance with their relative importance. For example, if overall core damage probability is $5 \times 10^{-6}$, pressure-boundary component probability should be $2.5 \times 10^{-7}$, distributed among the components. Components making the greatest contribution to risk should have the most stringent inspection requirements. Steel structural components and systems were not considered in this study, nor were parameter and modeling uncertainties considered explicitly.

\subsection{Summary}

Probability-based criteria for facility risk management are being developed in other industries, most notably in the offshore industry. The American Petroleum Institute (API) is developing new guidelines that can be used for reassessment of offshore platforms (Banon, 1994b). Reassessment is aimed at answering the following questions: Are design/construction drawings available? What is the physical condition of the platform? What were previous inspection results? What level of structural analysis is necessary to assess resistance? Is all existing damage identified and included in the assessment? What are the potential failure modes and consequences? How likely is each failure mode? What level of risk is acceptable? What repair measures are feasible for the damage observed? It seems clear that such generic questions are germane to the evaluation of aging in NPPs as well. In the nuclear industry, ASME has formed a research group to consider risk-based inspection guidelines (ASME, 1992). In related research (Vo, 1994b), intemal events Level 1 (core damage) PRAs were used to identify the most significant pressurized systems for plant risk and therefore for special attention during inspection. Seven PWRs and two BWRs were considered. Passive structural components were not considered.

The data to support risk-based inspection/maintenance programs for steel containments and liners are not complete at present and will be developed in a later phase of this research program. Nondestructive evaluation provides solutions to the lack of quantitative data. If the NDE information (generally at the local scale) can be incorporated into a rational structural condition and reliability assessment, the long-term economic benefits will be significant. 


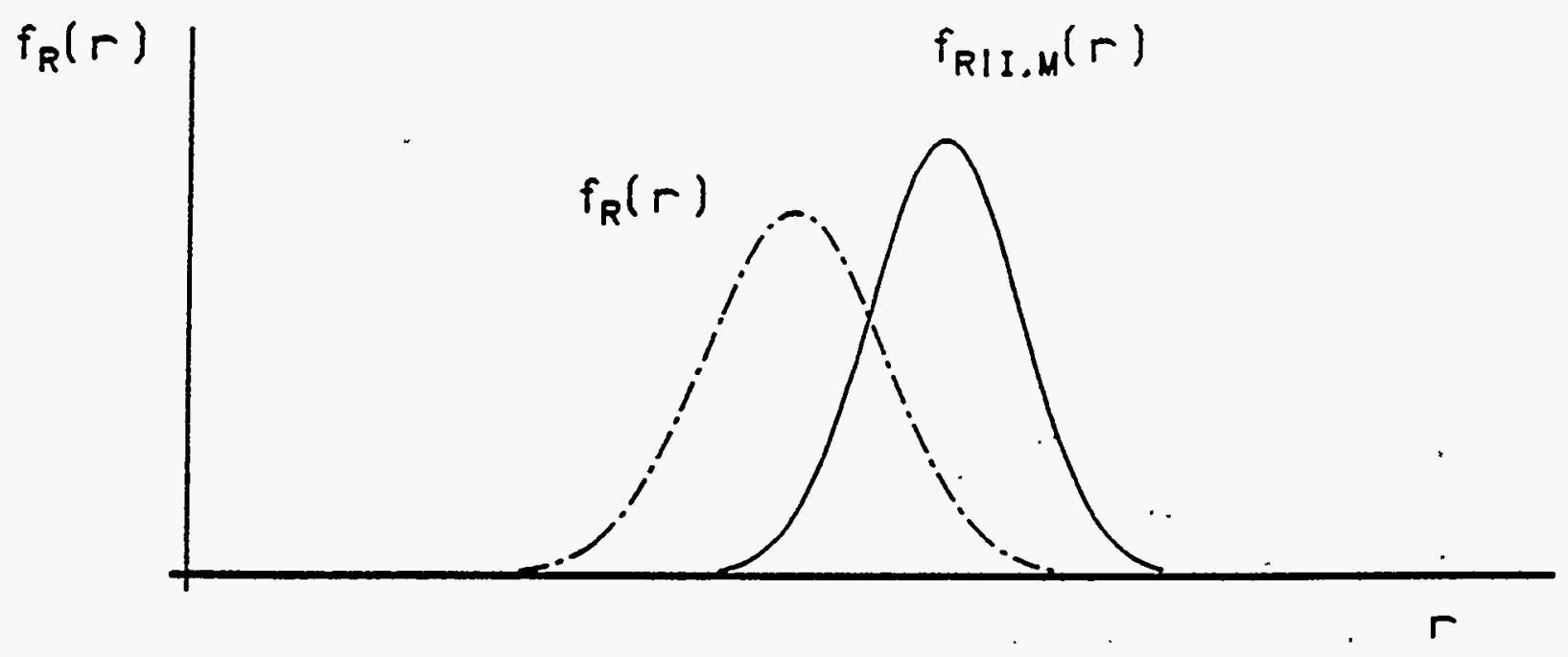

Figure 5.1 - Bayesian updating of resistance

NUREG/CR-5442

70 


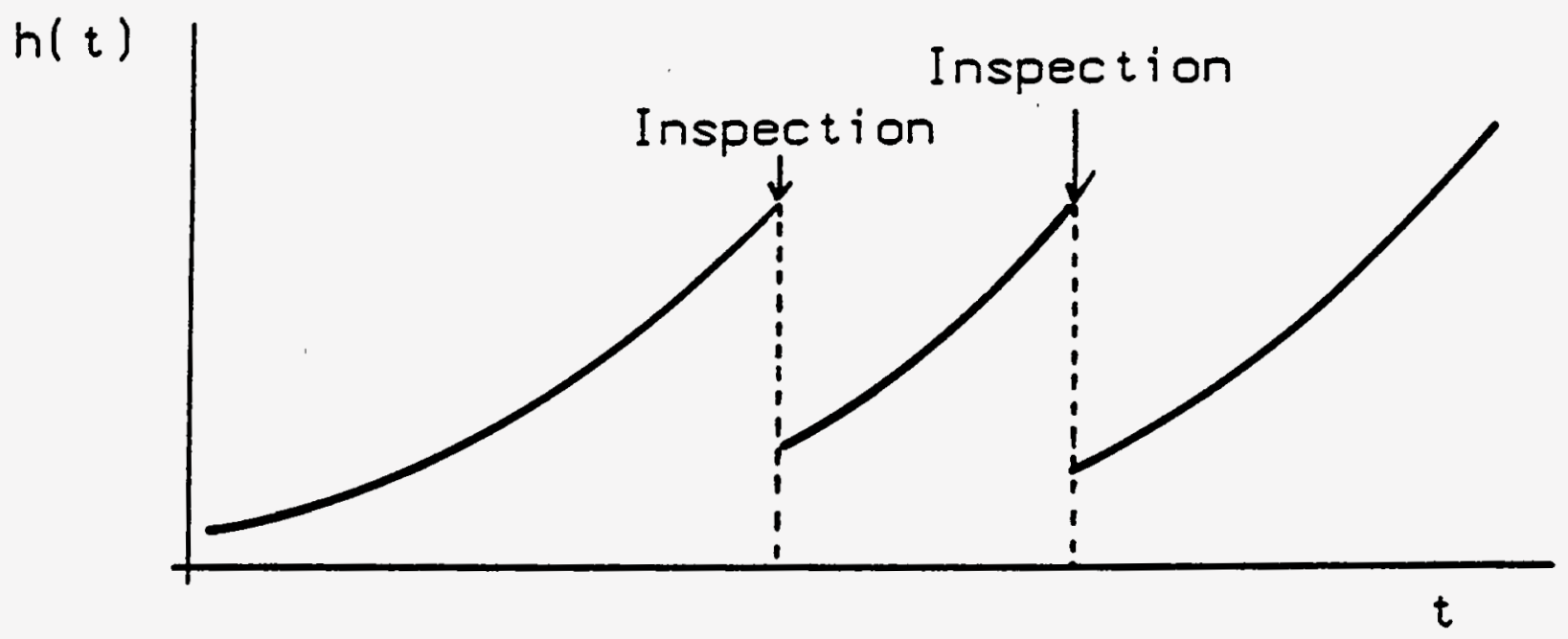

Figure 5.2 - Effect of in-service inspection and maintenance on $h(t)$ 


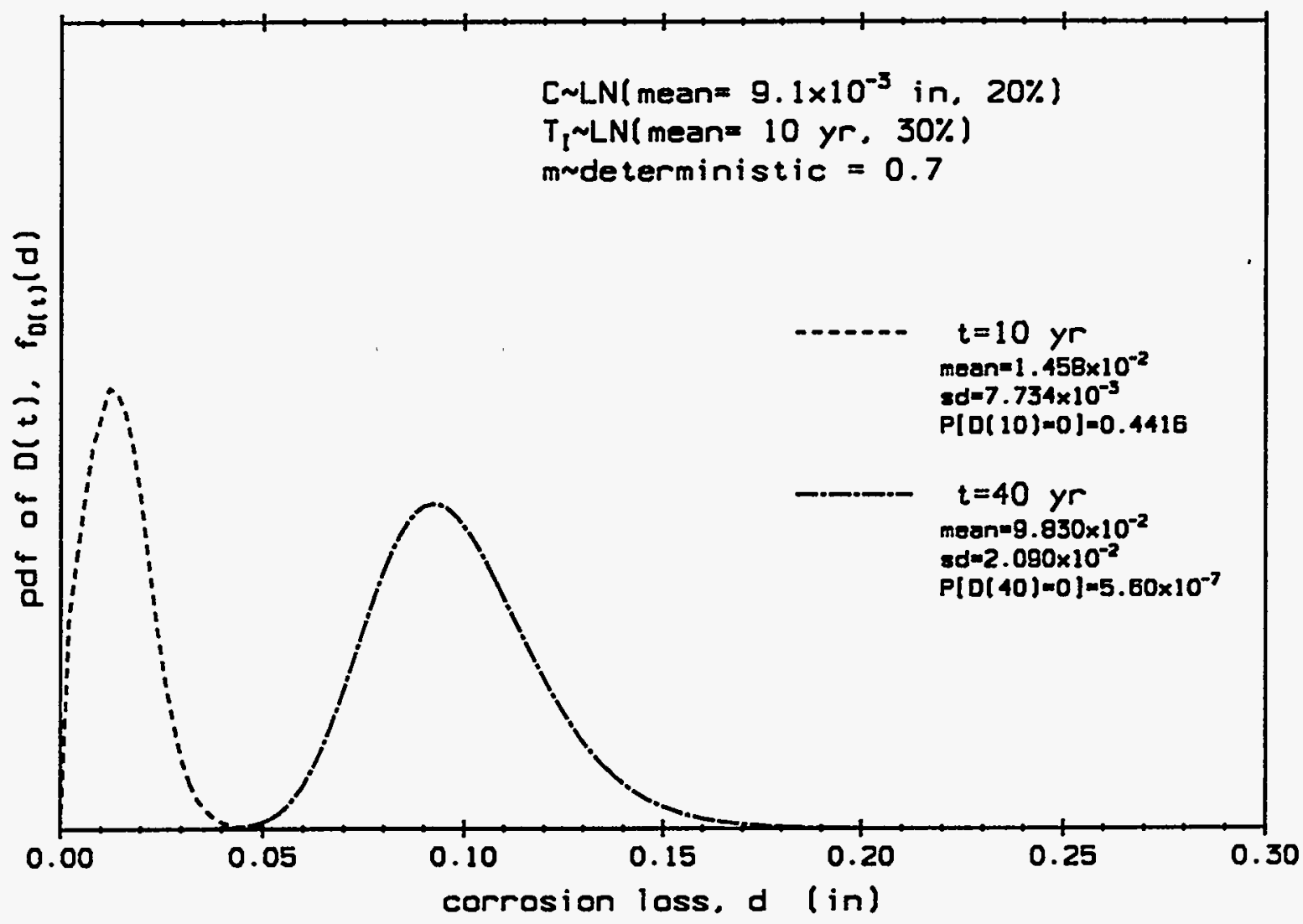

Figure 5.3 - CDF of corrosion depth at 10 and 40 years 


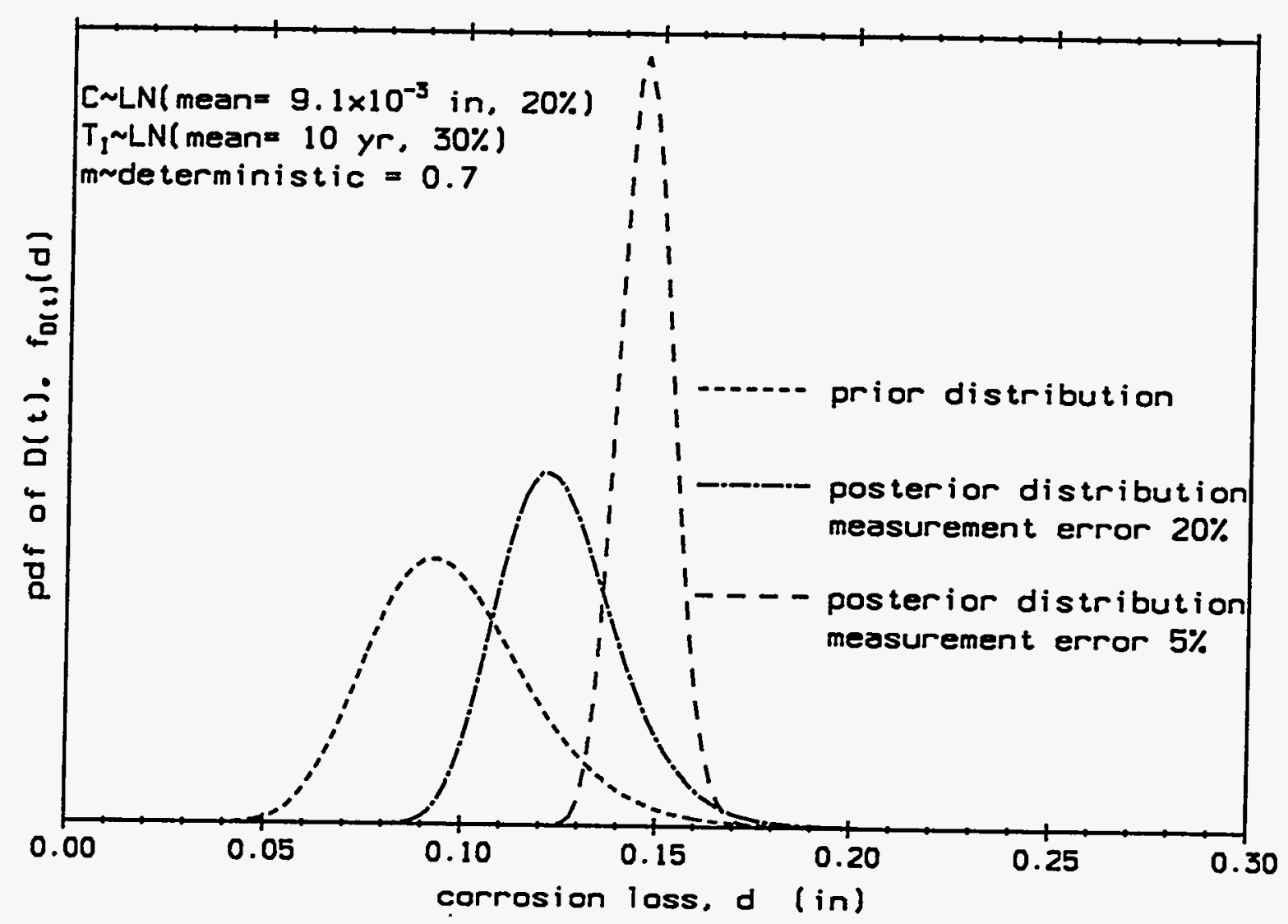

Figure 5.4 - CDF of corrosion depth, updated following inspection 


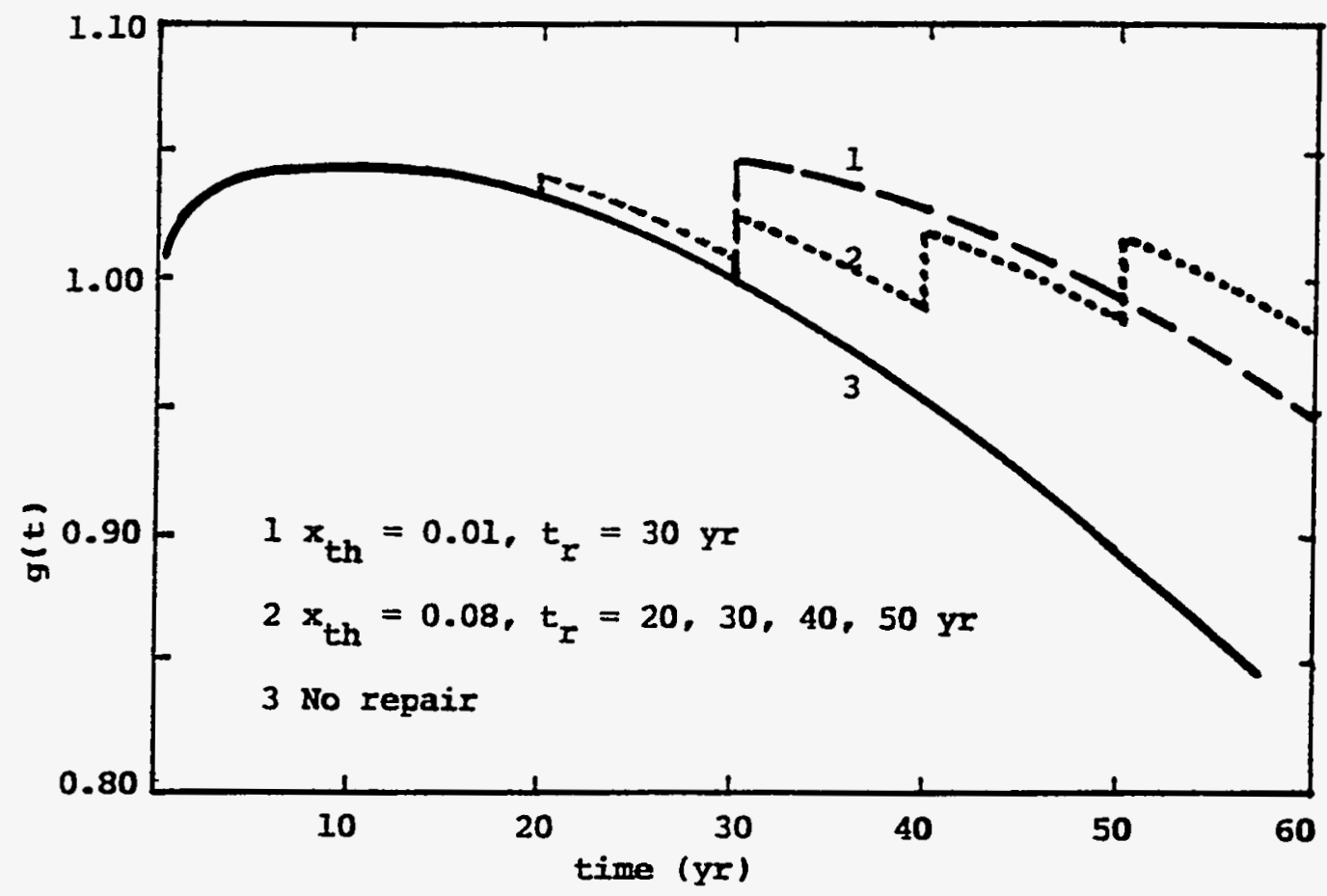

Mean degradation functions for slab under alternate ISI/M policies

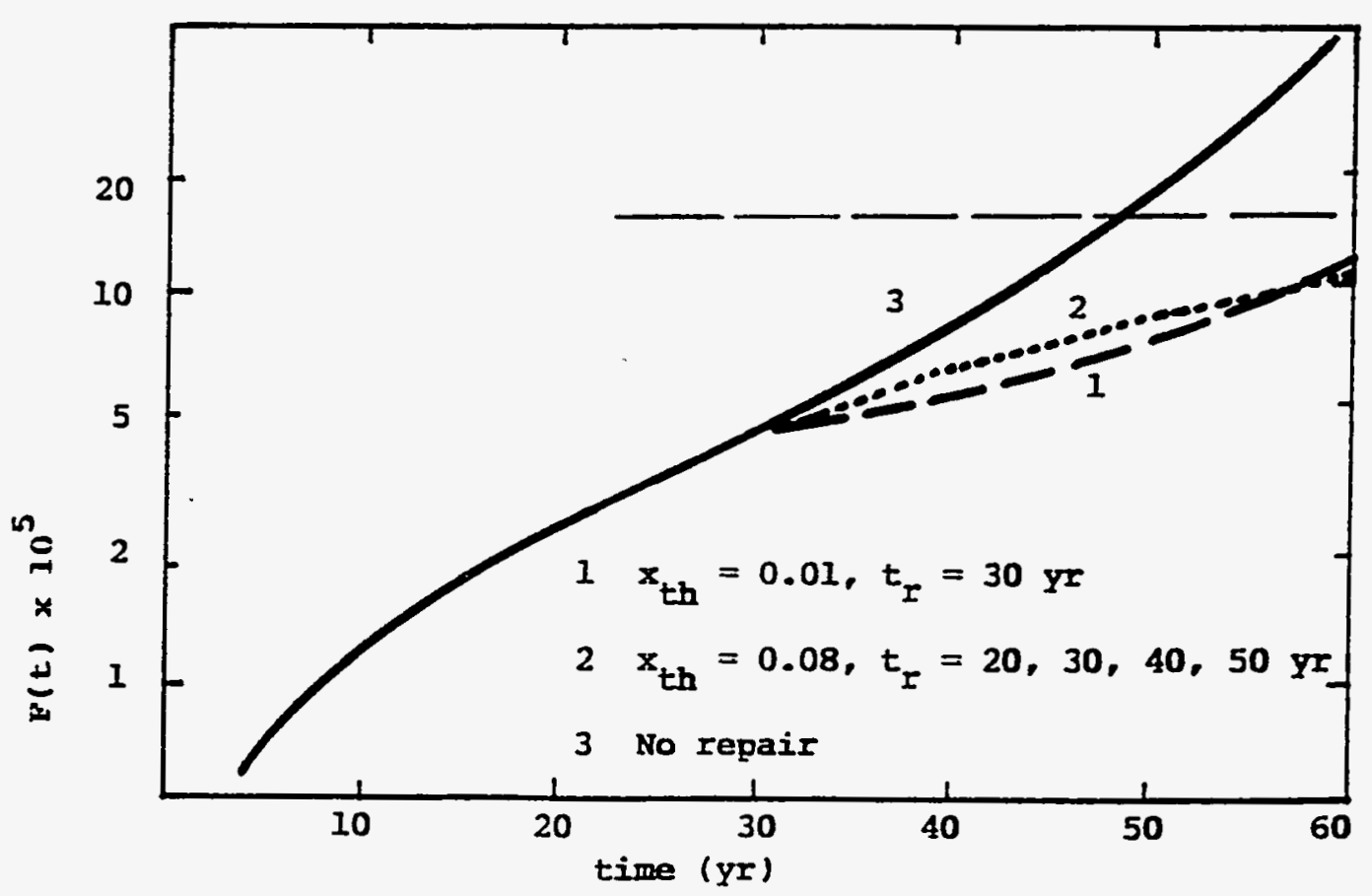

Failure probability of slab for alternate ISI/M policies

Figure 5.5 - Reliability for alternate ISI/M policies 


\section{MARKOV PROCESS MODEL OF DAMAGE ACCUMULATION}

Damage accumulation in a large complex structure may be very difficult to track. The progression of damage accumulation in a structure can be modeled as a Markov process (Bogdanoff and Kozin, 1985; Rahman and Grigoriu, 1993). The Markov model does not provide any insight into the mechanics of damage accumulation. It simply provides a convenient framework for describing the evolution of damage state probabilities over time and, with its matrix formulation, provides a convenient algorithm for computerization. The Markov formulation can be viewed as operating on a number of levels. At the simplest level, it can be used to track "damage" when damage and effectiveness of in-service inspection and maintenance can only be described linguistically. At the other extreme, the state of damage can be determined probabilistically using stochastic computational mechanics formulations developed in the previous sections of this report.

We begin by envisioning a "duty cycle" (DC) for the structural system, which is some repetitive sequence of loads during the service life that arises from the operation of the facility and during which damage can accumulate. At one extreme, loads from a standard cycle between refueling outages might be one example; at the other extreme, in the case of a broad-band load history, each distinct load might be considered a DC. The Markov model arises from the idea that the increment of damage that accumulates during each DC depends only on the value of damage that has accumulated up to the start of that DC; the damage increment is statistically independent of the process by which damage accumulated prior to the start of the DC. Under these conditions, damage accumulation can be modeled as a Markov process, with time measured in DC units. If the damage state is discretized as well, the process is referred to as a Markov chain.

As a simple illustration of this concept, we might envision the structure to be in one of $i=1,2, \ldots, M$ discrete states of damage, $D(t)=i$ at any time $t$ (note that $t$ can be an integer if time is measured in $D C$ units or in cycles). The set $\{1,2 \ldots, \mathrm{m}\}$ is denoted the state space, and can be written for a structural component or system. One might say that the structure is undamaged if $D(t)=1$ and that the structure has failed if $D(t)=$ $M$. These definitions are completely arbitrary; the damage measure could be normalized as $D(t)=(i-1) /(M-1)$ so that $\mathrm{D}(\mathrm{t})$ is consistent with the previous discussion of damage accumulation by the Palmgren-Miner hypothesis. The probability that the structure is in damage state, $i$, at time $t$ is denoted,

$$
P[D(t)=i]=P_{i}(t) ; i=1,2, \ldots, M
$$

The prior service history of structural behavior up to time $t$ (here, $t$ could be discrete or continuous) can be expressed as the collection of observation times and damage states,

$$
H_{t}=\{D(0), D(1), \ldots, D(t-1)\}
$$

The Markov property implies that the conditional probability that $D(t)=i$, conditioned on prior service history, is,

$$
P\left[D(t)=i \mid H_{t}\right]=P[D(t)=i l D(t-1)=j]
$$

in which,

$$
\sum_{i=1}^{M} P[D(t)=i \mid D(t-1)=j]=1.0 ; j=1,2, \ldots, M
$$

In other words, if the immediate state of the structure is known, the future state is independent of past states. 
At some later time, $v$, the probability that the structure is in state $j$ is,

$$
P[D(v)=j]=\sum_{i=1}^{M} P_{i j}(v \mid t) P_{i}(t)
$$

in which $P_{i j}(v / t)=P[D(v)=j i D(t)=i]$ is the conditional probability that the structure is in state $j$ at $v$, given that it was in state $i$ at $t$. Eqn 6.4 is simply a statement of the theorem of total probability. Considering all $M$ damage states and collecting elements $P_{i}(t)$ into column vector, $P(t)$, Eqn 6.5 can be expressed in matrix form:

$$
P(v)=P(v l t) P(t)
$$

in which $\mathrm{P}(\mathrm{v} / \mathrm{t})$ is denoted the "transition probability matrix" between stages $t$ and $v$. With the Markov property, the elements of the transition probability in this summation are (the Chapman-Kolmogorov equation),

$$
P[D(v)=j \mid D(t)=i]=\sum_{k=1}^{M} P_{i k}(v \mid u) P_{k j}(u \mid t)
$$

for $\mathrm{t}<\mathrm{u}<\mathrm{v}$, and are independent of the history of damage accumulation prior to $t$.

According to Eqn 6.6, matrix $\mathrm{P}(\mathrm{v} / \mathrm{t})$ can be constructed as the product of one-step transition matrices, $\mathrm{T}(\mathrm{k})$ :

$$
P(v \mid t)=\prod_{k=0}^{t} T(v-k)
$$

Element $p_{M M}$ of $T(v-k)$ is unity, the absorbing state of the chain (representing failure). In general, $T(v-k)$ is a function of time (or index, $k$ ). However, in the case where $P(v / t)$ depends only on $v-t$, the chain is said to be homogeneous (or have stationary increments), and

$$
P(v \mid t)=T^{(v-1)}
$$

in which $\mathrm{T}=$ stationary one-step transition probability matrix. Much of the mathematical literature on Markov chains has been developed for processes with stationary increments. It should be noted that a process with stationary increments is not necessarily a stationary process, i.e., one in which the probability function $\mathrm{P}[\mathrm{D}(\mathrm{t})$ $=\mathrm{i}]$ is invariant in time.

One can use Eqn 6.7 to track the evolution of damage accumulation through the service life of a structure, provided that the initial damage state vector, $\mathrm{P}(0)$, is known. In general, we would have,

$$
P(t)=\underset{k=0}{t} T(t-k) P(0)
$$

If the structure is undamaged in its initial condition, $P(0)=(1,0, \ldots, 0)^{t}$. Since matrix multiplication is not commutative, the order of damage cycles is important for damage accumulation. If the matrix $T(t-k)$ is independent of $\mathrm{k}$ and the process has stationary increments, then

$$
P(t)=T^{t} P(0)
$$

The elements of the transition matrix can be estimated by modeling the structural deterioration mechanisms described in Section 3 as stochastic processes. The nature of structural degradation determines the characteristics of $\mathrm{T}$. Since damage accumulation is assumed to be irreversible, $\mathrm{T}$ is a lower triangular matrix in the absence of any in-service maintenance, repair, or other human intervention. If damage growth is gradual or if the time interval is small, only small changes of state are possible, and $\mathrm{T}$ is strongly diagonal. In the limiting case when damage accumulation can only increase by one state during a DC, T has only one diagonal and one off-diagonal term per row or column. 
The issue of whether the damage accumulation can be modeled as a process with stationary increments (with $\mathrm{T}$ independent of time) must be addressed in the context of the common damage accumulation mechanisms and their mathematical modeling. For example, in the case of stable propagation of a crack in a weldment exposed to a benign environment, the incremental crack growth during the application of the $(i+1)$ th load in a sequence of statistically independent loads can be determined, approximately, from

$$
\Delta a_{i+1}=C\left(Y \Delta S_{i+1}\right)^{m}\left(\pi a_{i}\right)^{m / 2}
$$

It is clear that $\Delta \mathrm{a}_{\mathrm{i}+1}$ is a function of the existing crack size, $\mathrm{a}_{\mathrm{i}}$, but the prior history of damage accumulation leading to $\mathrm{a}_{\mathrm{i}}$ does not affect $\Delta \mathrm{a}_{\mathrm{i+1}}$. Thus, damage accumulation manifested by $\mathrm{a}_{\mathrm{i}}$ could be modeled as a Markov process, and $\mathrm{T}$ should not depend on time. On the other hand, it is not obvious that the damage increments should be stationary in the presence of a degrading structure. If the weldment were exposed to an aggressive environmental factor, $\theta(t)$, that caused cumulative time-dependent metallurgical changes leading to increased embrittlement, it seems apparent that $\Delta a_{i+1}$ due to $S_{i+1}$ would depend not only on $a_{i}$ but on $\theta(t)$ as well. If $\theta(t)$ is constant or varies slowly in time, $T$ would depend on the age at which $S_{i+1}$ occurred, i.e., other factors being equal, $\Delta \mathrm{a}_{\mathrm{i}+1}$ certainly would be larger if the exposure time were increased. If $\theta(t)$ fluctuated significantly in time, it is conceivable that crack growth could no longer be modeled as a Markov process.

If in-service inspection and/or maintenance is performed, the results can be incorporated in the damage evaluation process. As discussed in Section 5 , there can be various levels of inspection, maintenance and repair or replacement. It is assumed that the duration of in-service inspection/maintenance is very short with respect to the service life.

When the structure is inspected at time $r$, something is learned about how damage has actually evolved in time. However, no inspection is perfect. There is uncertainty in the ability of any NDE method to detect a defect and, if detected, to size the defect appropriately. The mathematical model of damage leading to (prior) estimate $D(t)$ also is uncertain. This uncertainty can be expressed by an NDE matrix, $E$, with elements $\mathrm{e}_{\mathrm{ij}}$ defined as,

$$
e_{i j}=P\left[D_{o b s}(r)=i \mid D(r)=j\right]
$$

in which $\mathrm{D}_{\mathrm{obs}}(r)$ is the observed state of damage and $\mathrm{D}(r)$ is the prior (predicted) state. If the mathematical model of damage evolution is accurate and in-service NDE is perfect, $E$ is a unit matrix; otherwise, $E$ has nonzero off-diagonal elements, reflecting uncertainties in modeling and NDE. The elements $e_{i j}$ of $E$ can be determined by Bayesian methods, dependent on the data collected during in-service inspection. As part of this process, it may facilitate analysis to break the determination of $E$ down into separate steps, depending on accuracy of damage accumulation analysis, NDE, extent of structure inspected, and so forth. This more refined option will not be pursued at present for simplicity in presenting the overall concept.

Associated with the results of the in-service inspection is an "action" or decision space consisting of $\mathrm{K}$ possible actions. This action space is denoted,

$$
A=\left\{a_{1}, a_{2}, \ldots, a_{k}\right\}
$$

in which $\mathrm{a}_{\mathrm{i}}=$ particular action. The space of actions may be as simple as \{do nothing, repair, replace\}, or may be more complex, depending on the requirements of the problem. The action taken at the time of inspection depends on what is learned about the structure at that time, i.e., from the observations during the inspection. This can be expressed in probabilistic terms by a policy matrix, $B$, with elements $b_{\mathrm{ij}}$ defined by the conditional probabilities, 


$$
b_{i j}=P\left[A=a_{i}\left[D_{o b s}(r)=j\right]\right.
$$

If no damage was found, no maintenance or repair action would be taken. Typical elements of B might be:

$$
\begin{aligned}
& \mathrm{P}\left[\text { repair } \mathrm{D}_{\text {obs }}(\mathrm{r})=0\right]=0 \\
& \mathrm{P}\left[\text { replace } \mathrm{D}_{\mathrm{obs}}(\mathrm{r})>0.5\right]=1.0
\end{aligned}
$$

and so forth. The policy matrix generally would be nonstationary; if, for example, if the remaining service period was short, one probably would not replace or repair the component unless $D(r)$ were relatively large.

Finally, one must consider the effectiveness of the action taken in mitigating damage accumulation. The effectiveness of repair can be modeled by the consequence matrix, $C$, defined at time $t>r$, ( $t-r$ is small), with elements $c_{i j}$ defined by the conditional probabilities,

$$
c_{k j}=P\left[D(t)=k \mid A=a_{i} n D_{o b s}(r)=j\right]
$$

The consequence matrix reflects the fact that maintenance and repair operations are not perfect; indeed, certain repairs, such as rewelding, can actually accelerate damage growth if not done properly. Typical elements of C might be,

$$
\begin{aligned}
& P[D(t)=0.01 A=\text { replace } n D(r)=0.5]=0.99 \\
& P[D(t)=0.01 A=\text { repair } n D(r)=0.5]=0.95
\end{aligned}
$$

and so forth.

Combining these steps of inspection and repair (together or separately), their overall effectiveness in mitigating the damage states can be modeled by,

$$
P(t)=C B E P(r)
$$

in which $P(r)=$ state vector of damage prior to inspection at $r, P(t)=$ state vector following repair at time $t$ shortly after $r$, and the remaining matrices represent the effectiveness of in-service inspection and repair as defined above. All human interaction with the structure and its behavior is encapsulated in matrices C, B and E.

When the necessary data to model stochastic deterioration quantitatively are unavailable, a simpler approach can be taken, in which the states of damage are described linguistically. For example, a simple fivestate representation might be,

\begin{tabular}{ll} 
Damage State & D \\
\hline Undamaged & 0.00 \\
Minor & 0.25 \\
Moderate & 0.50 \\
Major & 0.75 \\
Unacceptable & 1.00
\end{tabular}


The transition probabilities would be estimated subjectively in this situation from the results of in-service inspection. Agreement on what is, e.g., "minor" damage; would be necessary in order to use the model to support a rational aging management strategy.

\section{Illustration}

To illustrate the above concepts in as simple way as possible, consider a structural system that is subjected to some unspecified degradation action. The state of the structural system can be described by the five states in the above table. We will use the Markov model to view the progression of damage in increments of 10 years over a service life of 40 years and to determine the effectiveness of in-service inspection.

. . : The initial state of the structure is described stochastically by the probability mass function (PMF) $P(0)$, which depends on the quality assurance and control programs in place at the time of design and construction. In this illustration, we will consider two different initial state vectors $P(0)$ :

(1) $P(0)=(1,0,0,0,0)^{t}$

(2) $P(0)=(0.95,0.049,0.001,0,0)^{t}$

The first corresponds to high-quality construction; the probability of any inherent initial damage is assumed to be zero. The second models construction with poor quality control.

In concept, the progression of damage in 10-year increments of time can be evaluated using the timedependent reliability principles outlined in Section 4 , and elements of the transition probability matrix, $T$ (cf Eqn 6.8) can be determined accordingly. The transition probability matrix for each 10 -yr increment is assumed to be stationary in time; that is, the probability law describing damage growth in 10 years depends only on the damage state at the beginning of the 10 -yr period, not on the previous history. For illustrative purposes, this transition probability matrix is assumed to be:

$\mathrm{T}=\begin{array}{lllll}0.99 & 0 & 0 & 0 & 0 \\ 0.009 & 0.99 & 0 & 0 & 0 \\ 0.001 & 0.009 & 0.98 & 0 & 0 \\ 0 & 0.001 & 0.015 & 0.95 & 0 \\ 0 & 0 & 0.005 & 0.05 & 1\end{array}$

The probability mass function describing the state of damage after 40 years can be evaluated from Eqn 6.10, in which

$\begin{array}{lllll}0.96059 & 0.0 & 0.0 & 0.0 & 0.0 \\ 0.03489 & 0.96059 & 0.0 & 0.0 & 0.0 \\ \mathrm{~T}^{40}=.00439 & 0.03438 & 0.92237 & 0.0 & 0.0 \\ 0.00011 & 0.00444 & 0.05393 & 0.81451 & 0.0 \\ 0.00002 & 0.00051 & 0.02370 & 0.18549 & 1.0\end{array}$

Substituting the two initial vectors $\mathrm{P}(0)$ into Eqn 6.10 , we obtain:

(1) $\mathrm{P}(40)=(.96059, .03489, .00439, .00011, .00002)^{\mathrm{t}}$

(2) $\mathrm{P}(40)=(.91256, .08021, .00678, .00038, .00007)^{t}$ 
where the time is understood to be prior to any ISI/M. Note that the impact of shoddy design/construction practice is to shift the probabilities toward the higher states of damage after 40 years.

An inspection now is performed at 40 years. The uncertainty in this inspection process is encapsulated in matrix $E$ (Eqn 6.12). The elements of this matrix can be determined using the NDE methodology described in Sections 3 and 5. Here, we assume that:

$\mathrm{E}=\begin{array}{lllll}0.95 & 0.025 & 0 & 0 & 0 \\ 0.05 & 0.95 & 0.025 & 0 & 0 \\ 0 & 0.025 & 0.95 & 0.025 & 0 \\ 0 & 0 & 0.025 & 0.95 & 0.05 \\ 0 & 0 & 0 & 0.025 & 0.95\end{array}$

The high probabilities on the diagonal indicate that in $95 \%$ of the evaluations performed, the "observed" damage is the same as the "actual" damage; however, there is a margin for error in interpretation, measured by the small off-diagonal probabilities. This margin is directly related to the sizing errors for a particular NDE method, as illustrated in Figures 3.6 - 3.7. The probability of the observed damage state for Case (1) from Eqn 6.15 is,

(1) $\mathrm{P}\left(\mathrm{D}_{\text {obs }}\right)=(.91343, .08128, .00505, .00022, .00002)^{\mathrm{t}}$

(2) $P\left(D_{\text {obs }}\right)=(.86894, .12200, .00846, .00053, .00007)^{t}$

Note that the observation error in this case tends to shift the observed damage states toward more conservative values.

The actions taken upon inspection are based on the states of damage that are observed. These actions are determined by the inspection policy imposed by the facility owner or its regulator. Any one of several actions may be taken, as indicated in Eqns 6.13. Suppose that the following deterministic policy is adopted:

$$
\begin{array}{ll}
D_{\text {obs }}(r)=\text { undamaged: } & A=\text { do nothing } \\
D_{\text {obs }}(r)=\text { minor: } & A=\text { repair } \\
D_{\text {obs }}(r)=\text { moderate: } & A=\text { repair } \\
D_{\text {obs }}(r)=\text { major: } & A=\text { replace } \\
D_{\text {obs }}(r)=\text { unacceptable: } & A=\text { replace }
\end{array}
$$

The effectiveness of repair must be considered in developing terms in the consequence matrix, C, in Eqn 6.14.

For illustration,

$\mathrm{C}=\begin{array}{lllll}0.999 & 0.995 & 0.995 & 0.995 & 0.995 \\ 0.001 & 0.003 & 0.003 & 0.005 & 0.005 \\ 0 & 0.002 & 0.002 & 0 & 0 \\ 0 & 0 & 0 & 0 & 0 \\ 0 & 0 & 0 & 0 & 0\end{array}$

For example, the last two columns imply that if the component is severely or unacceptably damaged and is replaced, the probability is $99.5 \%$ that following repair it will be "good-as-new," but there is a $0.5 \%$ probability that the replacement will not be fully effective. Similarly (column 2), if the damage is minor and is repaired, there is a $0.3 \%$ probability that the repair is ineffective, and $0.2 \%$ probability that it actually worsens the 
condition of the component. Elements of matrix $\mathrm{C}$ would have to be determined from an examination of the effectiveness of various in-service inspection and repair policies.

Finally, the damage state vector following inspection and repair is determined from Eqn 6.15 . For the two cases, we have:

$$
\begin{aligned}
& \text { (1) } \mathrm{P}(40)=(.99865, .00118, .00017,0,0)^{t} \\
& \text { (2) } \mathrm{P}(40)=(.99848, .00126, .00026,0,0)^{t}
\end{aligned}
$$

in which the time is immediately following ISI/M. Appropriate in-service inspection and maintenance policies can be determined by selecting those policies that keep the probabilities of moderate (or greater) damage acceptably small. Note that if one starts off with a poorly designed and constructed structure (Case 2), this may be practically impossible to accomplish.

Additional research is required to relate the elements of the transition probability and ISI/M matrices to the stochastic mechanics of structural degradation and the uncertainties in common NDE procedures. However, the formalism of the Markov model provides a simple and convenient way to visualize damage accumulation in a NPP structure over its service life or a service life extension. It is recommended that this approach be developed further as a tool for facility life extension evaluations. 
$-1$ 


\section{RECOMMENDATIONS FOR FURTHER WORK}

This report has reviewed published research on mechanisms of structural deterioration caused by operation and aggressive environmental effects and on time-dependent reliability methods that can be used to perform condition assessments of steel containments and liners in nuclear power plants. Reliability-based methodologies and data currently are at a state where it should be possible to develop and institute risk-based in-service inspection and maintenance policies for NPP facilities during the next several years. The methodology leading to this policy should be relatively simple and should be consistent with construction and in-service inspection and maintenance databases currently maintained by the utilities and by the NRC.

Risk management policies should be developed by starting at the system level with a qualitative requirement, followed by a quantitative criterion for acceptable performance expressed in probabilistic terms. Subsequent criteria to meet the system criterion should be addressed at the component level. Specific research needs have been collected into five groups below.

(1) Identification of degradation mechanisms and models

It is believed that most relevant degradation mechanisms have been identified in the current report. A survey of NPP operators should be conducted to obtain a sense of the relative importance of the mechanisms identified, in terms of structural behavior, relative likelihood and economic impact on facility performance.

(2) Time-dependent reliability analysis procedures

The response of a steel containment or liner structure to combinations of operating loads, self-straining thermal effects, and accidental loads is complex and unavailable in closed form. Nonlinear finite element analysis is required to determine structural response due to these effects. Response surface techniques can be used along with FEA to construct sufficiently accurate limit state models to perform reliability calculations of degraded containments.

Methods to analyze reliability of steel containments and liners subjected to combinations of fatigue and corrosion must be developed. In particular, little research is available on probabilistic aspects of corrosion, and techniques need to be developed for this purpose, requiring stochastic modeling of the initiation and active growth phases of corrosion discussed in Section 2. Existing probabilistic fatigue/fracture analysis methods must be adapted to consider the unique loading cases and environments found in steel containments and liners. Synergistic effects typical of corrosion/fatigue require further study.

Evaluation of damage accumulation through principles of damage mechanics shows great promise. Damage mechanics permits in-service assessment of residual strength or safety margins in situations where there may be no visible manifestation of damage that can be readily be detected by the usual NDE methods described in Section 3. Such situations would include: damage due to gross inelastic deformation; fatigue damage prior to formation of a detectable crack; elevated temperature creep; and metallurgical embrittlement due to irradiation. In addition, the damage mechanics formalism permits a variety of damage accumulation mechanisms to be evaluated by the same basic fundamental thermodynamics principles. Damage mechanics theory indicates that the state of damage can be related directly to the modulus of elasticity, which can be measured or inferred from stress-wave or other easily performed tests.

(3) Data collection and evaluation

Research to obtain data to characterize aging in structural materials based on accelerated aging tests has shown that such data may be unreliable when extrapolated to field conditions. Field surveys and in-situ 
measurements of aging structures are required to identify the necessary descriptive parameters and to provide recommendations for any subsequent data collection in a later phase of the methodology development.

(4) Reliability measures and targets

One of the ingredients of a reliability-based condition assessment and service life prediction is the notion of an acceptable risk or acceptable limit state probability. The selection and interpretation of such quantitative reliability measures is difficult. Better estimates are required to support decision-making. In the presence of limited data and little opportunity to validate risk analysis methods, any risk measure is more useful in a comparative sense than as an absolute target. Research is required to investigate the feasibility of developing a framework for comparative risk assessment. This effort seems particularly important in view of the public nature and visibility of NPP facilities. Alternate maintenance strategies can be evaluated and compared using probabilistic methods where the uncertainties can be dealt with explicitly and systematically. This method of comparative evaluation provides an audit trail for decision-making, which can be revised in a rational fashion if additional information subsequently becomes available.

The use of fragility modeling of steel containments and liners as an adjunct to risk management of NPP facilities should be investigated further. A fragility analysis effectively uncouples the probabilistic analysis of system performance from the analysis of the natural or man-made hazard. Focussing on the component or system fragility allows the facility manager insight regarding the dominant contributors to risk without the need to resolve issues associated with the hazard determination, many of which are difficult or controversial and most of which are accompanied with high levels of uncertainty.

(5) Facility management policies

The reliability-based methodology can be used as a basis for developing rational in-service inspection, evaluation, and maintenance programs. However, the reliability methods are numerically intensive and complex and may be difficult to apply on a case-by-case basis. Accordingly, a set of requalification guidelines should be developed for in-service condition assessment. These guidelines should be reliability-based, but couched in a form that would be relatively easy to use. The guidelines would address the following specific issues:

What inspections should be conducted?

What additional analyses should be performed? Can they be simple or must they be complex? Should they be based on linear elastic analysis or nonlinear analysis? Can they be limited to static behavior, or must dynamic effects be considered explicitly

Should the requalification be done in terms of old or new structural codes?

What inspection and repair measures are consistent with performance objectives, acceptable risk or reliability, and cost?

What sort of documentation should be required?

It is important that the requalification guidelines be made understandable for field engineers. Communication, feedback and control (adaptive learning) are essential ingredients of risk management of facilities that evolve in time. Efforts should be made to formalize these processes so as to minimize the realtime learning process for NPP facilities. 


\section{REFERENCES}

ACI (1985). " "Code requirements for nuclear safety related concrete structures (ACI 349-1985). " American Concrete Institute, Detroit, MI.

ACI (1989). "Building code requirements for reinforced concrete (ACI 318-1989)." American Concrete Institute, Detroit, MI.

Ahammed, M. and Melchers, R.E. (1995). "Probabilistic analysis of pipelines subjected to pitting corrosion leaks." Engineering Structures, vol 17, no. 2, pp. 74-80.

Akashi, M., Fukuda T. and Yoneyama, H. (1990). "A corrosion localization assessment of the mild steel used for nuclear waste package." Mat. Res. Soc. Symp. Proc. vol. 176.

ASME Research task force on risk-based inspection guidelines (1992). "Risk-based inspection - development of guidelines." NUREG/GR-0005 (Vols. 1 and 2), US Nuclear Regulatory Commission, Washington, DC.

ASTM G 101-89. "Standard guide for estimating atmospheric corrosion resistance of low-alloy steels." Am. Soc. For Testing and Mat., Philadelphia, PA.

Aziz, P.M. (1956). "Application of the statistical theory of extreme values to the analysis of maximum pit depth data for aluminum." Corrosion, NACE, 12, (10), pp 495t-506t.

Banon, H., et al (1994a). "Assessing fitness for purpose of offshore platforms. I: Analytical methods and inspections." J. Struct. Engrg. ASCE 120(12):3595-3612.

Banon, H. (1994b). "Assessing fitness for purpose of offshore platforms. II: Risk management, maintenance and repair." J. Struct. Engrg. ASCE 120(12):3613-3633.

Barsom, J. and Rolfe, S. (1987). Fatigue and fracture control in structures. Prentice-Hall, Englewood Cliffs, NJ.

Berens, A.P. (1989), "NDE reliability analysis," Metals Handbook, Vol. 17, ASM international, Metals Park, Ohio, 689-701, 1989.

Berger, D.M. (1983). "Fundamentals and prevention of metallic corrosion." Corrosion and Corrosion Protection Handbook, Marcel Dekker, New York.

Bjerager, P. (1990). "On computation methods for structural reliability analysis." Struct. Safety, 9(2): 79-96.

Bogdanoff, J. and Kozin, F. (1985). Probabilistic models of damage accumulation. Wiley-Interscience, New York, NY.

Bowen, W.M., Heasler, P.G. and White, R.B. (1989). "Evaluation of sampling plans for in-service inspection of steam generator tubes." NUREG/CR-5161, U.S. Nuclear Regulatory Commission, Washington, DC.

Bray, D.E. and Stanley, R.K. (1989). Nondestructive evaluation. McGraw-Hill, New York, NY.

Broek, D. (1988). The practical use of fracture mechanics. Dortrecht; Kluwer Academic Publishers, The Netherlands. 
Bucher, C. and Bourgund, U. (1990). "A fast and efficient response surface approach for structural reliability problems." Struct. Safety. 7(1):57-66.

Casciati, F., Colombi, P. and Faravelli, L. (1993). "Lifetime prediction of fatigue sensitive structural elements." Struct. Safety 12(2):105-111.

Casciati, F., and Colombi, P. (1993). "Load combination and fatigue reliability problems." Struct. Safety 13(1):93-112.

Chaboche, J.L. (1988). "Continuum damage mechanics, Parts I and II." I. App. Mech. ASME 55(3):59-71.

Chase, S.B. (1994). "A New Fatigue Crack Detection System." Structural Materials Technology, (Scancella et al, ed.), Atlantic City, New Jersey, pp 334-338, 1994.

Cherry, J. L. (1995). "Interim report on capacity of degraded containments." Sandia National Laboratories, Albuquerque, NM.

Chopra, O.K., Shack, W.J. and Rosinski, S.T. (1991). "Radiation embrittlement of the neutron shield tank from the Shippingport Reactor." NUREG/CR-5748, Washington, DC.

Chung, H. M., et al (1993). "Environmentally assisted cracking in light water reactors." NUREG/CR-4667 (16 Volumes), Washington, DC.

CIB (1987). "Prediction of service life of building materials and components.: CIB W80/RILEM 71-SPL, Materials and Structures

20:55-77.

Clifton, J.R. (1993). "Predicting Service life of concrete." J. Materials. ACI 90(6):611-617.

Comerford, J.B. and Blockley, D. I. (1993). "Managing safety and hazard through dependability." Struct. Safety 12(1):21-33.

Committee on Fatigue and Fracture Reliability (1982). "Fatigue reliability, I - IV." J. Struct. Div. ASCE 108(1):1 - 88 .

Connolly, M.P. (1995). "Reliability based assessment of inspection requirements." Materials Evaluation 10: pp. $1191-1197$.

Czajkowski, C.J. (1990). "Survey of boric acid corrosion of carbon steel components in nuclear plants." NUREG/CR-5576, U.S. Nuclear Regulatory Commission, Washington, DC.

Davidson, J.R. (1973). "Reliability and structural integrity." 10th Anniversary Meeting of the Soc. of Engrg. Sci., Society of Engineering Science.

Dekraker, A., Tichler, S.W. and Vrouwenvelder, A. (1982). "Safety, reliability and service life of structures." HERON 27(1):Delft, The Netherlands.

Ditlevsen, O. (1986). "Random fatigue crack growth - a first passage problem." Engrg. Fract. Mech. 23(2467477. 
Doctor, S. R., et al (1993). "Nondestructive examination (NDE) reliability for inservice inspection of light water reactors." NUREG/CR-4469 (15 Volumes), Washington, DC.

Dolinski, K. (1992). "Stochastic modeling and statistical verification of crack growth under constant amplitude loading." Engrg. Fract, Mech, 43(2) 195-216.

Dowling, N.E. (1993). Mechanical behavior of materials. Prentice-Hall, Englewood Cliffs, NJ.

Ellingwood, B. (1976). "Probabilistic assessment of low-cycle fatigue behavior of structural welds." I. Pressure Vessel Tech. ASME, 98(1):26-32.

Ellingwood, B. and Hwang, H.(1985). "Probabilistic descriptions of resistance of safety-related structures in nuclear plants." Nuc, Engrg, and Des. 88(1):169-178.

Ellingwood, B. (1990). "Validation studies of seismic PRAs." Nuc. Engrg, and Des. 123(2):189-196.

Ellingwood, B. and Mori, Y. (1992). "Condition assessment and reliability-based life prediction of concrete structures in nuclear plants." Report ORNL/NRC/LTR-92/4, Oak Ridge National Laboratory, Oak Ridge, TN, $147 \mathrm{pp}$.

Ellingwood, B. (1992). "Probabilistic risk assessment." in Engineering Safety (D. Blockley, ed.) McGraw-Hill Book Company (UK) Ltd., pp. 89-116.

Ellingwood, B. and Mori, Y. (1993). "Probabilistic methods for condition assessment and life prediction of concrete structures in nuclear power plants." Nuc. Engrg, and Des. 142:155-166.

Ellingwood, B. (1983). "Probability based safety checking of nuclear plant structures." NUREG/CR-3628, U.S. Nuclear Regulatory Commission, Washington, DC.

Ellingwood, B. (1994). "Validation of seismic probabilistic risk assessments of nuclear power plants." Report NUREG/GR-0008, Washington, DC, 130 pp.

Ellingwood, B. (1996). "Reliability-based condition assessment and LRFD for existing structures." Struct. Safety 18(2):27-45.

Engelund, S. and Rackwitz, R. (1993). "A benchmark study on importance sampling techniques in structural reliability." Struct. Safety 12(4):255-276.

Fujita, M., Schall, G. and Rackwitz, R. (1989). "Adaptive reliability-based inspection strategies for structures subjected to fatigue." Structural Safety and Reliability (ICOSSAR'89), Vol. II, ASCE, New York, pp. 16191626.

Gabrielli, C., et al (1990). "A review of the probabilistic aspects of localized corrosion." Corrosion Science 46(4):266-278.

Galambos, T.V. and Ravindra, M.K. (1978). "Properties of steel for use in LRFD." I. Struct. Div. ASCE 104(9):1459-1468.

Ghorbanpoor, A. (1994). "An assessment of the current acoustic emission evaluation of steel bridges", Structural Materials Technology, (Scancella et al, ed.), Atlantic City, New Jersey, pp. 114-118. 
Hall, W.B. (1988). "Reliability of service-proven structures." I. Struct. Div, ASCE 114(3):608-623.

Heasler, P.G., Taylor, T.T., Spanner, J.C. et al. (1990). "Ultrasonic Inspection Reliability for Intergranular Stress Corrosion Cracks.: NUREG/CR-4908, Washington, DC.

Heasler, P.G., Taylor, T.T. and Doctor, S.R. (1993). "Statistically Based Reevaluation of PISC-II Round Robin Test Data," NUREG/CR-5410, Washington, DC.

Holman, G.S. (1989). "Application of reliability techniques to prioritize BWR recirculation loop welds for inservice inspection." NUREG/CR-5486, Washington, DC.

Hwang, H., et al (1983). "A consensus estimation study of nuclear power plant structural loads." NUREG/CR3315, U.S. Nuclear Regulatory Commission, Washington, DC.

Hwang, H., Ellingwood, B., Shinozuka, M. and Reich, M. (1987). "Probability-based design criteria for nuclear plant structures." J. of Struct. Engrg. ASCE 113(5):925-942.

Ihara, C. and Misawa, T. (1991). "Stochastic models related to fatigue damage of materials". J. of Energy Resources Tech., 113(4), pp 215-21.

Ishikawa, H., et al (1993). "Reliability assessment of structures based on probabilistic fracture mechanics." Probabilistic Engrg. Mech. 8(1):43-56.

Jaske, C. E. (1987). "Benefits of remaining life assessment." Chemical Engrg. Progress:37-46.

Jiles, D.C., Biner, S.B., Govindaraju, M.R. and Chen, Z.J. (1994). "Applications of a new magnetic monitoring technique to in situ evaluation of fatigue damage in ferrous components." NUREG/GR-0013, Washington, DC

Joshi, N.R. (1994). "Statistical analysis of UT corrosion data from floor plates of a crude oil aboveground storage tank." Materials Evaluation, vol 52, pp 846-9.

Kachanov, L.M. (1986). Introduction to continuum damage mechanics. Martinus Nijhoff, Dordrecht, the Netherlands.

Kameda, H.l and Koike, T. (1975). "Reliability theory of deteriorating structures." J. Struct. Div. ASCE 101(1):295-309.

Karamchandani, A., Dalane, J.I. and Bjerager, P. (1992). "System reliability approach to fatigue of structures." J. Struct. Engrg. ASCE 118(3):684-700.

Kayser, J.R. and Nowak, A.S. (1989). "Reliability of corroded steel girder bridges." Struct. Safety 6(1):53-63.

Keating, and Fisher, J.W. (1986). "Evaluation of fatigue tests and design criteria on welded details." NCHRP Report 286, Transportation Research Board, Washington, DC.

Keisler, J., Chopra, O.K. and Shack, W.J. (1994). "Statistical analysis of fatigue strain-life data for carbon and low-alloy steels." Report NUREG/CR-6237, Washington, DC, 36 pp.

Kennedy, E. L., Foulds, J.R. and Basin, S.L. (1991). "Nuclear reactor pressure vessel flaw distribution development Phase II - methodology and application." Report SAND91-7073, Sandia National Laboratories, Albuquerque, NM. 
Kennedy, R.P. and Ravindra, M.K. (1984). "Seismic fragilities for nuclear power plant studies." Nuc. Engrg. and Des. 79(1):47-68.

Kishi, T. (1988). "Nondestructive Evaluation of Civil Structures in Japan." Proceedings of the International Workshop on Nondestructive Evaluation for Performance of Civil Structures, (Agbabian, M.S. and Masri, S.F., ed.) California, pp 63-79, 1988.

Kobayashi, T., Shockey, D.A. and Jones, R.L. (1991). "Deriving SCC initiation times and growth rates from posttest fractographic analysis." Corrosion Science 47(7):528-535.

Komp, M.E. (1987). "Atmospheric corrosion ratings of weathering steels - calculation and significance." Materials Performance 26(7):42-44.

Kondo, Y. (1989). "Prediction of fatigue crack initiation life based on pit growth." Corrosion Science 45(1):711.

Kung, C.J. and Wirsching, P.H. (1993). "Fatigue/fracture reliability and maintainability process for structural systems." in Computational Stochastic Mechanics (Cheng and Yang, eds.), Elsevier, London, pp. 369 - 393.

LRFD specification for structural steel buildings (1993). American Institute of Steel Construction, Chicago, IL.

Lemaitre, Jean (1992). A course on damage mechanics. Springer-Verlag, Berlin, Germany.

Lewis, H.W. (1985). "Medians and means in probabilistic risk assessment." Nuc. Science and Engrg. 91:220222.

Lin, Y.K. and Yang, J.N! (1983). "On statistical moments of fatigue crack propagation", Engrg. Frac. Mech. Mechaqnics, vol. 18, no. 2, pp. 243-256, 1983.

Lutes, L.D., Corazao, J., Hu, S.J., and Zimmerman, J (1984). "Stochastic fatigue damage accumulation." I. Struct. Engrg. ASCE 110(11): 2585-2601.

Madsen, H.O. (1982). "Deterministic and probabilistic models for damage accumulation due to time varying loads." Dialog 5-82, Danish Engineering Academy, Lyngby.

Madsen, H.O. (1987). "Model updating in reliability theory." Reliability and Risk Analysis in Civil Engineering (ICASP 5. 1987), University of Waterloo, pp. 564-577.

Madsen, H.O., Sorensen, J.D. and Olesen, R. (1989). "Optimal inspection planning for fatigue damage of offshore structures." Structural Safety and Reliability (ICOSSAR'89), Vol. III, ASCE, New York, pp. 20992106.

Marsh, G.P., et al (1985). "Evaluation of localised corrosion of carbon steel overpacks for nuclear waste disposal in granite environments." Symposium on Scientific Basis for Nuclear Waste Management IX," Materials Research Society Symposium Proceedings Vol. 50, Pittsburgh, PA. pp. 421-428.

Meister, D. (1982). "Human factors in reliability." Reliability Handbook (Ireson, ed.,) McGraw-Hill, NY. Melchers, R.E. (1987). Structural reliability - analysis and prediction. Ellis Horwood, Chichester, UK. 
Melchers, R.G. (1990). "Search-based importance sampling." Struct. Safety 9(2):117-128.

Mola, E.E., Mellein, B., Rodriguez, E.M., Vicente, J.L., Salvarezza, R.C. and Arvia, A.J. (1990). "Stochastic approach for pitting corrosion modeling I - The case of quasi-hemispherical pits." J. Electrochem. Soc. 137(5): 1384-1390.

Mori,Y. and Ellingwood, B. (1993). "Reliability-based service life assessment of aging concrete structures." J. Struct. Engrg. ASCE 119(5):1600-1621.

Mori, Y and Ellingwood, B. (1993). "Methodology for reliability-based condition assessment - Application to concrete structures in nuclear plants." Report NUREG/CR-6052, ORNL/sub/93-SD684, U.S. Nuclear Regulatory Commission, Washington, DC, 145 pp.

Mori, Y. and Ellingwood, B. (1994a). "Maintaining reliability of concrete structures I: Role of inspection/repair." J. Struct. Engrg. ASCE 120(3):824-845.

Mori, Y. and Ellingwood, B. (1994b). "Maintaining reliability of concrete structures II: Optimum inspection and repair." J.Struct. Engrg. ASCE 120(3):846-867.

Moses, F. (1990). "New directions and research needs in system reliability research." Struct. Safety 7(2):93100.

Murakami, S. and Mizuno, M. (1991). "Elaborated constitutive equations for structural analysis for creep, swelling and damage under irradiation." Nuclear Technology 95:219-227 (August 1991)

Natalie, C.A. (1987). "Evaluation of uniform corrosion." ASM Handbook, Volume 13 - Corrosion, ASM International, pp. 229-230.

Naus, D.J. (1986). "Concrete component aging and its significance relative to life extension of nuclear po wer plants." NUREG/CR-4652 (ORNL/TM-10059), Oak Ridge National Laboratory, Oak Ridge, TN.

Naus, D.J., Oland, C.B., Ellingwood, B., Mori, Y. and Arndt, E.G. (1993). "An overview of the ORNL/NRC program to address aging of concrete structures in nuclear power plants." Nuc. Engrg. and Des. 142:327-339.

Naus, D.J., Oland, C.B. and Ellingwood, B. (1996). "Final report on aging of nuclear power plant reinforced concrete structures." NUREG/CR-6424, U.S. Nuclear Regulatory Commission, Washington, DC.

Nienstedt, J., Tsurui, A., Tanaka, H. and Schueller, G.I. (1990). "Time-variant structural reliability analysis using bivariate diffusive crack growth models." Int. J. Fatigue 12(2):83-89.

Okrent, D. (1987). "The safety goals of the U.S. Nuclear Regulatory Commission." Science 236(4799):233364.

Oland, C.B. and Naus, D.J. (1996). "Degradation assessment methodology for application to steel containments and liners of reinforced concrete structures in nuclear power plants." Report ORNL/NRC/LTR95/29, Oak Ridge National Laboratory, Oak Ridge, TN.

Ortiz, K. and Kiremidjian, A.S. (1986). "Time series analysis of fatigue crack growth rate data." Engrg. Fract. Mech., vol. 24, no. 5, pp. 657-675. 
Oswald, G.F. and Schueller, G.I. (1984). "Reliability of deteriorating structures." Engrg. Fract. Mech. 20(3):479-488.

Packman, P.F., Pearson, H.S., Owens, J.S. and Young, G. (1969). "Definition of Fatigue Cracks through Nondestructive Testing." Joumal of Materials, Vol. 4, 1969, pp. 666-700.

Pate-Cornell, E. (1994). "Numerical safety goals for engineering risk management." Risk Analysis, Univ. of Michigan, Ann Arbor, MI, pp. 175-191.

Pearce, T.H. and Wen, Y.K. (1985). "Stochastic combinations of load effects." ․ Struct. Engrg. ASCE 110(7):1613-1629.

Porter, F.M., Naish, C.C. and Sharland, S.M. (1994). "A probabilistic approach to assessing radioactive waste container lifetimes." Proc, ANS Conf. on High-level Waste, Las Vegas, NV, May.

Provan, J.W. (1987). "Probabilistic approaches to the material-related reliability of fracture-sensitive stuctures." in Probabilistic Fracture Mechanics and Reliablity, (Dordrecht; Boston) Martinus Nijhoff Publishers, The Netherlands.

Provan, J.W. and Rodriguez, E.S. (1989). "Part I: Development of a Markovian description of pitting corrosion." Corrosion 45(3): 178-192.

Rackwitz, R. (1985). "Reliability of systems under renewal pulse loading." I. Engrg. Mech. ASCE 111(9):1175-1184.

Rahman S. and Grigoriu, M. (1993). "Markov model for seismic reliability analysis of degrading structures." J. Struct. Engrg. ASĊE 119(6):1844-1865.

Rajashekhar, M.R. and Ellingwood, B.R. (1995). "Reliability of reinforced-concrete cylindrical shells." J. Struct. Engrg. ASCE 121(2):336-347.

Rocha, M.M., Schueller, G.I. and Okamura, H. (1993). "The fitting of one- and two-dimensional fatigue crack growth laws." Engrg. Fract. Mech., vol 44, no 3, pp 473-480, 1993.

Rodrigues, E.S. and Provan, J.M. (1989). "Part II: development of a general failure control system for estimating the reliability of deteriorating structures." Corrosion 45(3):193-206.

Rogers, W.F. (1990). "Statistical prediction of corrosion failures." Materials Performance 29(6):40-44.

Rubenstein, R.Y. (1981). Simulation and the Monte Carlo Method. John Wiley, New York.

Rummel, W.D., Hardy, G.L. and Cooper, T.D. (1989). Metals Handbook, Ninth Edition, Vol. 17. Nondestructive Evaluation and Quality Control. ASM International, Materials Park, Ohio, 674-688.

Sarkani, S., Buresli, N. and Michaelov, G. (1994). "Fatigue damage accumulation under stochastic stress histories." Proc. Structures Congress XII, Vol, 1, American Society of Civil Engineers, New York, NY, pp. 833-838.

Scarf, P.A. and Laycock, P.J. (1994). "Application of extreme value theory in corrosion engineering", Journal of Research of the National Institute of Standards and Technology, vol 99, no 4, pp 313-20. 
Schueller, G.I. and Stix, R. (1987). "A critical appraisal of methods to determine failure probabilities." Struct. Safety 4(4):293-310.

Shah, V.N. and MacDonald, P.E., eds. (1989). "Residual life assessment of major light water reactor components - overview." Report NUREG/CR-4731, 2 Vols., Washington, DC.

Shah,V.N., Smith, S.K. and Sinha, U.P. (1994). "Insights for aging management of light water reactor components - steel containments." NUREG/CR-5314, Vol. 5, U.S., Washington, DC.

Sharland, S.M., et al (1991). "The assessment of localised corrosion of carbon and stainless steel containers for intermediate- and low-level radioactive waste under repository conditions." ANS Conf, on Nuclear Waste Packaging, Las Vegas, NV, Sept.

Shibata, T. (1994). "Application of extreme value statistics to corrosion." J. of Research of the N.I.S.T. vol $99, \mathrm{n} \bullet 4, \mathrm{pp} 327-36$.

Shinozuka, M. (1983). "Basic analysis of structural safety." I. Struct. Engrg. ASCE 109(3):721-740.

Siemes, A.J.M., Vrouwenvelder, A. and van den Beukel, A. (1985). "Durability of buildings: a reliability analysis." $\underline{\text { HERON } 30(3): 1-48 . ~}$

Sommer, A., Nowak, A.S. and Thoft-Christensen, P. (1993). "Probabilisty-based bridge inspection strategy." J. Struct. Engrg. ASCE 119(12):3520-3536.

Sorensen, J.D., Kroon, I.B. and Faber, M.H. (1994). "Optimal reliability-based code calibration." Structural Safety 15(3):197-208.

Spencer, B.F. Jr. and Tang, J. (1988). "Markov process model for fatigue crack growth." J. of Engrg. Mech.. ASCE 114,(12):2134-57.

Sprowls, D.O. (1987). "Evaluation of pitting corrosion." ASM Handbook. Volume 13 - Corrosion, ASM International, pp. 231-233.

Staat, M. (1993). "Sensitivity of and influences on the reliability of an HTR-module primary circuit pressure boundary." Trans., 12th Int Conf. on Struct. Mech, in Reactor Tech., Vol. M, Elsevier, Amsterdam, the Netherlands, pp. 147 - 152.

"Standard Review Plan, Rev.1 (1981)." NUREG-0800, U.S. Nuclear Regulatory Commission, Washington, DC.

Strelec, H. (1993). "A model for accelerated life testing." Struct. Safety 12(2):129-136.

Structural Alloys Handbook, Volume 3 (1989). Metals and Ceramics Information Center, Battelle, Columbus, $\mathrm{OH}$.

Strutt, J.E., Nichells, J.R. and Barbier, M.E. (1985). "The prediction of corrosion by statistical analysis of corrosion profiles." Corrosion Sci. 25(5):

Topper, T.H., et al (1969). "Neuber's rule applied to fatigue of notched specimens." I. Materials JMLSA, 4(1):200-209. 
Torng, T.Y. and Wirsching, P.H. (1991). "Fatigue and fracture reliability and maintainability process." I. Struct, Engrg. ASCE 117(12):3804-3822.

Tsai, C.-H. and Wu, W.-F. (1993). "Application of probabilistic fracture mechanics to risk assessment of pressure vessels." Trans., 12th Int Conf. on Struct. Mech. in Reactor Tech., Vol. M, Elsevier, Amsterdam, the Netherlands, pp. $135-140$.

Vo, T.V., et al (1994a). "Feasibility of developing risk-based rankings of pressure boundary systems for inservice inspection." NUREG/CR-6151, Washington, DC.

Vo, T.V., et al (1994b). "A pilot application of risk-based methods to establish in-service inspection priorities for nuclear components at Surry Unit 1 nuclear power station." NUREG/CR-6181, Washington, DC.

Vora, J.P., et al (1991). "Nuclear plant aging research (NPAR) program plan." NUREG-1144, Rev. 2, U.S. Nuclear Regulatory Commission, Washington, DC.

Walther, H.P. (1992). "Evaluation of behavior and radial shear strength of a reinforced concrete containment structure." NUREG/CR-5674, Washington, DC.

Ware, A.G., Morton, D.K. and Nitzel, M.E. "Application of NUREG/CR-5999 interim fatigue curves to selected nuclear power plant components." NUREG/CR-6260, Washington, DC.

Williams, D.E., Westcott, C. and Fleishmann, M. (1985). "Stochastic models of pitting corrosion of stainless steels." J. Electrochem. Soc. 132(8): 1796-1804.

Wirsching, P. and Light, M. (1980). "Fatigue under wide band random stresses." J. Struct. Div. ASCE 106(7):1593-1607.

Wunderlich, W.O. (1991). "Probabilistic methods for maintenance." J. Engrg. Mech. ASCE 117(9):20652078.

Yao, J.T.P. et al (1986). "Stochastic Fatigue, Fracture and Damage Analysis." Struct. Safety 3(4):231 - 267.

Yeh, J.C. , Enneking, J.A. and Tsai, C.L. (1994). "Study of acoustic emission characteristics for fracture assessment of structural weldment." Review of Progress in Quantitative Nondestrcutive Evaluation, Vol. 13 (Thompson et al, ed.). Plenum Press, New York, pp.477-483,1994.

Yoshimura, S., Zhang, M.-Y., and Yagawa, G. (1993). "Life extension simulation of aged RPV material using probabilistic fracture mechanics analysis on massively parallel computer." Trans. 12th Int Conf. on Struct. Mech, in Reactor Tech. Vol.,M, Elsevier, Amsterdam, the Netherlands, pp. 165 - 176.

Zhao, H. (1993). "An improved probabilistic model of fatigue crack growth." Engrg. Fract. Mech. 46(5): 773780 .

Zhu, W.Q., Lin, Y.K. and Lei, Y. (1992). "On fatigue crack growth under random loading." Engrg. Fract. Mech., 43(1), pp. 1-12. 

NUREG/CR-5442

ORNL/TM-13244

Dist. Category RD, R9

\section{INTERNAL DISTRIBUTION}

1. W. G. Craddick

2. R. G. Gilliland

3-12. D. J. Naus

13-20. C. B. Oland

21. C. E. Pugh

22. J. O. Stiegler
23. ORNL Patent Section

24. Central Research Library

25. Document Reference Section

26-27. Laboratory Records

28. Laboratory Records, ORNL-RC

\section{EXTERNAL DISTRIBUTION}

29. H. G. Ashar, Division of Engineering, Office of Nuclear Reactor Regulation, Nuclear Regulatory Commission, Washington, DC 20555-0001

30. G. Bagchi, Division of Engineering, Office of Nuclear Reactor Regulation, Nuclear Regulatory Commission, Washington, DC 20555-0001

31. J. Carey, Electric Power Research Institute, 3412 Hillview Avenue, Palo Alto, CA 94304

32. K. N. Chao, Consumers Power Co., 1945 West Parnell Road, Jackson, MI 49201

33. J. R. Clifton, National Institute of Standards of Technology, Building 226, Room B-348, Gaithersburg, MD 20899

34. Commander and Director, USAE Waterways Experiment Station, ATTN: CEWESIM-MI-R, Alfreda S. Clark, CD Dept. 1 \#1072, 3909 Halls Ferry Road, Vicksburg, MS 39180-6199

35. J. F. Costello, Division of Engineering Technology, Office of Nuclear Regulatory Research, Nuclear Regulatory Commission, Washington, DC 20555-0001

36. K. Cozens, NUMARC, 1776 I Street, NW, Washington, DC 20006

37. C. Cragg, Ontario Hydro, 700 University Avenue, Toronto, Ontario MSG 1X6, Canada

38. J. W. Craig, Division of Engineering Technology, Office of Nuclear Regulatory Research, Nuclear Regulatory Commission, Washington, DC 20555-0001

39. D. R. Eggett, Commonwealth Edison, 1400 Opus Place, Suite 500, Downers Grove, IL 60515

40-50. B. Ellingwood, Professor of Civil Engineering, The Johns Hopkins University, 3400 North Charles St., Baltimore, MD 21218-2699

51. G. Fordyce, HM Principal Inspector, Nuclear Installations Inspectorate, St. Peters House, Balliol Road, Bootle, Merseyside L20 3LZ, England

52. G. Frohnsdorff, National Institute of Standards of Technology, Building 226, Room B-368, Gaithersburg, MD 20899

53. W. L. Gamble, Professor of Civil Engineering, University of Illinois, 3122 Newmark Civil Engineering Laboratory, 205 N. Mathews, Urbana, IL 61801

54. H. Graves, III, Division of Engineering Technology, Office of Nuclear Regulatory Research, Nuclear Regulatory Commission, Washington, DC 20555-0001

55. D. C. Jeng, Division of Engineering, Office of Nuclear Reactor Regulation, Nuclear Regulatory Commission, Washington, DC 20555-0001 
56. R. Judge, AEA Technology, Bldg. 329, Harwell, Didcot, Oxford, OX11 ORA, United Kingdom

57. D. Kluge, Swiss Federal Nuclear Safety Inspectorate (HSK), CH-5303, Würenlingen, Switzerland

58. P. Krauss, Wiss, Janney, Elstner Associates, Inc., 330 Pfingsten Road, Northbrook, IL 60062-2095

59. P-T. Kuo, Division of Reactor Program Management, Office of Nuclear Reactor Regulation, Nuclear Regulatory Commission, Washington, DC 20555-0001

60. T. C. Liu, Chief, Materials Engineering Section, Headquarters, U.S. Army Corps of Engineers, 20 Massachusetts Ave., NW, HQUSACE (CECW-EG), Washington, DC 20314

61. M. E. Mayfield, Division of Engineering Technology, Office of Nuclear Regulatory Research, Nuclear Regulatory Commission, Washington, DC 20555-0001

62. W. McCleese, U.S. Army Corps of Engineers, Waterways Experiment Station, (CEWES-SC-A), 3909 Halls Ferry Road, Vicksburg, MS 39180-6199

63. A. Murphy, Division of Engineering Technology, Office of Nuclear Regulatory Research, Nuclear Regulatory Commission, Washington, DC 20555-0001

64-68. W. Norris, Division of Engineering Technology, Office of Nuclear Regulatory Research, Nuclear Regulatory Commission, Washington, DC 20555-0001

69. J. Philip, Division of Regulatory Applications, Office of Nuclear Regulatory Research, Nuclear Regulatory Commission, Washington, DC 20555-0001

70. M. K. Ravindra, EQE International, 44 Montgomery Street, Suite 3200, San Francisco, CA 94104-4805

71. J. W. Reed, Jack R. Benjamin and Assoc., 444 Castro St., Suite 501, Mountain View, CA 94041

72. R. F. Sammataro, Proto-Power Corporation, 591 Poquonnock Road, Groton, CT 06340

73. L. C. Shao, Division of Engineering Technology, Office of Nuclear Regulatory Research, Nuclear Regulatory Commission, Washington, DC 20555-0001

74. R. E. Shewmaker, Office of Nuclear Materials Safety \& Safeguards, Low-Level Waste Management Branch, Nuclear Regulatory Commission, Washington, DC 20555-0001

75. M. Vagins, Division of Engineering Technology, Office of Nuclear Regulatory Research, Nuclear Regulatory Commission, Washington, DC 20555-0001

76. J. P. Vora, Division of Engineering Technology, Office of Nuclear Regulatory Research, Nuclear Regulatory Commission, Washington, DC 20555-0001

77. R. Winkel, Westinghouse Hanford Company, P.O. Box 1970-HF-57, Richland, WA 99352

78. P. Zwicky, Basler \& Hofmann, Forchstrasse 395, CH-8029 Zurich, Switzerland 


\begin{tabular}{|c|c|}
\hline $\begin{array}{l}\text { U.S. NUGLEAR REGULATORY COMMISSION } \\
\text { BIBLIOGRAPHIC DATA SHEET } \\
\text { (See instructions on the reverse) }\end{array}$ & $\begin{array}{l}\text { 1. REPORT NUMBER } \\
\text { (Assioned by NRC. Add Vol., Supp., Rev., } \\
\text { and Addendum Numbers, if any.) } \\
\text { NUREG/CR-5442 }\end{array}$ \\
\hline \multicolumn{2}{|l|}{ 2. TITLE AND SUBTITLE } \\
\hline \multirow{3}{*}{$\begin{array}{l}\text { Reliability-Based Condition Assessment of Steel } \\
\text { Containments and Liners }\end{array}$} & DATE REPORT PUBLISHED \\
\hline & \begin{tabular}{|r|r|} 
MONTH & YEAR \\
November & 1996 \\
\end{tabular} \\
\hline & $\begin{array}{l}\text { 4. FIN OR GRANT NUMBER } \\
\text { J6043 }\end{array}$ \\
\hline \multirow{2}{*}{$\begin{array}{l}\text { 5. AUTHOR(S) } \\
\text { B. R. Ellingwood, B. Bhattacharya, and R-H Zheng } \\
\text { (The Johns Hopkins University) }\end{array}$} & $\begin{aligned} \text { 6. TYPE OF REPORT } \\
\text { Technical }\end{aligned}$ \\
\hline & 7. PERIOD COVERED (IInclusive Dates) \\
\hline
\end{tabular}

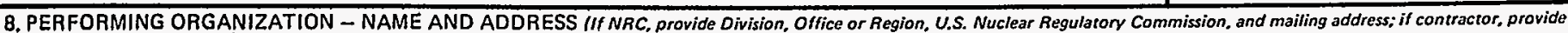
name and mailing address.

\section{Contractor}

Oak Ridge National Laboratory

P.0. Box 2009, Bldg., 9204-1

Oak Ridge, Tennessee 37831-8056
Subcontractor

The Johns Hopkins University

Dept. of Civil Engineering

3400 N. Charles St 2699

9. SPONSORING ORGANIZATION - NAME AND ADDRESS IIINRG, type "Same as above": if contractor, provide NRC Division, Office or Region, U.S. Nuclear Regulatory Commission, and malling address.

Division of Engineering Technology

Office of Nuclear Regulatory Research

U.S. Nuclear Regulatory Commission

Washington, D.C. 20555-0001

10. SUPPLEMENTARY NOTES

W.E. Norris, NRC Project Manager

11. ABSTRACT (200 words or /ess)

The evaluation of steel containments and liners for continued service must provide assurance that they are able to withstand future extreme loads during the service period with a level of reliability that is sufficient for public safety. This research demonstrates the feasibility of using reliability analysis as a tool for performing condition assessments and service life predictions of steel containment and liners. Mathematical models that describe time-dependent charges in steel due to aggressive environmental factors are identified, and statistical data supporting the use of these models in time-dependent reliability analysis are summarized. The analysis of steel containment fragility is described, and simple illustrations of the impact on reliability of structural degradation are provided. The role of nondestructive evaluation in time-dependent reliability analysis, both in terms of defect detection and sizing, is examined. A Markov model provides a tool for accounting for time-dependent changes in damage condition of a structural component or system.

12. KEY WORDS/DESCR!PTORS (List words or phrases that will assist researchers in locating the report.)

Aging

Containment (Stee1)

Corrosion

Fatigue

Fracture Mechanics

Nondestructive Evaluation

Probability Theory

Reliability
Risk

Statistics

Structural Engineering

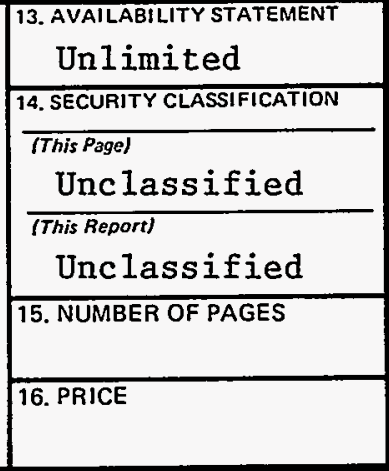




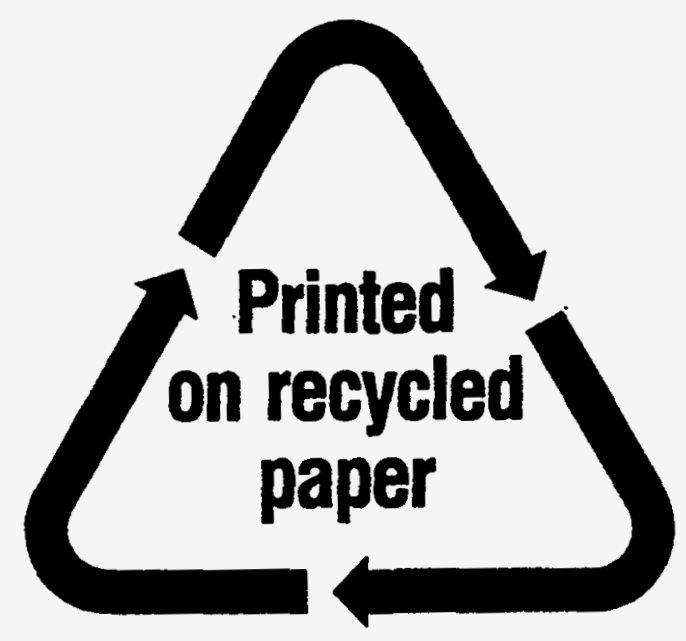

Federal Recycling Program 